Aus dem Institut für Allgemeinmedizin

(Prof. Dr. med. E. Hummers)

der Medizinischen Fakultät der Universität Göttingen

\title{
Wahrnehmungen und Bedürfnisse von Hausärzten hinsichtlich ihrer Tätigkeit im Pflegeheim
}

\author{
INAUGURAL - DISSERTATION \\ zur Erlangung des Doktorgrades \\ der Medizinischen Fakultät der \\ Georg-August-Universität zu Göttingen
}

vorgelegt von

Franziska Blank

aus

Bad Nauheim

Göttingen 2018 
Dekan:

Referent/in:

Ko-Referent/in:
Prof. Dr. rer. nat. H. K. Kroemer

Prof. Dr. E. Hummers

Prof. Dr. M. Schweda

Datum der mündlichen Prüfung: 20.02.2020 
Hiermit erkläre ich, die Dissertation mit dem Titel "Wahrnehmungen und Bedürfnisse von Hausärzten hinsichtlich ihrer Tätigkeit im Pflegeheim" eigenständig angefertigt und keine anderen als die von mir angegebenen Quellen und Hilfsmittel verwendet zu haben.

Göttingen, den 


\section{Inhaltsverzeichnis}

1. EINLEITUNG 1

2. STAND DER FORSCHUNG 4

2.1. Allgemeine Situation in Deutschland 4

2.2. ARZTPERSPEKTIVE In DeUtSChLAND

2.3. ARZtPerspektive AUf die Arbeit im Heim in DeUtschland 17

$\begin{array}{lr}\text { 2.4. INTERnationale Perspektive } & 19\end{array}$

3. $\quad$ FRAGESTELLUNG UND ZIEL 28

4. MATERIAL UND METHODEN

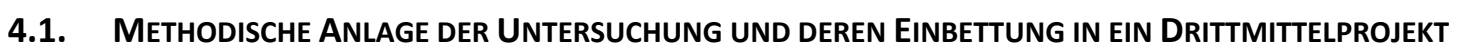

4.2. ReKRUtierUNG DER TEILNEHMENDEN HAUSÄRZTE UND EINWILLIGUNGSGESPRÄCH 32

4.3. INTERVIEWLEITFADEN

4.4. DURCHFÜHRUNG DER UNTERSUCHUNG 35

4.5. Datenanalyse 36

4.6. Datenschutz Und Genehmigung DURCh die EthiKKommission 41

5. ERGEBNISSE $\quad 42$

5.1. Beschreibung Der StichPROBE 42

5.2. ERGEBNISSE DER INTERVIEWAUSWERTUNG

5.2.1. Wahrnehmungen und Bedürfnisse der Hausärzte bezüglich ihrer eigenen Arbeitssituation - Wünsche hinsichtlich einer Lösungsfindung

5.2.1.1. Wahrnehmung der externen Rahmenbedingungen und Bedürfnisse zu den externen

Rahmenbedingungen $\quad 45$

5.2.1.2. Wahrnehmung der heiminternen Rahmenbedingungen und Bedürfnisse zu den heiminternen Rahmenbedingungen $\quad 52$

5.2.1.3. Sicht der Hausärzte auf das Thema „Heimarzt" 63

5.2.1.4. Allgemeine Lösungsvorschläge hinsichtlich der Arbeitssituation 66 
5.2.2. Wahrnehmungen und Bedürfnisse der Hausärzte hinsichtlich der Versorgung und Betreuung der Bewohner $\quad 68$

5.2.2.1. Bewohnersicherheit in der medizinischen Versorgung 68

5.2.2.2. Aufgaben der Pflegekräfte aus Hausarztsicht 70

5.2.2.3. Weitere allgemeine Vorstellungen und Wünsche zur Bewohnerversorgung 77

5.2.3. Wahrnehmungen und Bedürfnisse bezüglich des Tätigkeitsbereichs Pflegeheim.

Wahrnehmung des Arbeitsumfelds Pflegeheim - Das zentrale Phänomen der Zerrissenheit 79

5.2.3.1. Ursächliche Bedingungen 81

5.2.3.2. Intervenierende Bedingungen 82

$\begin{array}{llr}\text { 5.2.3.3. Strategien } & 94\end{array}$

$\begin{array}{lll}\text { 5.2.3.4. Konsequenzen } & 106\end{array}$

6. DISKUSSION 116

6.1. ÜBERSICHT ÜBER DIE WICHTIGSTEN ERGEBNISSE 116

$\begin{array}{ll}\text { 6.2. StÄRKEN UND SCHWÄCHEN DER ARBEIT } & 117\end{array}$

6.3. EINORDNUNG INNERHALB DER THEMENBEZOGENEN LITERATUR 118

$\begin{array}{lll}\text { 6.4. SCHLUSSFOLGERUNG } & 128\end{array}$

7. ZUSAMMENFASSUNG $\quad 132$

$\begin{array}{ll}\text { 7.1. SUMMARY } & 135\end{array}$

\begin{tabular}{lr} 
8. & ANHANG \\
\hline
\end{tabular}

9.

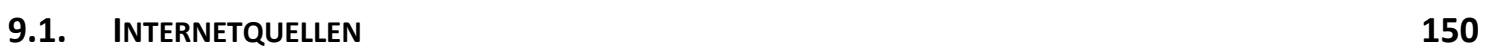




\section{Abbildungsverzeichnis}

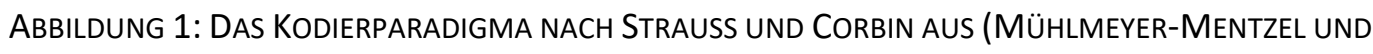
SCHÜRMANN 2011). Mit FREUNDLICHER GENEHMIGUNG DER AUTORINNEN.

ABBildung 2: ALtersVERTEILUNG DER INTERVIEWTEN HAUSÄRZTE

ABBildung 3: ANZAHL DER VERSORGTEN HEIMPATIENTEN PRO HAUSARZT

ABBILDUNG 4: ÜBERSICHT ZU DEN EXTERNEN RAHMENBEDINGUNGEN

ABBILDUNG 5: ÜBERSICHT ZU DEN HEIMINTERNEN RAHMENBEDINGUNGEN

ABBILDUNG 6: ÜBERSICHT ZUM THEMA „HEIMARZT“

ABBILDUNG 8: ÜBERSICHT ZU DEN ALLGEMEINEN VORSTELLUNGEN UND WÜNSCHEN ZUR BEWOHNERVERSORGUNG

ABBILDUNG 9: ÜBERSICHT ZU DEN URSÄCHLICHEN UND INTERVENIERENDEN BEDINGUNGEN, STRATEGIEN, KONSEQUENZEN

ABBILDUNG 10: URSÄCHLICHE BEDINGUNGEN 


\section{Tabellenverzeichnis}

TABELLE 1: HAUSÄRZTLICHE VERGÜTUNG

ZAHLEN AUS (KASSENÄRZTLICHE BUNDESVEREINIGUNG 2018)

TABelle 2: Wegpauschalen Niedersachsen

Zahlen aus (KASSENÄrzTLICHe Vereinigung NiederSACHSEN 2018)

7

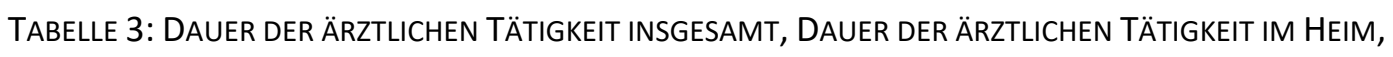
DAUER DER TÄTIGKEIT IM HEIM PRO WOCHE 


\section{Einleitung}

Demografischer Wandel - ein Begriff, der sich mit immer dringlicher werdender Präsenz durch Gesellschaft, Medien und Politik zieht. Dabei wird dieser rein deskriptive Ausdruck gleichgesetzt mit einer Entwicklung der deutschen Bevölkerung, in der es immer mehr ältere Menschen gibt, die zudem die Anzahl der Jüngeren überwiegen. Genau diese Entwicklung stellt Deutschland und andere entwickelte Länder vor allumfassende Herausforderungen. Besonders das Pflegewesen ist hierbei stark betroffen. Denn nicht nur das Alter unserer Bevölkerung nimmt zu, auch die Anzahl derer, die pflegebedürftig und somit auf (professionelle) Hilfe angewiesen sind, steigt. Die Bundesregierung erklärte 2012: „In wenigen Jahrzehnten wird die Zahl der pflegebedürftigen Personen auf über vier Millionen Menschen steigen“ (Deutscher Bundestag 2012, S.1). Es stellt sich die Frage, wer diese Versorgung leisten kann und unter welchen Bedingungen diejenigen dazu bereit sind.

Der Großteil der Pflegebedürftigen wird nach wie vor im häuslichen Rahmen gepflegt, ggf. mit Unterstützung durch ambulante Pflegedienste. Nichtsdestoweniger steigt auch der Bedarf an stationärer pflegerischer Versorgung: Laut Statistischem Bundesamt waren zu Ende 2015 insgesamt 783.416 Personen in vollstationären Pflegeeinrichtungen untergebracht (Statistisches Bundesamt 2017). Im Vergleich zu 2001 ist diese Anzahl um 32,4\% gestiegen (Statistisches Bundesamt 2017) und Experten sehen auch für die Zukunft einen deutlichen Aufwärtstrend (Bundesministerium für Gesundheit 2012). Diese Entwicklung bedeutet unter anderem: Die bestehenden Umstände in der stationären Versorgung von alten und pflegebedürftigen Menschen bedürfen einer Erneuerung und Anpassung an die demografische Entwicklung. 
Um die Versorgung von Pflegeheimbewohnern ${ }^{1}$ jedoch bestmöglich zu gewährleisten, ist es wichtig, die Interaktionspartner in den Fokus zu rücken. Neben den versorgenden Pflegekräften sollte ein besonderes Augenmerk auf die ärztliche Versorgung im Pflegeheim gerichtet werden. Die spezielle Bewohnerklientel im Pflegeheim, mit einer Häufung an multimorbiden und chronisch erkrankten Heimbewohnern (van den Bussche et al. 2009), stellt besondere Anforderungen an die versorgenden Ärzte.

Von politischer Seite wurde bereits erkannt, dass die Notwendigkeit einer Verbesserung der ärztlichen Versorgung im Pflegeheim besteht. Ziel des am 01. Januar 2013 in Kraft getretenen Pflege-Neuausrichtungs-Gesetzes (Bundesministerium für Gesundheit 2012) ist diesbezüglich, dass auf Antrag der Pflegeheime Kooperationsverträge mit entsprechend geeigneten Ärzten zu vermitteln sind. Die Pflegeheime ihrerseits werden dazu angehalten, Bericht über Art und Umfang der medizinischen Versorgung ihrer Bewohner zu erstatten. Dies soll zu einer sichergestellten und verbesserten Versorgung der Pflegeheimbewohner beitragen. Für die ärztliche Seite besteht der Anreiz, solche Verträge abzuschließen, in zusätzlichen finanziellen Boni. Zweck dieses Vergütungsanreizes soll nach dem postulierten Willen des Gesetzgebers sowohl bei dieser Gesetzesänderung als auch grundsätzlich beim Thema medizinische Versorgung stets die Verbesserung des Wohls der Patienten sein. Jedoch spielen sicherlich die finanziellen Ausgaben für dieses Vorhaben eine nicht so stark in den Vordergrund gestellte, aber wichtige Rolle. Fast gänzlich in den Hintergrund tritt dabei das Befinden der Ärzte. Bezüglich der hausärztlichen Versorgung im Pflegeheim ist es jedoch unerlässlich, auch die Perspektive der Hausärzte einzunehmen und deren Situation und Bedürfnisse zu beleuchten. Es ist fraglich, ob finanzielle Anreize ausreichen, um die Situation in der Pflegelandschaft Deutschlands für die derzeit behandelnden und zukünftigen Hausärzte zufriedenstellend zu gestalten. Vielmehr gilt es, die Einstellungen und die Wünsche von Hausärzten in Bezug auf ihre Arbeitssituation im Pflegeheim zu beleuchten

\footnotetext{
${ }^{1}$ In der vorliegenden Arbeit wird aus Gründen der besseren Lesbarkeit einheitlich die maskuline Form verwendet. Es wird sich jedoch auf alle Geschlechter bezogen.
} 
und zu klären, ob und wie die theoretischen Ansprüche praktisch umgesetzt werden können.

Jürgen Brüggemann, Fachgebietsleiter Qualitätsmanagement Pflege beim Medizinischen Dienst der Spitzenverbände der Krankenkassen erklärte: „Uns liegen keine genauen Daten zur Qualität der ärztlichen Versorgung in Heimen vor. Aber unsere Erfahrung zeigt, dass die Schnittstelle niedergelassener Arzt und Heim ein großes Problem ist" (Hibbeler 2007, S.3298). Diese bestehende Schnittstellenproblematik macht deutlich, dass die Notwendigkeit besteht, eine intensive Untersuchung der ärztlichen Sicht auf das Arbeitsumfeld Pflegeheim vorzunehmen. Durch ein besseres Verständnis der Arztperspektive können bessere Bedingungen für Ärzte in der Heimversorgung geschaffen werden, was schließlich zu einer verbesserten interprofessionellen Zusammenarbeit zwischen Ärzten und Pflegekräften und auch zu einer verbesserten medizinischen Versorgung der Pflegeheimbewohner führen kann.

Um die medizinische Versorgung der alternden Bevölkerung auch in Zukunft sicherstellen zu können, müssen demnach besonders die die hausärztliche Versorgung leistenden Ärzte bei Optimierungsvorhaben in diesem Bereich beteiligt und gehört werden. Deren Wahrnehmungen und Bedürfnisse zu berücksichtigen, bildet eine entscheidende Säule, wenn es darum geht, die Gesellschaft auf eine ältere und pflegebedürftigere Bevölkerung einzustellen. Schon länger kämpft die Allgemeinmedizin mit Nachwuchssorgen (Klose et al. 2003) und auch bei den derzeitigen Hausärzten findet sich keine flächendeckende Motivation zur Versorgung von Heimpatienten. Für diese ist eine gesicherte ärztliche Versorgung jedoch essentiell. Es gilt also, bestehende Rahmenbedingungen zu optimieren.

Aufgrund der dargestellten Aspekte soll mit der vorliegenden Arbeit untersucht werden, welche Wahrnehmungen und Bedürfnisse von Hausärzten hinsichtlich ihrer Tätigkeit im Pflegeheim bestehen. 


\section{Stand der Forschung}

Zunächst soll die allgemeine Situation in Deutschland beleuchtet werden. Hierbei werden Aspekte wie Pflegeheimversorgung und Versorgungsbedarf im Zeichen des demografischen Wandels, hausärztliche Versorgung und Vergütung in Zahlen und Fakten sowie einzelne Studien zu Modellprojekten in der heimärztlichen Versorgung vorgestellt. Des Weiteren liefert dieser Teil einen Überblick über bestehende Studien und Veröffentlichungen zur Arztperspektive in Deutschland. Hierbei werden das ärztliche Erleben der Arbeit, die Arbeitszufriedenheit und das Befinden der Ärzte unter den bestehenden Arbeitsbedingungen fokussiert betrachtet. Des Weiteren wird die Sicht der Ärzte auf die Arbeit im Heim dargestellt. Abschließend gibt dieser Promotionsteil einen Einblick in die internationale ärztliche Pflegeheimversorgung und das Erleben der Arbeit im Heim im internationalen Setting.

\subsection{Allgemeine Situation in Deutschland}

Demografischer Wandel, Multimorbidität und deren Einfluss auf den Versorgungsbedarf

Das Robert Koch-Institut legt in der Ausgabe zur Gesundheitsberichterstattung vom Februar 2012 dar, dass der demografische Wandel der deutschen Bevölkerung mit einer Zunahme an Multimorbidität (Vorliegen von mehreren chronischen Erkrankungen bei einer Person) einhergeht. Über die Hälfte der Personen über 65 Jahre weist mindestens eine chronische Erkrankung auf (Nowossadeck 2012). Chronische Erkrankungen sind wiederum eng mit Pflegebedürftigkeit verbunden (Blüher et al. 2017). Mit steigendem Lebensalter steigen das Krankheitsrisiko und somit der Versorgungsbedarf an. Damit nimmt auch der Gesamtversorgungsbedarf der deutschen Bevölkerung zu, da das Lebensalter der deutschen Bevölkerung kontinuierlich ansteigt.

Für 2030 wird davon ausgegangen, dass auf 100 Personen zwischen 20 und 65 Jahren, etwa 60 ältere Menschen (über 65 Jahre) kommen (Statistisches Bundesamt 2009). Gleichzeitig wird die Zahl derer, die diesen Versorgungsbedarf decken könnten, abnehmen (Nowossadeck 2012). Das Robert KochInstitut sieht die medizinische Forschung in der Pflicht, den aus der demografi- 
schen Entwicklung resultierenden Herausforderungen zu begegnen und zu einer verbesserten Versorgung beizutragen (Nowossadeck 2012).

Zu den Auswirkungen des demografischen Wandels auf das Gesundheitswesen äußert sich auch der Sachverständigenrat zur Begutachtung der Entwicklung im Gesundheitswesen. Er hält in seinem Sondergutachten 2009 zur Gesundheitsversorgung in einer Gesellschaft des längeren Lebens fest, „[...] dass die mit dem absehbaren demografischen Wandel einhergehenden Verschiebungen des Morbiditätsspektrums zielgerichtete Veränderungen der bisherigen Strukturen im deutschen Gesundheitswesen erfordern" (Deutscher Bundestag 2009, S. 27).

\section{$\underline{\text { Pflegeheime in Deutschland }}$}

Ende 2015 gab es in Deutschland rund 13.600 Pflegeheime, die der Definition des SGB XI zu Pflegeheimen entsprachen, davon 53\% in freigemeinnütziger Trägerschaft und $42 \%$ unter privaten Trägern. Öffentliche Heime machten nur $5 \%$ der Trägerschaften aus (Statistisches Bundesamt 2017). Im SGB XI werden Pflegeheime wie folgt definiert:

„(2) Stationäre Pflegeeinrichtungen (Pflegeheime) im Sinne dieses Buches sind selbständig wirtschaftende Einrichtungen, in denen Pflegebedürftige:

1. unter ständiger Verantwortung einer ausgebildeten Pflegefachkraft gepflegt werden,

2. ganztägig (vollstationär) oder tagsüber oder nachts (teilstationär) untergebracht und verpflegt werden können."

(Bundesministerium der Justiz und Verbraucherschutz 2017, SGB XI, § 71 Pflegeeinrichtungen, Abs. 2)

Pflegeeinrichtungen sind damit hauptsächlich für die Pflege der Bewohner zuständig. Der medizinische Versorgungsbedarf von Heimbewohnern ist jedoch auch sehr hoch und aus den bereits erläuterten Gründen der Multimorbidität und des demografischen Wandels weiter zunehmend. 


\section{Die ärztliche Versorgung von Pflegeheimbewohnern}

Die angesprochenen chronisch und mehrfach erkrankten sowie immer älteren Patienten werden ambulant hauptsächlich von hausärztlich tätigen Ärzten versorgt. Auf die „nachhaltige Funktionsfähigkeit“ der hausärztlichen Versorgung müsse deshalb besonders geachtet werden, „um die gesundheitlichen Belange der Bevölkerung in Deutschland sichern zu können“ (Deutscher Bundestag 2009, S. 31).

Die Patienten in einem Pflegeheim werden im Allgemeinen von einer Vielzahl verschiedener Ärzte versorgt. Es werden hierzu Zahlen von im Durchschnitt 23 Ärzten pro Heim genannt; davon 12 Allgemeinärzte und 11 Ärzte anderer Fachrichtungen (van den Bussche et al. 2009). Grundlage dessen ist die in Deutschland bestehende freie Arztwahl nach § 43 SGB XI, die auch im Pflegeheim gilt.

\section{Vergütungssystem}

Für Hausärzte ist die Vergütungssituation der hausärztlichen Tätigkeit ein wichtiges Thema. Die arztgruppenspezifischen Abrechnungsmaßstäbe für die Hausärzte sind in der folgenden Übersicht auszugsweise dargestellt. Hierbei wurden besonders die für die Versorgung von Heimbewohnern relevanten Punkte herausgestellt: 


\begin{tabular}{|l|l|}
\hline Versichertenpauschale $55-75$ Jahre (pro Quartal) & $16,73 €$ \\
\hline Versichertenpauschale $>76$ Jahre (pro Quartal) & $22,37 €$ \\
\hline Chronikerpauschale (pro Quartal) & $13,85 €$ \\
\hline Besuch & $22,59 €$ \\
\hline Mitbesuch & $11,29 €$ \\
\hline Dringender Besuch im Heim, am selben Tag & $58,17 €$ \\
\hline $\begin{array}{l}\text { Dringender Besuch bei Unterbrechen der Sprech- } \\
\text { stundentätigkeit mit Verlassen der Praxisräume }\end{array}$ & $66,70 €$ \\
\hline
\end{tabular}

Tabelle 1: Hausärztliche Vergütung

Zahlen aus (Kassenärztliche Bundesvereinigung 2018)

\begin{tabular}{|l|l|}
\hline Weg 8-20 Uhr, bis $2 \mathrm{~km}$ (Niedersachsen) & $3,57 €$ \\
\hline Weg 8-20 Uhr, 10 bis $15 \mathrm{~km}$ (Niedersachsen) & $13,71 €$ \\
\hline
\end{tabular}

Tabelle 2: Wegpauschalen Niedersachsen

Zahlen aus (Kassenärztliche Vereinigung Niedersachsen 2018)

\section{Wirtschaftlichkeitsprüfung}

Resultierend aus der aktuellen Vergütungssituation muss sich auch mit dem Thema der Wirtschaftlichkeitsprüfung für die hausärztliche Tätigkeit beschäftigt werden. Denn zusätzlich zu der von den Hausärzten wahrgenommenen unzureichenden Vergütungssituation sind Regressforderungen für niedergelassene Ärzte ein sowohl wirtschaftlich als auch emotional wichtiges Thema.

Die Regelungen zur Wirtschaftlichkeitsprüfung und den daraus ermittelten Regressansprüchen beziehen sich unter anderem auf das Wirtschaftlichkeitsgebot aus dem fünften Buch des Sozialgesetzbuches (Bundesministerium der Justiz 
und für Verbraucherschutz 2017, SGB V). Zu den Leistungen, die ein Arzt zu erbringen hat, wird hier erklärt: „Die Leistungen müssen ausreichend, zweckmäßig und wirtschaftlich sein; sie dürfen das Maß des Notwendigen nicht überschreiten. Leistungen, die nicht notwendig oder unwirtschaftlich sind, können Versicherte nicht beanspruchen, dürfen die Leistungserbringer nicht bewirken und die Krankenkassen nicht bewilligen" (Bundesministerium der Justiz und für Verbraucherschutz 2017, SGB V, § 12 Wirtschaftlichkeitsgebot, Abs. 1).

Bislang galt, dass Ärzte, die ein bestimmtes Richtgrößenvolumen bei der Verordnung von Arznei- und Heilmitteln überschritten, mit einer Wirtschaftlichkeitsprüfung zu rechnen hatten (Bundesministerium der Justiz und für Verbraucherschutz 2017, SGB V, § 84, Abs. 6, alte Fassung). Mit dem Versorgungsstärkungsgesetz von 2015 sollten auch hinsichtlich dieses Punktes Veränderungen vorgenommen werden (Deutscher Bundestag 2015). Eine Wirtschaftlichkeitsprüfung wurde jedoch zunächst auch weiterhin eingeleitet, wenn das RichtgröBenvolumen um mehr als 15\% überschritten wurde. Wurde das Richtgrößenvolumen um mehr als $25 \%$ überschritten, so konnte die eingesetzte Prüfungskommission zu der Entscheidung kommen, dass der Arzt den Krankenkassen die überschrittenen Mehrausgaben zurückzuzahlen hat. Ab dem 01.01.2017 wurden die Regelungen regional auf die Kassenärztlichen Vereinigungen und Spitzenverbände der Krankenkassen übertragen. Festzuhalten bleibt jedoch, dass sich die Kassenärztlichen Vereinigungen der Länder - dem jetzigen Verfahrens- und Kenntnisstand entsprechend - im Wesentlichen an den vormals geltenden bundesweiten gesetzlichen Regelungen zu orientieren scheinen, so z. B. auch die KV Niedersachsen (Kassenärztliche Vereinigung Niedersachsen 2017).

\section{$\underline{\text { Herausforderungen für Pflegekräfte und Hausärzte }}$}

In seinem Gutachten geht der Sachverständigenrat von einem Zuwachs der pflegebedürftigen Menschen in Deutschland um 94\% bis zum Jahr 2050 und damit von einer Gesamtzahl von 4,35 Millionen Pflegebedürttigen aus (Deutscher Bundestag 2009). Hinzu käme ein steigender Bedarf an professioneller Pflege. Das Robert Koch-Institut geht davon aus, dass dieser Bedarf allein durch das Personalangebot in den Pflegeberufen bereits ab dem Jahr 2018 
nicht mehr gedeckt werden kann (Nowossadeck 2012). Dem steigenden Bedarf an Gesundheitsleistungen steht eine schrumpfende und alternde Kohorte an Leistungserbringern gegenüber (Klose et al. 2003; Nowossadeck 2012), wodurch sich die Versorgungssituation verschlechtert. In der hausärztlichen Versorgung besteht das Problem des Nachwuchsmangels schon länger. Besonders für strukturschwache und ländliche Gebiete wird dieser zunehmend zum Problem (Klose et al. 2003). Im Bereich der stationären Versorgung werden nötige Verbesserungen vor allem in der Anzahl, Qualifikation und Professionalisierung von Pflegekräften gesehen. Aber auch die Notwendigkeit neuer pflegerischer Konzepte und Modelle wird festgestellt (Nowossadeck 2012).

\section{Vorstellung einzelner Studien zu Modellprojekten}

Studien und Ansätze zu Modellprojekten zur ärztlichen Versorgung in Pflegeheimen gab es in den letzten Jahren immer wieder. Eine der bedeutsamsten und größten Studien zur ärztlichen Versorgung von Pflegeheimbewohnern der letzten Jahre ist die SÄVIP-Studie von 2005 (Hallauer et al. 2005). Durch Fragebogenerhebung wurden Ergebnisse von 782 Heimen aus 16 Bundesländern gewonnen. Die Pflegeheime wurden aufgefordert, Angaben zur Struktur- und Prozessqualität der ärztlichen Versorgung im Heim zu machen. Die ärztliche Versorgung im Heim wurde zum größten Teil durch niedergelassene Ärzte, besonders durch Fachärzte für Allgemeinmedizin, geleistet. Angestellte Heimärzte gab es nur in 5\% der Heime. Mit einer Besuchshäufigkeit von $72 \%$ wöchentlichen oder mehr Besuchen hatten Allgemeinmediziner den größten Anteil an der Versorgungsfrequenz innerhalb der niedergelassenen Ärzte. Die Stellung des Hausarztes in der medizinischen Versorgung von Heimbewohnern wird damit noch einmal deutlich (Hallauer et al. 2005).

Einen ähnlichen Ansatz wie die SÄVIP-Studie verfolgte eine 2012 durchgeführte Studie der Johanniter-Unfall-Hilfe. Dabei wurden 90 Pflegeheime der Johanniter-Unfall-Hilfe zur ärztlichen Versorgungsituation in ihren Heimen befragt (Carrier und Warschau 2012). Die Ergebnisse aus 69 Heimen zeigten, dass die ärztliche Versorgung von Heimbewohnern im ländlichen Raum starke Unterschiede zur Versorgung in städtischen Gebieten aufweist. So wurde in städtischen und großstädtischen Einrichtungen in 60 bzw. 69\% der Heime eine wö- 
chentliche Visite durchgeführt, in den ländlich gelegenen Heimen kam es dagegen in keinem der befragten Häuser zu einer wöchentlichen Visite. Die Gewinnung von Hausärzten für die Arbeit im Heim gestaltete sich im ländlichen Raum schwieriger. Auch auf gesetzliche Reformen wurde in der Befragung eingegangen. Die nach dem Pflege-Neuausrichtungs-Gesetz angestrebten Kooperationsverträge zwischen niedergelassenen Ärzten und Pflegeheimen lagen lediglich in 4,3\% der Heime vor (Carrier und Warschau 2012). In den letzten Jahren wurden einige weitere Modellprojekte auf den Weg gebracht. In BadenWürttemberg wurde ein Vertrag zur Integrierten Versorgung mit drei Heimträgern geschlossen. Teilnehmende Hausärzte bildeten dabei ein Versorgungsnetzwerk untereinander und mit den teilnehmenden Pflegeheimen. Hausärzte verpflichteten sich unter anderem zur Teilnahme an Bereitschaftsdiensten werktags zwischen 7 und 19 Uhr und zu regelmäßigen Heimvisiten im mindestens 14-tägigen Rhythmus. Der ärztlich geleistete Mehraufwand sollte mit einer verbesserten Vergütung ausgeglichen werden (Sharp 2011).

Auch das Berliner Modellprojekt „Die Pflege mit dem Plus“ zielt auf eine verbesserte Kooperation zwischen Pflegeheim und Hausarzt ab. Die dort geschlossenen Kooperationsverträge zwischen niedergelassenen Ärzten und Heimen beinhalten eine 24-stündige Rufbereitschaft, wöchentliche Heimvisiten und regelmäßige interdisziplinäre Teambesprechungen (Hibbeler 2007). Für diese Leistungen erhalten die Ärzte ein zusätzliches Honorar von 200 Euro pro Patient und Quartal. Den entstehenden Mehrkosten durch dieses zusätzlich aufgebrachte Honorar steht eine Einsparung für die Krankenkassen von 2,7 Millionen Euro im Jahr 2005 gegenüber. Dies konnte durch die Reduzierung von Krankenhauseinweisungen und die sinkenden Arzneimittelausgaben, die durch die bessere hausärztliche Versorgung entstanden, erreicht werden. Ein weiteres Ziel dieses Modellprojektes: Ein Arzt soll möglichst viele Patienten in einem Heim betreuen. Durch die entstehende Kontinuität und Präsenz des Arztes im Heim soll auch die Zusammenarbeit zwischen Arzt und Pflegepersonal verbessert werden (Hibbeler 2007).

Neben der Herausforderung, den strukturellen Gegebenheiten und Veränderungen zu begegnen, besteht die Notwendigkeit einer Innenansicht auf das Er- 
leben und die Bedürfnisse der handelnden Personen. Wenn dabei die Person des Hausarztes in den Fokus gerückt wird, kann ein umfassendes Bild der Arbeitssituation von Hausärzten, speziell in der Versorgung von Pflegeheimbewohnern entstehen. Dieser Notwendigkeit wird mit der vorliegenden Arbeit und den untersuchten Fragestellungen begegnet.

\subsection{Arztperspektive in Deutschland}

\section{Gesamtzufriedenheit}

Die ärztliche Arbeits- und Berufszufriedenheit ist nicht nur von individueller, sondern auch von großer gesellschaftlicher Bedeutung, denn: „Ärzte sind eine Schlüsselgruppe, um ein gut funktionierendes Gesundheitssystem zu gewährleisten." (Arnetz 2001, S. 203). Die Arbeitsbedingungen von Ärzten beeinflussen sowohl die eigene Gesundheit der Ärzte als auch deren Motivation und damit die Qualität ihrer ärztlichen Versorgung (Angerer und Schwartz 2010). Aus belastenden Arbeitsbedingungen können eine abnehmende Versorgungsqualität und damit negative Ergebnisse auf Patientenseite resultieren (Gothe 2010). Dass mangelnde Berufszufriedenheit mit einer höheren Rate an niedriger Versorgungsqualität und Unzufriedenheit auf Patientenseite einhergeht, wird auch von anderen Autoren bestätigt (van den Hombergh et al. 2009; Löffler et al. 2014).

Den Einfluss von arztseitigen Faktoren auf die Berufszufriedenheit und wiederum deren Auswirkung auf die Patientenversorgung wurde von Gothe mit seiner Arbeit „Arbeits- und Berufszufriedenheit von Ärzten - eine Übersicht der internationalen Literatur“, mit dem Schwerpunkt „Physician Factor“, untersucht. Zu diesen arztseitigen Faktoren werden unter anderem Berufszufriedenheit, wahrgenommene Autarkie und Selbstwirksamkeit gezählt. Die eingeschlossenen 77 Publikationen ergaben, dass unter anderem hoher Leistungs- und Qualitätsdruck und arbeitsbezogene Belastungen zu einer geringen Arbeitszufriedenheit beitragen (Gothe 2010). Insgesamt zeigte sich in einem Review von elf Studien aus Deutschland bzw. von sich auf Deutschland beziehenden Studien aber auch, dass niedergelassene Ärzte eine hohe Gesamtarbeitszufriedenheit aufweisen. Diese wird durch erlebte Sinnhaftigkeit des ärztlichen Handelns, positi- 
ve Arzt-Patienten-Beziehungen, gutes Betriebsklima in der eigenen Praxis, Kollegialität und positives gesellschaftliches Ansehen gefördert (van den Bussche 2010). Auch andere Studien aus verschiedenen Teilen Deutschlands kommen zu guten Ergebnissen die Gesamtzufriedenheit betreffend (Schäfer et al. 2005; Behmann et al. 2012).

In einer in Mecklenburg-Vorpommern durchgeführten Fragebogenanalyse aus dem Jahr 2014 wird die Gesamtzufriedenheit sogar insgesamt als hoch bis sehr hoch angegeben (Löffler et al. 2014). Ziel dieser Studie war es unter anderem, die Ergebnisse aus einer vorherigen Studie über die Berufszufriedenheit von Allgemeinärzten in Mecklenburg-Vorpommern von 2005 auf ihre Aktualität hin zu überprüfen. Die Rücklaufquote sowie die Gesamtzahl an Teilnehmern war bei der Studie aus 2014 etwas geringer (50,1\% Rücklaufquote und 568 Teilnehmer gegenüber 64,6\% Rücklaufquote und 620 Teilnehmer) (Schäfer et al. 2008; Löffler et al. 2014). Den positiven Ergebnissen die Gesamtzufriedenheit betreffend aus dem Jahr 2014 steht eine als eher mäßig beschriebene Berufszufriedenheit der Hausärzte der vorhergehenden Studie gegenüber (Schäfer et al. 2008). Die Berufszufriedenheit scheint in der Gruppe der jüngeren Hausärzte (29 bis 45 Jahre) am höchsten und nimmt im Laufe des Berufslebens eher ab (Behmann et al. 2012; Löffler et al. 2014).

Im Vergleich zu ihren europäischen Kollegen sind deutsche Hausärzte insgesamt eher weniger zufrieden (Behmann et al. 2012). So weisen zum Beispiel französische und norwegische Allgemeinmediziner eine höhere Gesamtzufriedenheit auf (Schäfer et al. 2005; Voltmer et al. 2012). Interessant ist hierbei, dass die befragten französischen Hausärzte überwiegend in Gemeinschaftspraxen tätig waren, während die befragten deutschen Hausärzte überwiegend in Einzelpraxen arbeiteten (Schäfer et al. 2005). Auch in einer weiteren Studie kommen die Forscher zu dem Ergebnis, dass Ärzte in Gemeinschaftspraxen insgesamt zufriedener sind (Schäfer et al. 2008).

Zusammenfassend lässt sich sagen, dass die Gesamtzufriedenheit der deutschen Hausärzte anhand der bestehenden Studienlage als überwiegend gut eingeschätzt wird, im internationalen Vergleich jedoch eine schwächere Position einnimmt. 
Möchte man die Berufszufriedenheit von (Haus-) Ärzten genauer betrachten, so muss man die Zufriedenheit unter anderem hinsichtlich einzelner relevanter Aspekte untersuchen. Hierbei ergibt sich ein durchaus inhomogenes Bild:

\section{Finanzielle Rahmenbedingungen}

Die Vergütungssituation der Hausärzte wurde als Hauptproblem in einer Befragung von Hausärzten genannt (Kalitzkus 2011). Die finanzielle Situation der Hausärzte in Deutschland wird von den Betroffenen insgesamt als eher schlecht bezeichnet. Es bestehen hierbei Unterschiede innerhalb Deutschlands (Schäfer et al. 2005). Niedrige Zufriedenheit herrscht beispielsweise bei Hausärzten im Nordosten Deutschlands bezüglich der finanziellen Vergütung (Schäfer et al. 2008). Demgegenüber zeigte sich die Vergütungssituation der Hausärzte in einer weiteren Befragung in Niedersachsen mit eher durchschnittlichen Zufriedenheitswerten belegt. Ältere Hausärzte waren bezüglich der Vergütungssituation unzufriedener als ihre jüngeren Kollegen (Behmann et al. 2012). Ein Selektionsbias hinsichtlich des Antwort- und Teilnahmeverhaltens an Studien von eher unzufriedenen Hausärzten sollte in Betracht gezogen werden.

Die Vergütungssituation von Hausärzten wird sehr häufig in Interviews oder Befragungen thematisiert. Trotz des hohen Stellenwertes dieses Aspektes für die Hausärzte konnte gezeigt werden, dass der Einfluss auf die Gesamtberufszufriedenheit eher gering zu sein scheint (Gothe 2010; Behmann et al. 2012). Es wird angenommen, dass eine positive finanzielle Situation nur in Kombination mit anderen positiven Faktoren, wie hoher Arbeitsautonomie und guten kollegialen Verhältnissen, zu einer Zunahme der Arbeitszufriedenheit beitragen könne (Gothe 2010). Löffler et al. widersprechen dieser Ansicht. Laut den Ergebnissen der von ihnen durchgeführten Fragebogenanalyse habe die Vergütung einen deutlichen Einfluss auf die Berufszufriedenheit (Löffler et al. 2014). Zu differenzieren ist, ob es sich bei dem Meinungsbild zur Vergütung um die Einkommenshöhe und/oder die wahrgenommene Gerechtigkeit und Angemessenheit, besonders im Vergleich zu anderen Fachärzten, handelt. Dies wird nicht in allen Studien deutlich. Andere Autoren betonen, dass der überwiegende Anteil der in ihre Studie eingeschlossenen Hausärzte die Vergütung als fair und angemessen empfindet (Löffler et al. 2014). Demgegenüber sehen einige 
Weiterbildungsärzte für Allgemeinmedizin genau in diesem Bereich zu große Unterschiede und Ungerechtigkeit und empfinden damit einhergehend eine Abnahme der Wertschätzung der hausärztlichen Arbeit (Niehus et al. 2008). Bezüglich der Vergütung kommt hinzu, dass viele Hausärzte sich durch die Angst vor Regressforderungen zusätzlich belastet sehen (Buddeberg-Fischer et al. 2007; Löffler et al. 2014).

Auch die Vergütung betreffend scheinen Hausärzte im europäischen Ausland, beispielsweise in Frankreich, eine höhere Zufriedenheit aufzuweisen (Schäfer et al. 2005).

\section{Bürokratie}

Neben der Vergütung zeigt sich besonders das Feld von Bürokratie und administrativen Tätigkeiten als Quelle von Missstimmung unter den Hausärzten. Der Umfang und die Zunahme des bürokratischen Aufwands erzeugen bei den meisten Hausärzten eine hohe Unzufriedenheit (Schäfer et al. 2008; Kalitzkus 2011; Koch et al. 2011; Behmann et al. 2012). Dies schließt auch die Unzufriedenheit mit dem bestehenden Abrechnungssystem mit ein (Schäfer et al. 2005). Es wird berichtet, dass die zeitliche Belastung durch bürokratische Tätigkeiten die Zeit für den eigentlichen Patientenkontakt verringere und darüber hinaus nicht zu einer Verbesserung der Versorgung beitrage (Kalitzkus 2011). Verringerungen des bürokratischen Aufwands seien nötig, um den Beruf des Hausarztes auch für zukünftige Generationen attraktiver zu machen (Behmann et al. 2012). Auch hier liefert die Studie von Löffler et al. abweichende Ergebnisse. Hier heißt es, dass der Bereich der administrativen Aufgaben zwar mit einer hohen Unzufriedenheit behaftet sei, dies jedoch keinen großen Einfluss auf die Gesamtzufriedenheit habe (Löffler et al. 2014).

\section{Arzt-Patienten-Verhältnis}

Das Arzt-Patienten-Verhältnis ist, besonders für Allgemeinmediziner, eine wichtige Säule der Berufsausübung (Wilm und in der Schmitten 2007). Der Wunsch nach den in der Allgemeinmedizin oftmals entstehenden langjährigen und engen Patientenbindungen ist ein Grund, warum sich Uniabsolventen für den Facharzt für Allgemeinmedizin entscheiden (Niehus et al. 2008). 91\% der Be- 
fragten bei Behmann et al. zeigten sich zufrieden bis sehr zufrieden mit dem Patientenkontakt. Es fällt auf, dass die Zufriedenheit mit der Dauer der Tätigkeit als Hausarzt zunimmt (Behmann et al. 2012). Bei Löffler et al. wird erwähnt, dass die Gesamtzufriedenheit der ärztlichen Tätigkeit gegenüber mit der Art der Arzt-Patienten-Beziehung korreliert und somit höher liegt, je besser das Verhältnis wahrgenommen wird (Löffler et al. 2014). Bezüglich der Zufriedenheit mit dem Patientenkontakt kommen die schon erwähnten Studien allesamt zu guten bis sehr guten Beurteilungen (Schäfer et al. 2008; Löffler et al. 2014). Der enge und langjährige Patientenkontakt fördert die Freude an der Berufsausübung für den Hausarzt (Buddeberg-Fischer et al. 2007).

\section{Multimorbidität und Fallzahlen}

Die Zunahme an multimorbiden Patienten und ein Anstieg der Fallzahlen insgesamt sind eine zusätzliche Belastung und Arbeitslast für die Hausärzte (Erler et al. 2010; Kalitzkus 2011). Die Zunahme an Patienten insgesamt ist auch deshalb problematisch für den Hausarzt, da der höhere Versorgungsbedarf nicht entsprechend besser vergütet wird (Kalitzkus 2011). Hierzu ist zu bemerken, dass eine Verdichtung an (multimorbiden) Patienten auch für sich genommen belastend sein kann, unabhängig von der Vergütung.

\section{Belastung}

Die allgemeine Arbeitsbelastung, der Umfang an vorhandener Zeit für Familie und Freizeit und das empfundene Stresslevel bei der Arbeitsausführung können weitere wesentliche Stressoren des allgemeinärztlichen Handelns darstellen. Diesen Komplex betreffend weisen Hausärzte oft eine eher unterdurchschnittliche bis mangelnde Zufriedenheit auf (Schäfer et al. 2005; Schäfer et al. 2008; Goetz et al. 2011; Löffler et al. 2014). Im internationalen Vergleich zeigt sich, dass deutsche Ärzte die meisten Patientenkontakte bei gleichzeitig kürzester Kontaktzeit aufweisen (Koch et al. 2011).

\section{$\underline{\text { Ansehen }}$}

Die Empfindungen zu Ansehen und Wertschätzung des hausärztlichen Berufs durch die Gesellschaft bilden einen wichtigen Faktor für Hausärzte. Hinsichtlich 
der Anerkennung durch die Gesellschaft sehen Hausärzte ein schwindendes Ansehen ihrer Profession und damit auch eine mögliche Abschreckung für zukünftige Ärzte (Natanzon et al. 2010). Auch im Vergleich zu anderen Fachrichtungen hat die Allgemeinmedizin oft ein schlechteres Ansehen (Niehus et al. 2008). Berufsverbände und Medien würden dieses Image durch negative Berichterstattungen zusätzlich fördern (Niehus et al. 2008). Bei Schäfer et al. liegen die Werte für das empfundene Ansehen in der Bevölkerung für Deutschland hingegen über dem Durchschnitt (Schäfer et al. 2005). In der gesellschaftlichen Entwicklung hin zu einer starken Förderung und Ausprägung von Spezialisierungen kann der Allgemein-Mediziner mit seiner Profession nur schwer bestehen (Buddeberg-Fischer et al. 2007).

\section{$\underline{\text { Autonomie }}$}

Die empfundene Arbeitszufriedenheit von Ärzten korreliert laut Gothe stark mit deren empfundener professioneller Autonomie. Diese professionelle Autonomie beinhaltet ein hohes $\mathrm{Maß}$ an diagnostischer und therapeutischer Entscheidungsfreiheit (Gothe 2010). Die Möglichkeit, die eigenen Fähigkeiten auszuschöpfen, beeinflusst die Gesamtzufriedenheit der Hausärzte in hohem Maße (Goetz et al. 2011). Die Freiheit in der Berufsausübung kann durch die zunehmende Reglementierung durch Leitlinien, Disease-Management-Programme oder Zertifizierungen als eingeschränkt empfunden werden (Niehus et al. 2008). 


\subsection{Arztperspektive auf die Arbeit im Heim in Deutschland}

Über die Sicht und das Empfinden von Hausärzten der Arbeit im Heim gegenüber existieren nur wenige und vornehmlich kleinere Studien. Die Arbeit im Heim beeinflussende Faktoren weisen Ähnlichkeiten zu den unter 2.2 aufgeführten Ansichten der Hausärzte auf. Hinzu kommen als Faktoren die Zusammenarbeit mit den Pflegekräften im Heim und die Arbeit mit der besonderen Patientenklientel der Heimbewohner.

\section{Blick der Hausärzte auf die Zusammenarbeit mit den Pflegekräften}

Die Zusammenarbeit mit den Pflegekräften ist ein zentrales Thema, wenn es sowohl um positive als auch um problematische Erfahrungen geht.

Knapp die Hälfte der Hausärzte (45,5\%) einer quantitativen Fragebogenerhebung unter 83 Haus- und Fachärzten empfanden die Kooperation mit den Pflegekräften als positiv. Von den befragten Ärzten wurde dabei als positiv rückgemeldet, dass sie sich in der Mehrzahl durch die Pflegekräfte in ihrer Arbeit wertgeschätzt fühlen und zudem oftmals von den Pflegekräften lernen könnten (Meyer-Kühling et al. 2015). Kritische Auseinandersetzungen über Verordnungen und Verantwortlichkeiten können die Zusammenarbeit jedoch belasten (Kalitzkus 2005). Auch die Unsicherheit und oftmals daraus resultierende unangebrachte Arztanforderungen oder auch für den Patienten nicht zwangsläufig nötige Krankenhauseinweisungen werden kritisiert (Anliker et al. 2005; KrügerBrand 2014). Als ein häufiges Problem die Zusammenarbeit betreffend wird von vielen Hausärzten die Verfügbarkeit und Erreichbarkeit der Pflegekräfte benannt (Meyer-Kühling et al. 2015). Auch die Qualität der fachlichen Kommunikation bereite Schwierigkeiten (Anliker et al. 2005).

Bezüglich Verbesserungsvorschlägen hinsichtlich der Zusammenarbeit sehen Ärzte einen besonderen Bedarf in der Vereinheitlichung von Informationswegen, einem Ausbau von Fallbesprechungen und Fortbildungen und einer Verbesserung der personellen Ressourcen (Meyer-Kühling et al. 2015). Insgesamt sind Hausärzte an einer guten Zusammenarbeit interessiert (Anliker et al. 2005). 
Versorgungsqualität und Kompetenzen der Pflegekräfte aus hausärztlicher $\underline{\text { Sicht }}$

Die Arbeit der Pflegekräfte wird sowohl bei Meyer-Kühling als auch in einer Umfrage der KV Berlin als überwiegend gut beurteilt (Schlitt 2009; Meyer-Kühling et al. 2015). Assistenzärzte in Weiterbildung zum Allgemeinmediziner bemängeln dagegen, dass das Pflegepersonal oftmals ungenügend ausgebildet sei (Niehus et al. 2008). Auf ungenügend ausgebildete Pflegekräfte und einen Mangel an Verantwortungsübernahme wird auch in anderen Studien hingewiesen (Laag et al. 2014). In einem (schweizer) Artikel über die Erfahrungen von Hausärzten in Pflegeheimen, unter anderem in der Region Deutschschweiz, wird von Hausärzten bemängelt, dass beispielsweise Missstände in der Verabreichung von Medikamenten bestünden (Anliker et al. 2005). In einer qualitativen Lokalstudie aus Nordrhein-Westfahlen wurde nach Problemen mit ambulanten Pflegedienstangestellten gefragt. Auch hier wurden Probleme mit der Qualität der Versorgung benannt (Kalitzkus 2005). Möglicherweise können hier Parallelen zur Kooperation mit Mitarbeitern der Pflegeheime gezogen werden.

Erwarten würden befragte Hausärzte, dass sie von den Pflegekräften problemorientiert und differenziert informiert werden und dass sich die Pflegekräfte aktiv in die Versorgung mit einbringen (Anliker et al. 2005).

\section{Bürokratie}

Der bürokratische und administrative Aufwand wird auch bei einer KV-Umfrage unter Haus- und Fachärzten als problematisch angesehen. Die bürokratischen Anforderungen steigen durch das Bedürfnis von Seiten des Pflegeheims nach Absicherung an (Laag et al. 2014). Hinsichtlich des administrativen Aufwands wird eine große Notwendigkeit von Verbesserungen gesehen (Schlitt 2009). Erste Schritte hierzu sind in einzelnen Pflegeheimen bereits erfolgt. So wird von positiven Erfahrungen mit einem vernetzten elektronischen Dokumentationssystem berichtet (Krüger-Brand 2014). Hausärzte können hierbei von der Praxis aus auf die Dokumentation im Heim zugreifen, mit den Pflegekräften schriftlich kommunizieren und Anordnungen treffen. Die hierbei gewonnene Zeit könne wiederum in die Patientenversorgung investiert werden. Auch Krankenhaus- 
einweisungen konnten auf diesem Wege reduziert werden (Krüger-Brand 2014).

\section{Vergütung}

Den höchsten Verbesserungsbedarf sahen die von der KV Berlin befragten Ärzte in der finanziellen Vergütung (Schlitt 2009). Der zeitliche Mehraufwand durch die Versorgung von Heimbewohnern werde nicht entsprechend finanziell ausgeglichen (Kalitzkus 2011; Sharp 2011). Würde die Vergütungssituation verbessert, könnte dies laut den befragten Hausärzten auch die Bereitschaft zu Besuchen steigern (Kalitzkus 2011).

\section{Sicht auf die Heimbewohner}

Pflegeheimbewohner werden als besondere Kohorte wahrgenommen. Die Patientenklientel im Heim ist von Multimorbidität geprägt und sowohl Kommunikation als auch Mitarbeit beim Hausbesuch sind oft erschwert (Krüger-Brand 2014). Die Betreuung von Heimpatienten wird als aufwendig empfunden (Sharp 2011).

\section{Gesamteindruck}

In ihrer qualitativen Arbeit zur Einstellung von Hausärzten bezüglich Haubesuchen wurde von Theile et al. auch die Sicht von Hausärzten auf die Hausbesuche im Heim erhoben (Theile et al. 2011). Hierbei ergab sich ein überwiegend negativ geprägtes Bild. Die interviewten Hausärzte beschrieben, dass Pflegeheime von Verzweiflung, Einsamkeit und einer unangenehmen Atmosphäre geprägt seien (Theile et al. 2011).

\subsection{Internationale Perspektive}

Die hausärztliche Versorgung in Pflegeheimen ist auch im internationalen Rahmen von großer Bedeutung. Die Schwierigkeiten und Probleme, welche die Arbeitsbedingungen der Ärzte im Heim beeinflussen, sind dabei oftmals ähnlich denen in Deutschland, obwohl die Rahmenbedingungen international vielfach anders gestaltet sind und oftmals auch eine höhere professionelle Autonomie der Pflegekräfte vorliegt. 
Die ärztliche Perspektive auf die Arbeit im Heim und das Erleben sowie die Auswirkungen der Arbeit im Heim für den Hausarzt soll anhand ausgewählter Studien näher betrachtet werden.

\section{Blick der Hausärzte auf die Zusammenarbeit mit den Pflegekräften}

In einer schwedischen Studie sahen Hausärzte die Pflegekräfte als Schlüsselfaktor, um ihre Wünsche nach guter Versorgung und Wohlbefinden der Bewohner umzusetzen. Viele Hausärzte empfanden Abhängigkeit in ihrer Arbeitsumsetzung von den Pflegekräften (Bolmsjö et al. 2015). Auch in anderen Studien wird berichtet, dass examinierte Pflegekräfte eine wichtige Rolle bei der Möglichkeit der Umsetzung der ärztlichen Aufgaben spielen (Kirsebom et al. 2016). Viele Hausärzte berichten von einer Arbeitserleichterung und Bereicherung durch die Pflegekräfte (Bolmsjö et al. 2015). Hausärzte können wichtige Informationen über die Patienten durch die Pflegekräfte erlangen und in einigen Fällen konnten Krankenhauseinweisungen durch die Arbeit der Pflegekräfte verhindert werden. Besonders dadurch, dass Hausärzte nicht täglich im Heim vor Ort sind, müssen sie sich auf die Informationen der Pflegekräfte verlassen (können) (Kirsebom et al. 2016). Gut organisierte Pflegestrukturen fördern die Effizienz des ärztlichen Handels und erlauben es den Hausärzten, sich stärker auf ihre ärztliche Tätigkeit zu fokussieren (Bolmsjö et al. 2015). Auch bei der Auswahl und Einschätzung, welcher Bewohner bei einer Visite durch den Hausarzt gesehen werden sollte, sind die examinierten Pflegekräfte die Schlüsselfiguren. Damit tragen die Pflegekräfte zu einer Arbeitserleichterung und optimierung für den Hausarzt bei. Bei ungeplanten Notfallkonsultationen sind die Hausärzte wesentlich auf die korrekte Einschätzung der Pflegekräfte und Kommunikation mit diesen angewiesen (Kirsebom et al. 2016). Diese unvorhergesehenen Heimbesuche werden von den Hausärzten als problematisch empfunden, da sie zeitlich mit inrer Tätigkeit in der ambulanten Patientenversorgung kollidieren können (Kirsebom et al. 2016). Verlässlichkeit und Kompetenz der Pflegekräfte scheinen allgemein wesentliche Faktoren zu sein (Bolmsjö et al. 2015; Kirsebom et al. 2016).

Ist die Kontinuität in der Versorgung durch Pflegekräfte nicht gegeben oder sind zu wenig examinierte Pflegekräfte für ein Heim vorhanden, so sehen Hausärzte 
darin Probleme in der Umsetzung ihrer Versorgung für die Bewohner (Kirsebom et al. 2016). Der Mangel an Pflegekräften im Heim und an zeitlichen Ressourcen in der Versorgung durch Pflegekräfte wird durch die Ergebnisse einer qualitativen niederländischen Studie auch aus Sicht der Pflegekräfte als kritische Größe gesehen (Oosterveld-Vlug et al. 2013).

Der Umfang der Arbeitsbelastung kann durch die Pflegekräfte beeinflusst werden. So berichtet die Mehrheit der Befragten in einer englischen Studie über aus ihrer Sicht teils unnötige Visitenanforderungen (Williams et al. 1992). In Bezug auf die Optimierung von Entscheidungsprozessen und um "unnötige“ Krankenhauseinlieferungen zu vermeiden, wird das Bedürfnis nach spezialisierten Pflegekräften geäußert (McDermott et al. 2012). Möglicherweise überflüssige Krankenhauseinweisungen sind auch in der internationalen Literatur rund um das Thema Versorgung von Pflegeheimbewohnern als konfliktreich zu erkennen (Kirsebom et al. 2013).

Versorgungsqualität und Kompetenzen der Pflegekräfte aus hausärztlicher Sicht

In einer irischen Studie gaben über $50 \%$ der dort befragten Hausärzte, die eine Vielzahl von Pflegeheimpatienten versorgten, an, dass sie bei ihrer Arbeit auf unterdurchschnittliche Pflege gestoßen seien. In der Mehrzahl der Fälle sei dies auf unzureichende Beurteilungsfähigkeiten der Pflegekräfte zurückzuführen (Gleeson et al. 2014). Auch eine frühere irische Studie kommt zu ähnlichen Ergebnissen (Corroon-Sweeney et al. 2009). Die Versorgungsqualität im Pflegeheimen wird als oftmals "suboptimal“ beschrieben (Katz et al. 2009). Generell empfanden die Hausärzte bei Kirsebom die Pflegekräfte als kompetent und erfahren (Kirsebom et al. 2016).

Als frustrierend empfanden es Ärzte, wenn fehlende pflegerische Ressourcen durch medikamentöse Behandlungen kompensiert werden sollten. Als Beispiel werden die Verschreibungen von Sedativa angesprochen, die in vielen Fällen durch mehr Zuwendung reduziert werden könnten. Hierbei kam es auch zu Kritik an den Pflegekräften, die ein solches Vorgehen zu oft zur Problemlösung wünschen würden (Bolmsjö et al. 2015). Andere Studien weisen auf die Gefah- 
ren von Antipsychotika speziell bei älteren Patienten hin und betonen, dass diese zu oft nicht indikationsgerecht eingesetzt werden würden und dass Pflegeheimbewohner, im Vergleich zu Personen, welche zu Hause versorgt werden, deutlich häufiger solche Medikamente verschrieben bekommen (Lenander et al. 2017). Im Pflegeheim erhält knapp die Hälfte der Bewohner eine medikamentöse Therapie mit Psychopharmaka (Thürmann 2017). Kritisch anzumerken bleibt, dass diese Medikamente von einem Arzt verordnet werden müssen und die (angemessene) Indikationsstellung somit auch in den Aufgabenbereich des (Haus-) arztes fällt.

\section{Versorgungsumfang seitens der Hausärzte}

In Schweden versorgt ein Allgemeinmediziner üblicherweise ein komplettes Pflegeheim. Anders ist die Situation beispielsweise im Vereinigten Königreich und in Deutschland. Hier ist die Arztbindung stärker ausgeprägt, so dass Bewohner auch nach einem Heimeinzug üblicherweise von ihrem bisherigen Hausarzt betreut werden. Im Vergleich zeigt sich, dass eine wöchentliche Visite im Heim in Schweden kontinuierlicher stattfindet als beispielsweise in Ländern des Vereinigten Königreichs. Dort scheinen Hausbesuche auf Anfrage die Regel zu sein. Forscher sehen die Arbeitsbelastung des einzelnen Hausarztes für die geringere Visitenfrequenz in diesen Ländern als verantwortlich an (Jacobs 2003). In einer älteren englischen Studie konnte gezeigt werden, dass Hausärzte, die ein komplettes Pflegeheim betreuen, eher Routinevisiten abhalten als Hausärzte, die nur einige Bewohner eines Heimes versorgen (Williams et al. 1992). Allgemeinmediziner, die während ihrer Ausbildungszeit bereits Pflegeheimbesuche durchgeführt haben, scheinen darüber hinaus eine höhere Bereitschaft zu zeigen, Pflegeheime auch im weiteren Verlauf ihrer Praxistätigkeit zu betreuen (Magin et al. 2017).

\section{Medizinische Komplexität}

Eine schwedische Studie stellte fest, dass insbesondere wenig erfahrene Hausärzte die Arbeit im Heim als besonders anspruchsvoll empfinden. Mit steigender Erfahrung nimmt auch die Sicherheit in der Versorgung zu (Kirsebom et al. 2016). Nichtsdestoweniger werden auch im internationalen Vergleich Her- 
ausforderungen durch die Arbeit mit der besonderen Patientenklientel der Heimbewohner gesehen (McDermott et al. 2012), was unter anderem auf die hohe Gebrechlichkeit und medizinische Komplexität zurückgeführt wird (Katz et al. 2009). Die kürzeren Versorgungszeiten in Krankenhäusern, beispielsweise in den USA, erhöhen die medizinische Komplexität der Pflegeheimbewohner zusätzlich. Bei Katz et al. wird dies mit dem Ausdruck sicker-but-quicker umfasst, was bedeutet, dass Patienten früher, aber auch kränker zurück ins Pflegeheim entlassen werden (DeFrances und Hall 2007; Katz et al. 2009). Die Versorgung von einer Vielzahl an Heimpatienten scheint die Einstellung der Arbeit im Heim gegenüber negativ beeinflussen zu können. So wird die Arbeit im Heim von den Hausärzten, die eine Vielzahl von Heimpatienten versorgen, in einer irischen Studie als „mühsam“ bzw. „beschwerlich“ empfunden (Corroon-Sweeney et al. 2009).

\section{Bedürfnisse und Wünsche der Hausärzte}

Die qualitative Studie von Bolmsjö et al. untersuchte die Erfahrungen von Allgemeinmedizinern mit der Versorgung von Pflegeheimbewohnern in Schweden. In dieser Studie, die auf semi-strukturierten Interviews mit Allgemeinmedizinern basiert, wurden das emotionale Erleben und die Sicht der Hausärzte in den Fokus gerückt. Das Wohlergehen der Patienten im Heim ergab sich als Hauptanliegen der Hausärzte. Hilflos fühlten sich Hausärzte, wenn sie den Anspruch nach ausreichender Versorgung der Bewohner nicht erfüllen konnten. Hieraus resultierte bei den Ärzten oftmals der Wunsch nach mehr Pflegekräften beziehungsweise mehr pflegerischer Versorgung (Bolmsjö et al. 2015). Als zwei Hauptthemen für die Hausärzte in der Interviewanalyse erwiesen sich die Sorge um die Patienten und die Beschäftigung mit tragfähigen Arbeitsbedingungen im Heim. So war es den Ärzten ein großes Anliegen, die besondere Patientengruppe der älteren Menschen in ihrer letzten Lebensphase gut versorgt zu wissen. Das ärztliche Anliegen galt der Reduzierung von Schmerz, Leid und Einsamkeit ihrer Patienten. Ihr Ziel war es, das bestmöglichste Wohlbefinden für ihre Patienten zu erreichen (Bolmsjö et al. 2015). Wünsche der Hausärzte sind darüber hinaus Verbesserungen in den Bereichen Kommunikation, Training des Pflegepersonals sowie Unterstützung und Ausbildung durch 
andere Hausärzte zu schaffen (McDermott et al. 2012). Hausärzte erwähnen, dass die Arbeitsbedingungen im Heim durch das Vorhalten von mehr medizinischen Materialien und Interventionsmöglichkeiten verbessert werden könnten. Als Beispiele werden unter anderem die wünschenswerte Möglichkeit von intravenösen Behandlungen und Laborabnahmen genannt. Auch wäre es wünschenswert, dass die Patientendokumentation und der Zugang zu dieser verbessert wird (Kirsebom et al. 2016).

Hansson et al., die sich mit dem allgemeinen Selbstverständnis von Hausärzten und ihrer professionellen Rolle befassten, konnten mehrere Themen aufdecken, die Hausärzten in ihrer täglichen Arbeit wichtig sind und über die sie sich selbst definieren. Für Hausärzte ist es demnach wichtig, die Balance zwischen der menschlichen, fürsorglichen Rolle und der professionellen medizinischen Rolle zu finden. Ein zusätzliches Thema ist, dass Gleichgewicht zwischen Heilung und Behandlung nicht kurativ behandelbar Erkrankter zu finden. Ein möglicher Konflikt kann für die Hausärzte dadurch entstehen, dass sie sich zwar in erster Linie den Patienten und deren Wohlergehen verpflichtet fühlen, jedoch auch eine gesellschaftliche Verantwortung tragen, welche Beschränkungen für die individuelle Patientenversorgung mit sich bringen kann (Hansson et al. 2007).

\section{Arbeitsbelastung durch Heimpatienten}

Bei Gleeson et al. wird bemerkt, dass die Besuche bei Heimpatienten im Vergleich zu Hausbesuchen komplexer und zeitaufwendiger seien und zudem der bürokratische Aufwand zunehmen würde (Gleeson et al. 2014). Bestätigt wird dies indirekt bei Groom et al. Hier kam man zu dem Ergebnis, dass die Arbeitsbelastung durch Heimpatienten im Vergleich zu einer ähnlichen Kohorte an zu Hause lebenden Patienten größer sei (Groom et al. 2000). Die höhere Arbeitsbelastung wurde an Kriterien wie der Anzahl der Hausbesuche und Telefonkontakte gemessen. Kritisch anzumerken ist, dass unklar bleibt, wie viele Patienten bei einem Heimbesuch gesehen wurden und inwieweit dies die höhere Arbeitsbelastung wieder ausgleichen könnte. Eine anonyme Umfrage unter Hausärzten in Dublin kommt ebenfalls zu dem Schluss, dass Heimbesuche zeitaufwendiger seien als Hausbesuche in der übrigen Bevölkerung (Corroon- 
Sweeney et al. 2009). In einer frühen englischen Studie wurde der Mehraufwand durch Heimpatienten folgendermaßen zusammengefasst: „t would appear that each nursing home patient was equivalent to three practice patients aged under 65 years" (Andrew 1988, S.547). Pell und Williams untersuchten die Inanspruchnahme von Hausärzten durch Heimpatienten und durch eine Vergleichsgruppe an zu Hause lebenden Patienten. Bei den Pflegeheimbewohnern hatten in dem beobachteten Zeitraum 77\% der Personen Kontakt zum Hausarzt. Demgegenüber ergab sich bei der Kohorte der nicht im Heim lebenden Patienten nur eine Inanspruchnahme des Hausarztes von 48\% (Pell und Williams 1999). In 90\% der Fälle erfolgte die Initiierung des Hausbesuches bei den Pflegeheimbewohnern durch eine Pflegekraft (Pell und Williams 1999). Es bleibt zu diskutieren, ob die höhere Arbeitsbelastung durch Pflegeheimpatienten rein durch deren erhöhten Bedarf an medizinischer Versorgung, oder auch durch eine höhere Inanspruchnahme und ein Absicherungsbedürfnis seitens der Pflegekräfte zu Stande kommt. In einer älteren englischen Studie wurde von vielen der dort befragten Hausärzte ebenfalls berichtet, dass die Arbeit im Heim die Arbeitsbelastung erhöhen würde. Der Großteil der Hausärzte empfand dieses Mehr an Arbeitsbelastung jedoch als handhabbar (Williams et al. 1992).

\section{Finanzielle Rahmenbedingungen}

Das Erleben der finanziellen Rahmenbedingungen wird in einem irischen Artikel angesprochen. Die Vergütung von Heimbesuchen hätte sich demnach über die Jahre verschlechtert. Hieraus könnte laut der Mehrzahl der befragten Hausärzte ein abnehmender Versorgungsumfang für die Heimpatienten resultieren (Gleeson et al. 2014). Groom et al. halten fest, dass die höhere Arbeitsbelastung durch Heimpatienten auch bei der Vergütung der Hausärzte einen Einfluss haben sollte (Groom et al. 2000).

\section{Erfahrungen und Eindruck die Arbeit im Heim betreffend}

Befragte Hausärzte empfanden ihre Arbeit als angenehm und bedeutsam. Sie empfanden, dass ihre Arbeit viel für die Bewohner im Heim erreichen kann. Darüber hinaus wurde die Arbeit im Heim und im Team mit den Pflegekräften 
als willkommene Abwechslung zur täglichen autonomen Arbeit in den praxisähnlichen Primary Health Care Centers gesehen. Hierbei schien für die Hausärzte die Freiheit im eigenen Zeitmanagement wichtig zu sein (Bolmsjö et al. 2015). Die Rahmenbedingungen im Heim bieten positive Chancen für die Hausärzte. So wird beispielsweise die Möglichkeit, einen allumfassenden Blick auf die Patienten zu erlagen, an der Arbeit im Heim geschätzt (Bolmsjö et al. 2015). Das Erlangen dieses „Gesamtbildes“ der Patienten macht die Arbeit für die Hausärzte befriedigender.

\section{Ausbildung und Zukunftsperspektiven}

Für die Zukunft äußerten schwedische Hausärzte den Wunsch nach mehr und regelmäßigem Austausch mit Kollegen und nach einer gründlicheren Einführung zu Beginn der Arbeit im Heim durch erfahrene Kollegen. Dieser Austausch sei derzeit noch nicht ausreichend vorhanden (Bolmsjö et al. 2015). Auch eine japanische Studie fordert den Ausbau der geriatrischen Ausbildung für hausärztliche Kollegen (Arai et al. 2012). Andere Autoren kommen zu dem Ergebnis, dass sich weniger als $15 \%$ der Assistenzärzte sehr gut auf die Aufgaben im Pflegeheim vorbereitet fühlen (Blumenthal et al. 2001). Dagegen fühlten sich $64 \%$ der Befragten einer irischen Fragebogenstudie ausreichend auf die Tätigkeit im Pflegeheim vorbereitet (Gleeson et al. 2014). Besonders sicher bezüglich ihrer Tätigkeit fühlten sich demnach die Ärzte, die eine Vielzahl an Heimpatienten versorgten (Gleeson et al. 2014). Corroon-Sweeney et al. kamen in ihrer früheren Umfrage zu ähnlichen Ergebnissen (Corroon-Sweeney et al. 2009).

Ein möglicher Lösungsvorschlag für die Verbesserung der heimärztlichen Versorgung kann in der Schaffung einer speziellen Fachrichtung bzw. Ausrichtung auf Pflegeheimpatienten gesehen werden. Eine zeitlicher Aufwand von mindestens $20 \%$ der Gesamttätigkeit bzw. vier Stunden pro Woche sollte hierbei grundsätzlich gegeben sein (Katz et al. 2009). Es bleibt fraglich, wie sich hierzu die Bereitschaft der Hausärzte darstellen würde.

\section{Gesamteindruck}

Das schwedische Forscherteam um Bolmsjö erwähnt in seiner Schlussfolgerung, dass der Wunsch nach mehr (pflegerischen) Ressourcen auch als Aus- 
druck angesehen werden kann, dass Hausärzte stetig gerne mehr für ihre Patienten tun würden, als es ihre Möglichkeiten zulassen.

Insgesamt konnte eine positive Grundstimmung der schwedischen Hausärzte der Arbeit im Heim gegenüber festgestellt werden (Bolmsjö et al. 2015). Die in der beschriebenen Studie interviewten Ärzte waren allesamt sehr interessiert an der Versorgung von Pflegeheimbewohnern und schätzten ihre Arbeit als interessant und wichtig ein (Bolmsjö et al. 2015). Auch Kirsebom et al. kommen zu einem positiven Gesamteindruck der Hausärzte gegenüber den Pflegeheimen. Die befragten Hausärzte waren sich darin einig, dass „nursing homes are places where older persons can end their life with dignity" (Kirsebom et al. 2016, S.4). 


\section{Fragestellung und Ziel}

Nach den Darstellungen zum Stand der Forschung wird deutlich, dass die Studienlage zur hausärztlichen Arbeit im Heim in Deutschland nicht ausreichend ist. Im Vergleich der Anteile zum Stand der Forschung aus deutscher und internationaler Sicht wird ersichtlich, dass insbesondere die Wahrnehmungen und Bedürfnisse von Hausärzten in der deutschen Forschungslandschaft nur wenig beleuchtet werden. Auch hinsichtlich hausärztlicher Erfahrungen mit der Arbeit im Heim besteht Forschungsbedarf. Hausärzte stellen eine wesentliche Säule in der Versorgung von Pflegeheimbewohnern dar. Eine umfangreiche Betrachtung ihrer Sichtweise zur Tätigkeit im Pflegeheim hat deshalb eine wichtige Bedeutung. Besonders qualitative Studien, die eine tiefergehende Analyse zulassen und detailreicher die subjektiven Wahrnehmungen hervorbringen können als quantitative Studien, sind im deutschen Stand der Forschung bezüglich der Arbeit im Heim rar. Die Notwendigkeit, dem Forschungsbedarf hinsichtlich der bestehenden Lücke gerecht zu werden, ist gegeben. Die vorliegende Promotionsarbeit soll dazu beitragen, diese Lücke zu schließen. Ziel der vorliegenden Arbeit ist es, durch die Bearbeitung der im Folgenden aufgeführten Fragestellungen wesentliche Aspekte der hausärztlichen Sicht hinsichtlich ihrer Tätigkeit im Pflegeheim aufzudecken und ein besseres Verständnis der ärztlichen Perspektive zu erlangen. Hierauf aufbauend können verbesserte Bedingungen für die im Pflegeheim tätigen Hausärzte geschaffen werden und somit Anreize bei Hausärzten für die Versorgung von Pflegeheimbewohnern gesetzt werden. Dies kann zu einer besseren Versorgung der Bewohner beitragen und auch die interprofessionelle Zusammenarbeit zwischen Ärzten und Pflegekräften optimieren. Eine bessere Kenntnis der Arztperspektive bildet somit eine entscheidende Grundlage, wenn es darum geht, die stationäre Pflegelandschaft Deutschlands zu verbessern.

Die mit den Hausärzten geführten Leitfadeninterviews sollen hinsichtlich folgender Fragestellungen untersucht werden: 
1. Welche Wahrnehmungen und Bedürfnisse werden von den interviewten Hausärzten bezüglich ihrer eigenen Arbeitssituation genannt? Welche Wünsche hinsichtlich einer Lösungsfindung werden geäußert?

Hierbei soll im Speziellen diese Fragestellung auf die Bereiche der bestehenden Heimstrukturen und Arbeitsbedingungen bei der Versorgung von Pflegeheimbewohnern angewandt werden.

2. Welche Wahrnehmungen und Bedürfnisse werden von den interviewten Hausärzten hinsichtlich der Versorgung und Betreuung der Bewohner genannt?

3. Welche Wahrnehmungen und Bedürfnisse bezüglich des Tätigkeitsbereichs Pflegeheim existieren? Wie wird das Arbeitsumfeld Pflegeheim wahrgenommen?

Die dritte Frage wurde entwickelt, um eine vollständige Darlegung der Wahrnehmungen und Bedürfnisse von Hausärzten zu erlangen. Mit dieser Fragestellung soll auch das emotionale Erleben der Ärzte berücksichtigt werden, da die von den Hausärzten genannten Wahrnehmungen und Wünsche schlussendlich das Ergebnis der professionellen Sicht der Ärzte auf ihre Arbeitssituation und deren emotionalem Erleben der Tätigkeit sind. 


\section{Material und Methoden}

\subsection{Methodische Anlage der Untersuchung und deren Einbettung in ein Drittmittelprojekt}

Die Promotionsarbeit erfolgt im Rahmen der Studie „INTERPROF - Interprofessionelle Zusammenarbeit und Kommunikation im Pflegeheim“. Die Studie wurde von folgenden Instituten gemeinsam durchgeführt:

- Institut für Allgemeinmedizin, Universitätsmedizin Göttingen (Studienleitung und Koordination)

- Institut für Allgemeinmedizin, Universitätsklinikum Eppendorf, Hamburg

- Arbeitsgruppe Psychiatrische Epidemiologie und Demografischer Wandel, Zentralinstitut für Seelische Gesundheit, Medizinische Fakultät Mannheim/Universität Heidelberg, Mannheim

- Abteilung Pflege und Gesundheit, Fakultät V, Hochschule Hannover

Die Studie war angesetzt für einen Zeitraum von drei Jahren. Beginn war im Februar 2012, es erfolgte eine halbjährige kostenneutrale Verlängerung bis Juli 2015. Die Studie wurde vom Bundesministerium für Bildung und Forschung gefördert (Förderkennzeichen FK 01GY 1124).

In der vierstufigen, qualitativen Multicenterstudie sollten Defizite und Potenziale der interprofessionellen Zusammenarbeit und Kommunikation zwischen Hausärzten und Pflegekräften sowie Bedürfnisse und Erwartungen von Hausärzten, Pflegekräften, Bewohnern und Angehörigen von Bewohnern im Pflegeheim aufgedeckt und analysiert werden. Hierauf aufbauend erfolgt die Entwicklung eines Kommunikations-/Kooperationsmodells, welches zur Verbesserung der medizinischen Versorgungsqualität in Pflegeheimen beitragen soll (Müller et al. 2015).

Im ersten Studienabschnitt wurden Leitfadeninterviews mit jeweils zehn Hausärzten, Pflegekräften, Bewohnern und Bezugspersonen pro Standort durchgeführt. Ziel war die Ergründung individueller Erfahrungen, Probleme, Bedürfnisse und Ansichten der einzelnen Beteiligten mit dem Fokus auf die medizinische 
Versorgung der Bewohner und die interprofessionelle Zusammenarbeit. Die Auswertung der Interviews erfolgte mittels der Methode der Grounded Theory (Strauss und Corbin 2010). Parallel zu den Interviews erfolgten fünf teilnehmende Beobachtungen von fünf ärztlichen Heimbesuchen in Pflegeheimen Göttingens. Im nächsten Schritt wurden insgesamt neun Fokusgruppen mit Hausärzten und Pflegekräften sowohl mono- als auch interprofessionell durchgeführt. Hierbei stand u. a. die Erarbeitung möglicher Lösungsstrategien zur besseren Zusammenarbeit im Fokus. Im Folgenden wurden diese Strategien in Expertenworkshops zu Kommunikations- und Zusammenarbeitsmodellen weiter differenziert und Strategien der Implementierung festgelegt. Abschließend wurde ein Maßnahmenpaket zur Verbesserung der interprofessionellen Kooperation und Kommunikation festgelegt. Abgeschlossen wurde die Studie im zweiten Teil mit der beispielhaften Implementierung des Modells in vier Pflegeheimen und dessen anschließender Evaluation mittels Interviews und kurzen quantitativen Fragebögen zu Akzeptanz und Machbarkeit.

Für das vorliegende Promotionsvorhaben wurden 30 Leitfadeninterviews mit in der hausärztlichen Versorgung von Pflegeheimbewohnern tätigen Ärzten geführt und nach der Methode der Grounded Theory ausgewertet. Auf Basis derselben Rohdaten wie die vorliegende Dissertation, entstand eine Arbeit von Fleischmann et al. (Fleischmann et al. 2016). Die qualitative Auswertung der Hausarztinterviews konzentrierte sich bei der Arbeit von Fleischmann et al. auf die Zusammenarbeit von Pflegekräften und Hausärzten beim Heimbesuch. Im Gegensatz hierzu standen für das vorliegende Promotionsverfahren die Wahrnehmungen und Bedürfnisse von Hausärzten hinsichtlich ihrer Tätigkeit im Pflegeheim im Fokus. Die vorliegende Dissertation entstand ohne Kenntnis des inhaltlichen Voranschreitens oder der Ergebnisse der Arbeit von Fleischmann et al. Auch erfolgte die Datenauswertung der Doktorandin für die vorliegende Arbeit unabhängig davon.

Für das Studiendesign der INTERPROF-Studie und die unter 3. dargestellten Forschungsfragen der vorliegenden Arbeit eignet sich das Vorgehen mit einer qualitativen Forschungsmethode wie der Grounded Theory besonders gut. Marx und Wollny erklären hierzu: „Vor allem für Forschungsfragen, die sich 
z. B. auf die Perspektive von ÄrztInnen oder PatientInnen beziehen, die die Arzt-Patient-Beziehung untersuchen oder die auf zugrunde liegende Konzepte wie Lebensqualität, Compliance oder Zufriedenheit gründen, sind qualitative Methoden besonders geeignet" (Marx und Wollny 2009a, S. 106). Im Gegensatz zur klassischen quantitativen Hypothesenbildung erlaubt der qualitative Ansatz, mit einer größeren Offenheit an das Material heranzutreten und aus den neuen Inhalten stärker aufgeschlüsselte Ergebnisse zu generieren. Während des gesamten Forschungsvorhabens bleibt der Forscher stärker mit dem Material verbunden und reflektiert sich und die gewonnenen Ergebnisse in einem stetigen Prozess. Die qualitative Analyse ermöglichte es bei der vorliegenden Arbeit, die tiefer liegende subjektive Wahrnehmung und den Handlungssinn der Interviewpartner herauszuarbeiten und zu analysieren. Auch galt es in dieser Arbeit, „methodisch kontrolliert herauszuarbeiten, aufgrund welcher Sinnbezüge gerade so gehandelt wurde, wie gehandelt wurde" (Schröer 1997, S.112).

Aufgrund der dargestellten Ziele und Ansprüche wurde für das Forschungsvorhaben der Gesamtstudie das offene Leitfadeninterview zur Datenerhebung und für die Datenanalyse und -interpretation der qualitative Forschungsansatz der Grounded Theory ausgewählt. Beide Vorgehensweisen erlaubten es, dass in der qualitativen Forschung etablierte Prinzip der Offenheit umzusetzen (Marx und Wollny 2009a; Flick 2010). Die Leitfadeninterviews und die Grounded Theory werden im späteren Teil dieser Dissertation näher beschrieben.

Im Folgenden wird nun die Vorgehensweise für die Gruppe der interviewten Hausärzte beschrieben.

\subsection{Rekrutierung der teilnehmenden Hausärzte und Einwilligungsge- spräch}

An den drei verschiedenen Standorten Göttingen, Mannheim und Hamburg wurden zunächst, angelehnt an das Prinzip des Purposive Sampling und später angelehnt an das Prinzip des Theoretical Samplings (Strauss und Corbin 2010), jeweils zehn in Pflegeheimen tätige Hausärzte rekrutiert. Purposive Sampling bedeutet, dass im Vorfeld bestimmte Kriterien aufgestellt wurden, die von den Interviewpartnern erfüllt werden sollten und nach denen diese ausge- 
sucht wurden. In unserem Falle waren dies Geschlecht, Anzahl von Patienten im Pflegeheim, Stadt- oder Landarzt. Die Rekrutierung erfolgte über Kontakte zu niedergelassenen Hausärzten der jeweiligen Institute an den drei Standorten, durch Nachfragen in teilnehmenden Pflegeheimen oder per Anschreiben in einem definierten Postleitzahlengebiet. Es wurden hausärztliche Praxen aus dem städtischen und ländlichen Einzugsgebiet der einzelnen Standorte ausgewählt und die ausgewählten Hausärzte angeschrieben. Die teilnehmenden Ärzte mussten darüber hinaus folgende Einschlusskriterien erfüllen:

- Tätigkeit als Hausarzt mit Versorgung von Bewohnern im Pflegeheim

- Schriftliche Einwilligung in die Studienteilnahme

- Ausreichende Deutschkenntnisse

Um die Vielschichtigkeit der Hausärzte abzubilden, war es gewünscht, Hausärzte mit wenigen, vielen oder überwiegend Patienten im Pflegeheim zu interviewen. Der Versuch, Männer und Frauen mit unterschiedlicher Tätigkeitsdauer im Pflegeheim zu rekrutieren, diente ebenfalls dem Zweck, Vielseitigkeit in den Ergebnissen abzubilden. Das Anschreiben (Anhang a) informierte die Ärzte kurz über die Studie und enthielt die Anfrage zu einem 60-minütigen Interview. Die Ärzte wurden darüber informiert, dass ihre Teilnahme eine Aufwandsentschädigung von 50 Euro beinhaltet. Bei erfolgter Zustimmung erhielten die Ärzte postalisch ein weiteres Informationsschreiben (Anhang b). Dieses beinhaltete Informationen zum Interviewablauf und Erklärungen zum Datenschutz. Zusätzlich erhielten die Ärzte vorab die Einverständniserklärung (Anhang c) zu Art und Speicherung ihrer Daten. Ort und Zeitpunkt des Interviewtermins wurde von den Ärzten gewählt. Die Unterschrift der Einverständniserklärung erfolgte am Interviewtag nach erneuter mündlicher Aufklärung durch den Interviewer.

Theoretical Sampling war in dieser Studie nur in Teilen umsetzbar, da die umfangreiche Interviewauswertung nicht immer vor dem Führen neuer Interviews abgeschlossen war. Theoretical Sampling bedeutet, dass die Interviewpartner nacheinander ausgewählt und kontaktiert werden und zwar auf der Basis der jeweils bei den vorherigen Interviews erhobenen Ergebnisse. Anhand der Analyse der vorangegangenen Interviews wird entschieden, was bei der weiteren 
Datenerhebung zu beachten ist. Das Sampling findet so statt, dass die nächsten Interviewpartner einen möglichst hohen Gehalt an neuen Aspekten liefern können. Die Datenerhebung wird abgeschlossen, wenn die „theoretische Sättigung" erreicht ist, also wenn keine neuen Aspekte mehr zu erwarten sind (Flick 2010; Strauss und Corbin 2010).

\subsection{Interviewleitfaden}

Für das Forschungsvorhaben wurde die Methode des offenen Leitfadeninterviews gewählt. Dabei wird anhand eines schriftlich festgehaltenen Leitfadens der Interviewpartner mit möglichst offen gestellten Fragen dazu angehalten, seine Perspektive darstellen und erzählen zu können. Dieses Vorgehen ermöglichte es, die für den Interviewten relevanten Themen konkreter zu erfassen und ein Bild seiner Ansichten und des Kontexts, in dem diese sich bewegen, zu erlangen (Przyborski und Wohlrab-Sahr 2010). Im April 2012 wurde mit der Konzipierung der Interviewleitfäden für die vier Expertengruppen begonnen. Die Generierung der Leitfäden erfolgte als mehrstufiger gruppendynamischer Prozess von an der Studie beteiligten Mitarbeitern unter Leitung von Dr. Christiane Müller, Institut für Allgemeinmedizin der Universitätsmedizin Göttingen und Dr. Gabriella Marx, Soziologin in der Abteilung für Palliativmedizin, Universitätsmedizin Göttingen. Der erarbeitete Interviewleitfaden für die Gruppe der Hausärzte (Anhang d) umfasste folgende Themenschwerpunkte:

1. Erzählung eines typischen Hausbesuchs im Pflegeheim

2. Beschreibung des letzten Hausarztbesuchs

3. Erfahrungen mit Zuständigkeitsbereichen und Aufgabenverteilungen

4. Vorstellungen und Phantasien über die ideale Situation/Versorgung im Pflegeheim

Die interviewten Ärzte wurden dazu angeregt, möglichst frei und offen zu den jeweiligen Punkten zu erzählen. Dennoch beinhalteten die Leitfragen auch konkretere Nachfragen, die weitere Denkanstöße beim Interviewpartner setzen sollten. Zu Beginn eines jeden Themenblockes stand jedoch immer eine erzählgenerierende Einstiegsfrage. Im Verlauf der Interviewerhebung wurden insbesondere die Nachfragen im Interviewleitfaden an die Beobachtungen und 
Ergebnisse der bereits erfolgten Interviews mehrfach angepasst. Die qualitative Forschung sieht dies als wichtiges Element vor, um der Variabilität der Interviewinhalte gerecht zu werden und entsprechende Ergebnisse zu generieren (Kalitzkus 2005; Wilm 2005; Strauss und Corbin 2010). Zum Abschluss eines jeden Interviews wurde dem Arzt die Möglichkeit zu abschließenden Ergänzungen gegeben.

\subsection{Durchführung der Untersuchung}

Ort und Zeitpunkt der Interviewdurchführung wurden nach Wunsch der Ärzte festgelegt. Die Interviews wurden zwischen August 2012 und August 2013 durchgeführt. Zumeist wurden die Interviews in den Praxen der entsprechenden Ärzte geführt. Die Doktorandin der vorliegenden Arbeit war an der Interviewerhebung in Göttingen beteiligt.

Vor Beginn eines Interviews wurde darauf geachtet, eine angemessene und angenehme Interviewsituation herzustellen. Anschließend wurden Informationsschreiben und Einverständniserklärung mit dem Arzt durchgegangen und Ziele und Rahmenbedingungen von Interview und Studie nochmals hervorgehoben. Die Ärzte erhielten die Möglichkeit, Fragen zu stellen. Anschließend wurden demografische Daten des Arztes erhoben und das Formular für die Aufwandsentschädigung ausgefüllt. Auf die Wahrung der Datenschutzbestimmungen und der Anonymität wurde nochmals hingewiesen. Die Ärzte wurden darauf hingewiesen, dass ihre ärztliche Schweigepflicht auch für den Rahmen des Interviews greift. Eine Einverständniserklärung wurde jeweils von Arzt und Interviewer unterzeichnet und jeweils ein Exemplar von Arzt und Interviewer einbehalten. Die Interviews wurden per Diktiergerät aufgenommen. Bei der Interviewdurchführung war darauf zu achten, dass der Interviewer flexibel auf die Erzählungen des Interviewpartners einzugehen hat. Die Leitfragen sollten vollständig, aber dennoch an den Gesprächsverlauf angepasst gestellt werden. Immanente (direkt auf das zuvor Erzählte aufbauend) und exmanente (Ansprechen von noch nicht erwähnten Themen) Nachfragen konnten gestellt werden. Zum Abschluss des Interviews erhielten die Interviewpartner die Möglichkeit, für sie relevante Aspekte zu ergänzen. Zur Vervollständigung wurde im Anschluss 
an das Interview ein Memo zum Interview-Setting und zu Beobachtungen vom Interviewer verfasst.

\subsection{Datenanalyse}

Die Interviews wurden wortwörtlich transkribiert und pseudonymisiert sowie nochmals kontrolliert und gegengelesen. Anschließend wurden die Interviews von Mitarbeitern des Projekts nach der Methode der Grounded Theory mittels der Computersoftware MAXQDA (MAXQDA) unabhängig voneinander ausgewertet. Dies geschah anhand des offenen, axialen und selektiven Kodierens nach Strauss und Corbin. Für die ärztlichen Interviews waren die durchführenden Personen: Dr. Jochen Werle, Arbeitsgruppe Psychiatrische Epidemiologie und Demografischer Wandel, Zentralinstitut für Seelische Gesundheit, Medizinische Fakultät Mannheim/Universität Heidelberg, Mannheim sowie Britta Tetzlaff, Institut für Allgemeinmedizin, Universitätsklinikum Eppendorf, Hamburg und Franziska Blank, Doktorandin am Institut für Allgemeinmedizin, Universitätsmedizin Göttingen und Verfasserin dieser Dissertation.

Der Ablauf und die Datenerhebung durch die Doktorandin Franziska Blank, die vorliegende Dissertation betreffend, gestaltete sich wie folgt: Der Einstieg der Doktorandin in das Projekt erfolgte im Winter 2012. Zu Beginn und um sich mit dem methodischen Vorgehen der Interviewanalyse vertraut zu machen, erfolgte eine kurzzeitige Mitarbeit der Doktorandin am Datenmaterial der Interviews von Pflegekräften. Hierbei erfolgte das offene Kodieren und Gegenlesen der Auswertungen in der Gruppe. Zum Zeitpunkt des Einstiegs der Doktorandin in das Projekt lag der Interviewleitfaden für die Pflegekräfte und die Hausärzte bereits ausgearbeitet vor, und es waren bereits einige Interviews durchgeführt worden. Im Weiteren erfolgte dann die Mitarbeit der Doktorandin im Team der die ärztlichen Interviews bearbeitenden Gruppe (Dr. Jochen Werle, Britta Tetzlaff). Der Arbeit dieser Gruppe lag ein anderer Forschungsschwerpunkt zugrunde, als in der vorliegenden Arbeit. F. Blank führte eigenständig vier Interviews am Standort Göttingen, teils in Begleitung des wissenschaftlichen Mitarbeiters Christoph Cavazzini, durch. Für die Gruppe der Hausärzte erfolgte durch F. Blank die Aufstellung der demografischen Daten. Die Doktorandin war erneut am offenen Codieren der Arztinterviews aller Standorte beteiligt. Hierzu wurden die Inter- 
views unter den Teammitgliedern aufgeteilt. Die Doktorandin war wie die anderen Teammitglieder verantwortlich für das eigenständige Erarbeiten und Bilden von Codes und das Gegenlesen von bearbeiteten Arztinterviews ihrer Teammitglieder. Auch beim selektiven Kodieren der Arztinterviews wirkte die Doktorandin zunächst mit. Die Fragestellungen für die vorliegende Promotionsarbeit erarbeitete die Doktorandin selbstständig unter Hilfestellung von den Projektverantwortlichen Prof. Dr. med. E. Hummers und Dr. med. C. Müller. Es folgte dann die erneute Bearbeitung sämtlicher Arztinterviews im Hinblick auf das Promotionsvorhaben durch die Doktorandin. Dies wurde durch die Doktorandin autonom und vollständig durchgeführt. Sämtliche Arztinterviews und bestehende Codes wurden erneut gelesen, bearbeitet und erneut codiert. Um dem Prinzip der Offenheit und Reflexion während dieses Prozesses gerecht zu werden, erfolgten eine enge Absprache und ein stetiger Informationsaustausch mit Dr. med. C. Müller. Für die erste und zweite Fragestellung wurden die im offenen Kodieren erstellten Codes mit entsprechenden Textstellen belegt und anschlieBend thematisch geordnet und inhaltsübergreifend zusammengefasst. Das damit von der Doktorandin erarbeitete Material wurde von dieser mehrfach und in einem sich stetig verdichtenden Prozess bearbeitet und reflektiert. Es erfolgte die Zuordnung und Konkretisierung hinsichtlich der ausgearbeiteten Fragestellungen und schließlich eine schriftliche Aufstellung der erhobenen Ergebnisse. Darüber hinaus führte die Doktorandin neben dem offenen Codieren auch die Schritte des axialen und selektiven Kodierens im Hinblick auf die dritte Fragestellung durch. Hierbei wurde das Kodierparadigma nach Strauss und Corbin von der Doktorandin angewandt. Das zentrale Phänomen der Zerrissenheit wurde von der Doktorandin eigenständig erarbeitet und unter Beteiligung von Dr. med. C. Müller ausgearbeitet. Auch die Schritte des axialen und selektiven Kodierens erfolgten als sich stetig verengender, mehrstufiger Prozess, an dessen Ende die inhaltliche Aufschlüsselung und schriftliche Ausarbeitung der Forschungsergebnisse standen.

In den ausgewählten Interview-Textstellen, welche im Ergebnisteil beispielhaft zitiert werden, wurden zur besseren Lesbarkeit grobe Fehler korrigiert. Es wurde jedoch bewusst auf eine vollständige Korrektur verzichtet, um den Kern der Aussage und das Zitat selbst nicht zu verändern. Vorherige Promotionsarbei- 
ten, welche ebenso das bestehende Interviewmaterial nutzten, wählten ebenfalls dieses Vorgehen.

Das genaue Vorgehen bei der Interviewauswertung wird nun durch die im Folgenden beschriebenen Grundsätze der Analyse nach der Grounded Theory erklärt (Strauss und Corbin 2010) und entspricht der Arbeit in der Gruppe die Gesamtstudie betreffend sowie der Arbeit der Doktorandin, die eigene Promotionsarbeit betreffend.

Die Grounded Theory dient der Erhebung und systematischen Bearbeitung qualitativer Daten. Die Grounded Theory ist eher als Forschungshaltung denn einzelne Methode zu verstehen. Während eines sich verengenden Prozesses werden sämtliche Ergebnisse im Hinblick auf die Forschungsfragen mehrfach reflektiert und miteinander verglichen. Das Vorgehen nach der Grounded Theory dient der Theoriegenerierung. Bei Flick werden diese Theorien als Versionen oder Perspektiven beschrieben, in denen die Welt gesehen wird (Flick 2010). Da den entwickelten Theorien eine realitätsnahe Abbildung sozialer Phänomene anhand wissenschaftlicher Daten zugrunde liegt, steht in erster Linie das Verstehen und nicht das Beschreiben sozialer Phänomene im Vordergrund (Marx und Wollny 2009b; Strauss und Corbin 2010). Die stetige Reflexion des Forschungsvorgehens wird durch das Prinzip der Zirkularität erreicht (Flick 2010; Strauss und Corbin 2010). Hierbei werden die verwendeten Methoden, erstellten Kategorien und Theorien permanent während des Prozesses der Datenerhebung und -auswertung auf ihre Gültigkeit und Sinnhaftigkeit hin überprüft. Auch die eigene Position als Forscher wird reflektiert und bei der Generierung von Theorien bedacht. Der Forscher steht permanent in Interaktion mit den Daten. Somit wird in der Grounded Theory der Forscher nicht getrennt vom Forschungsgegenstand, sondern als ein Teil dessen betrachtet. Der Selbstreflexion dienen auch die vom Forscher während der Datenanalyse verfassten Code-Memos, in denen Eindrücke und Hypothesen zum Datenmaterial festgehalten werden.

Das Vorgehen nach der Grounded Theory sieht drei Kodierschritte vor: das offene, das axiale und das selektive Kodieren. Beim offenen Kodieren werden die Daten „aufgebrochen“, genau untersucht und verglichen. Im Hinblick 
auf die Forschungsfragen - wie beispielsweise die Wahrnehmung von Versorgungsbedürfnissen und -bedarf, Kontinuität der Versorgung, Probleme, Wünsche und Bedürfnisse der Hausärzte - wurden die Interviews Zeile für Zeile durchgegangen und einzelne Wörter, Zeilen, Sätze oder Absätze einem Code zugeordnet. Codes beschreiben den Inhalt einzelner Aussagen bzw. das, wofür diese Aussage steht. Es wurden selbst gebildete Codes oder In-vivo-Codes vergeben. Bei In-vivo-Codes bildet eine Aussage aus dem Interview selbst den Code. Sich ähnelnde Aussagen verschiedener Interviews wurden demselben Code zugeordnet.

Durch Strukturierung der Codes konnten Phänomene benannt und die Codes zu Konzepten geordnet werden. Die Konzepte wurden dann in übergeordnete Kategorien zusammengefasst, wobei eine Kategorie immer ein von den Originaldaten abstrahiertes Konzept darstellt (Marx und Wollny 2009b; Strauss und Corbin 2010). Die Benennungen der Kategorien wurden von den Teammitgliedern verglichen und diskutiert. Vergleichen und Fragen an die Daten stellen war bei dem hier angewendeten Vorgehen grundlegend. Den Kategorien konnten Eigenschaften zugeordnet werden und diese in verschiedene Dimensionen geordnet werden.

Beim axialen Kodieren wurden Kategorien in Verbindung zueinander gebracht mit dem Ziel: Zusammenhänge zu erfassen, Daten zusammenzufassen und somit zu verdichten. Das Ermitteln von Beziehungen zwischen einzelnen Kategorien war ein wichtiger Schritt zur Interpretation der Daten. Wir verwendeten hierfür das Kodierparadigma von Strauss und Corbin (Flick 2010; Strauss und Corbin 2010). Hierbei werden für ein Phänomen dessen Bedingungen und Konsequenzen herausgearbeitet sowie der Kontext und die von den Betroffenen verwendeten Strategien im Umgang mit dem entsprechenden Phänomen.

Beim selektiven Kodieren wurden aus der Vielzahl von Kategorien Kernkategorien ausgewählt und benannt. Die Kernkategorien bilden das zentrale Phänomen in den Daten. Ziel war es, eine verdichtete Theorie zu generieren. Strauss und Corbin empfehlen hierzu, sich den "roten Faden der Geschichte“ deutlich zu machen (Strauss und Corbin 2010, S. 96). 


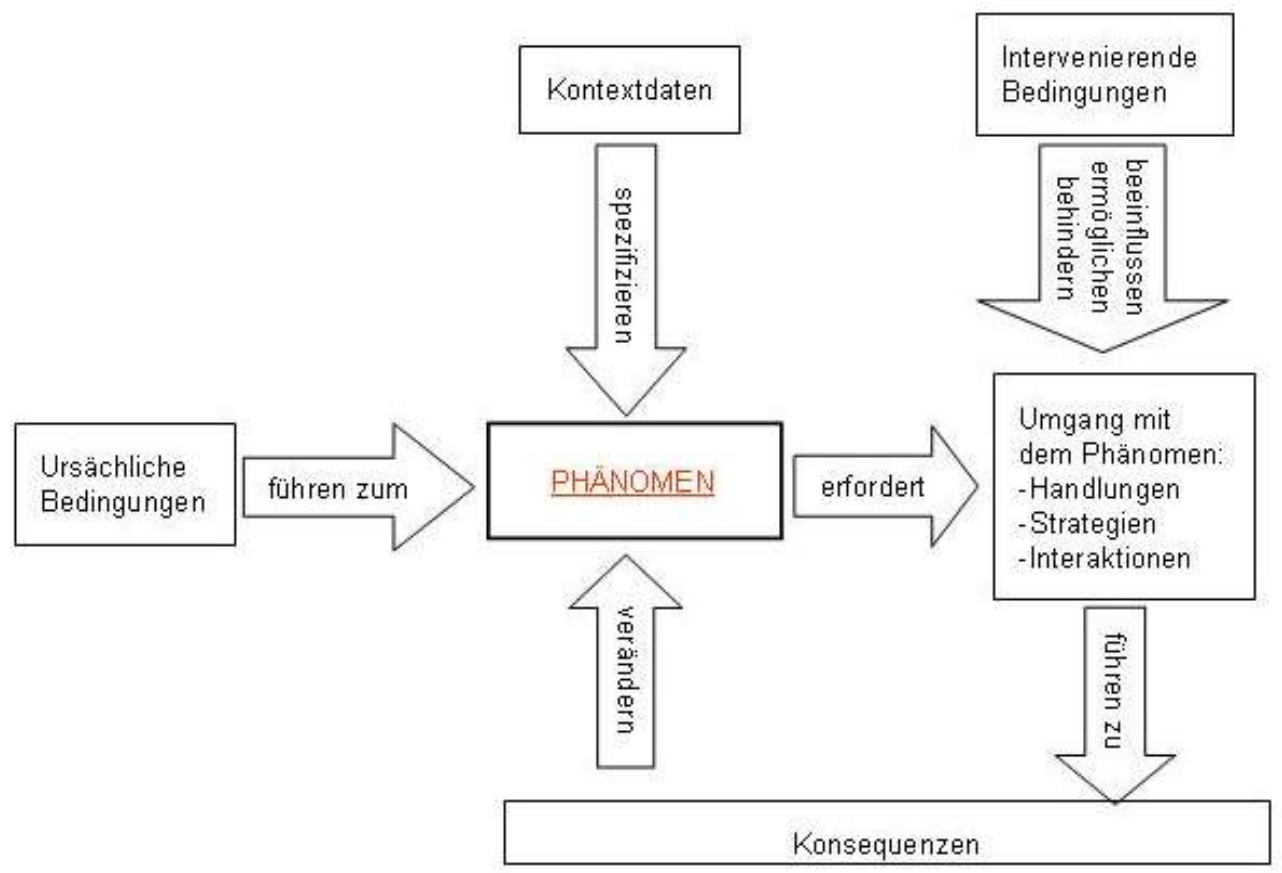

Abbildung 1: Das Kodierparadigma nach Strauss und Corbin aus (Mühlmeyer-Mentzel und Schürmann 2011). Mit freundlicher Genehmigung der Autorinnen.

Das offene Kodieren für das Forschungsvorhaben der INTERPROF-Studie erfolgte als Teamprozess der beteiligten Mitglieder J. Werle, B. Tetzlaff und F. Blank und wurde von F. Blank hinsichtlich der eigenen Fragestellungen erneut bearbeitet und ergänzt. Axiales und selektives Kodieren wurden von F. Blank auf die vorliegenden Fragestellungen hin - alleine und eigenständig durchgeführt.

Zu erwähnen ist, dass die Vorgehensweise nach der Grounded Theory nicht zwangsweise ein schrittweise abzuarbeitendes Schema ist, sondern die einzelnen Kodiervorgänge nebeneinander angewandt werden können. Flick erklärt hierzu, dass die einzelnen Kodierschritte vielmehr verschiedene Umgangsweisen mit dem textuellem Material darstellen würden, zwischen denen der Forscher bei Bedarf hin und her springen und die er miteinander kombinieren könne (Flick 2010). Da die Dissertation einen speziellen Teil der Forschungsfragen hinsichtlich der hausärztlichen Versorgung bearbeitet, wird auf die weiteren Forschungsfragen der gesamten Studie hier nicht weiter eingegangen. 


\subsection{Datenschutz und Genehmigung durch die Ethikkommission}

Die Ethikanträge für die INTERPROF-Studie wurden separat an den beteiligten Instituten der drei Standorte gestellt und durch die dortigen Ethikkommissionen begutachtet und genehmigt. Der von der Ethikkommission der Universitätsmedizin Göttingen genehmigte Ethikantrag für den Standort Göttingen trägt die Nummer 17/5/12. Über dieses Promotionsvorhaben wurde die Ethikkommission der Universitätsmedizin Göttingen in Kenntnis gesetzt. Die Ethikkommission äußerte keine Bedenken hinsichtlich des Dissertationsverfahrens (Antrag DOK_110_2013). 


\section{Ergebnisse}

\subsection{Beschreibung der Stichprobe}

An den drei Standorten wurden jeweils zehn Interviews mit in der Versorgung von Pflegeheimbewohnern tätigen Hausärzten geführt. Es wurden 10 Frauen und 20 Männer befragt. Die Teilnehmer waren zwischen 36 und 71 Jahre alt. Die genaue Verteilung ist in Abbildung 2 dargestellt.

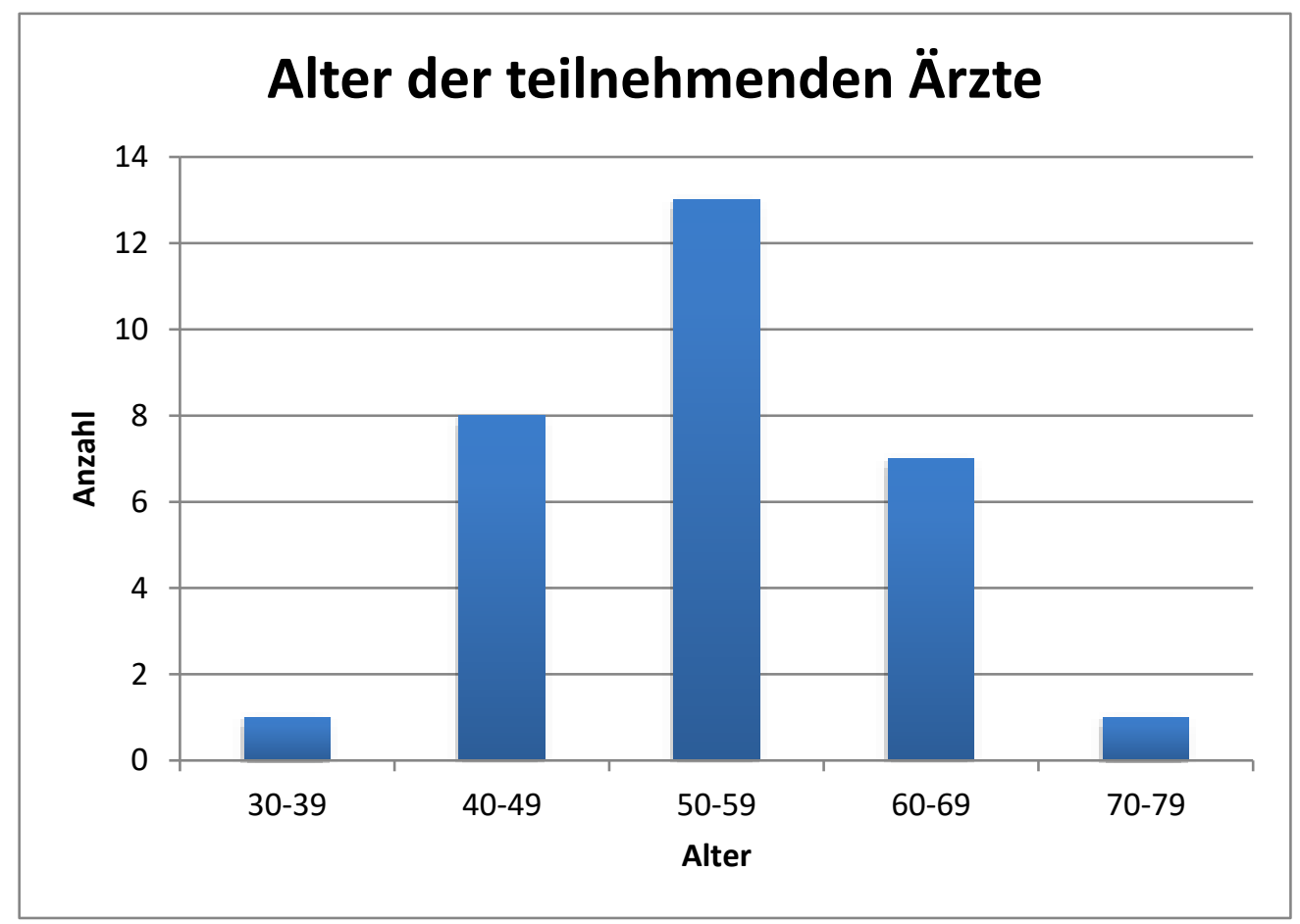

Abbildung 2: Altersverteilung der interviewten Hausärzte

Es wurden Ärzte mit dem Facharzt für Allgemeinmedizin und Ärzte mit dem Facharzt für Innere Medizin interviewt. Einige Ärzte hatten eine weitere Berufsausbildung abgeschlossen (Krankenpflegehelfer, Fotograf, Zahnarzthelferin, Versicherungskaufmann, Bankkauffrau), einige ein weiteres Studium (Health Care Management, Chemie, Public Health, Germanistik). Eine weitere als die derzeitige ärztliche Tätigkeit übten drei der interviewten Ärzte aus, nämlich als wissenschaftlicher Mitarbeiter, als Lehrarzt und Gutachter und als Notdienstkoordinator. 


\begin{tabular}{|l|l|}
\hline Jahre & Anzahl \\
\hline \hline $10-14$ & 4 \\
\hline $15-19$ & 4 \\
\hline $20-24$ & 5 \\
\hline $25-29$ & 4 \\
\hline $30-34$ & 7 \\
\hline $35-39$ & 5 \\
\hline$>40$ & 1 \\
\hline \multicolumn{2}{|l}{} \\
Dauer der ärztlichen \\
Tätigkeit
\end{tabular}

\begin{tabular}{|l|l|}
\hline Jahre & Anzahl \\
\hline$<1$ & 2 \\
\hline $1-5$ & 2 \\
\hline $6-10$ & 5 \\
\hline $11-15$ & 3 \\
\hline $16-20$ & 7 \\
\hline $21-25$ & 6 \\
\hline $26-29$ & 2 \\
\hline $30-35$ & 1 \\
\hline $36-40$ & 1 \\
\hline \multicolumn{2}{|l|}{$\begin{array}{l}\text { Dauer der } \\
\text { Tätigkeit im Heim }\end{array}$} \\
\hline
\end{tabular}

\begin{tabular}{|l|l|}
\hline Stunden & Anzahl \\
\hline $1-5$ & 20 \\
\hline $6-10$ & 8 \\
\hline $11-15$ & 0 \\
\hline $16-20$ & 0 \\
\hline $21-24$ & 0 \\
\hline $25-29$ & 0 \\
\hline$>30$ & 1 \\
\hline \multicolumn{2}{|c|}{} \\
Dauer der & Tätigkeit \\
im Heim pro Woche
\end{tabular}

Tabelle 3: Dauer der ärztlichen Tätigkeit insgesamt, Dauer der ärztlichen Tätigkeit im Heim, Dauer der Tätigkeit im Heim pro Woche

Von den 30 Ärzten arbeiteten 18 in einer Gemeinschaftspraxis, 12 Ärzte waren in einer Einzelpraxis tätig. Alle führten zum Interviewzeitpunkt Hausbesuche im Pflegeheim durch. Die Jahre an Tätigkeit im Heim betrugen drei Monate bis 40 Jahre (siehe Tabelle 3). Die Anzahl der zum Interviewzeitpunkt besuchten Heime variierte bei den einzelnen Ärzten zwischen einem und zehn Heimen. Dort wurden zwischen vier und 250 Patienten betreut. Eine Übersicht über die Verteilung ergibt sich aus Abbildung 3. 


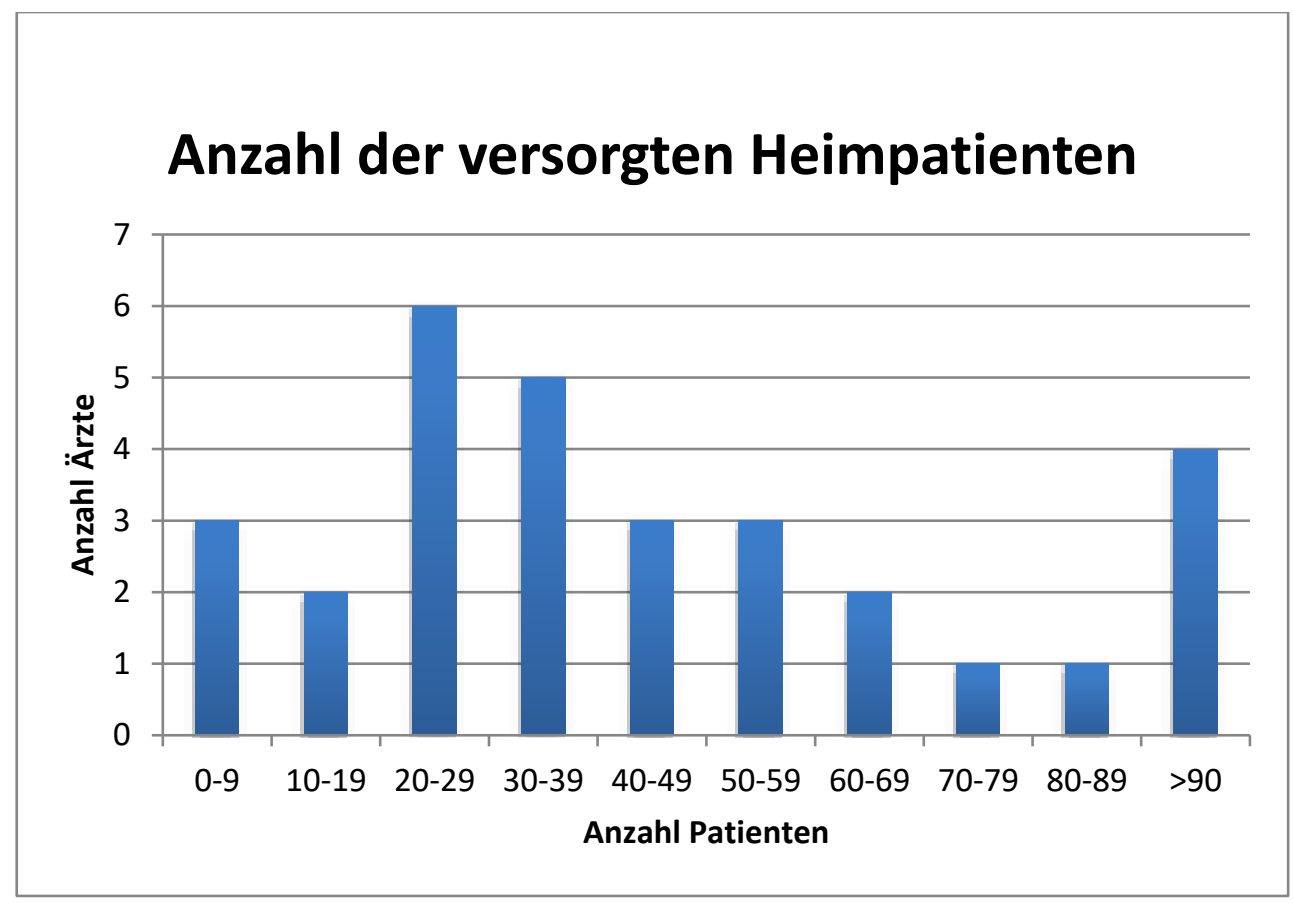

Abbildung 3: Anzahl der versorgten Heimpatienten pro Hausarzt

Für die Versorgung ihrer Heimpatienten brachten die Ärzte zwischen einer und 30 Stunden pro Woche auf. Es gab auch Hausärzte, die nur nach Bedarf und somit unregelmäßig Heimbesuche durchführten.

\subsection{Ergebnisse der Interviewauswertung}

Die Reihenfolge der Darstellung zu den Ergebnissen orientiert sich an den unter 3. beschriebenen Fragestellungen. Jede Frage wurde nochmals in strukturierte Unterpunkte unterteilt. Für Frage eins und zwei wurde ein beschreibendes Vorgehen aus den Ergebnissen des offenen Kodierens gewählt. Für Frage drei folgt eine detailliertere Darstellung nach der Grounded Theory und dem Kodierparadigma nach Strauss und Corbin (Strauss und Corbin 2010). 


\subsubsection{Wahrnehmungen und Bedürfnisse der Hausärzte bezüglich ihrer eigenen Arbeitssituation - Wünsche hinsichtlich einer Lösungsfin- dung}

Die Ergebnisse zu den Wahrnehmungen und Bedürfnissen hinsichtlich der eigenen Arbeitssituation der Hausärzte lassen sich unter vier Teilaspekten zusammenfassen. Diese werden von den externen Rahmenbedingungen, den heiminternen Rahmenbedingungen, der Sicht auf das Thema "Heimarzt“ sowie von allgemeinen Lösungsvorschlägen gebildet und im Folgenden näher erläutert.

\subsubsection{Wahrnehmung der externen Rahmenbedingungen und Bedürfnis- se zu den externen Rahmenbedingungen}

\begin{tabular}{|l|}
\hline Wirtschaftliche Rahmenbedingungen \\
\hline $\begin{array}{l}\text { Die Versorgung von Heimbewohnern als wirtschaftlicher Nachteil bis hin zum } \\
\text { wirtschaftlichen Risiko }\end{array}$ \\
\hline Vergütung beeinflusst Zeit und damit Qualität \\
\hline Wunsch nach besserer Vergütung \\
\hline Gesellschaftliche Rahmenbedingungen \\
\hline Gesetzliche Rahmenbedingungen
\end{tabular}

Abbildung 4: Übersicht zu den externen Rahmenbedingungen

Aus den Interviews ließen sich verschiedene Faktoren herausfiltern, die die Rahmenbedingungen für Hausärzte bilden. Hierbei lassen sich externe von heiminternen Rahmenbedingungen unterscheiden.

Die externen Rahmenbedingungen werden von einer Gruppe von Faktoren gebildet, welche die strukturellen Umstände beinhalten und beschreiben, denen Hausärzte bei ihrer Arbeit im Heim ausgesetzt sind. Hierzu zählen wirtschaftliche, gesellschaftliche und gesetzliche Faktoren. Gemeinsam ist diesen Fakto- 
ren, dass sie von außen auf den Arzt einwirken und dieser in der Regel nur wenig Einflussnahme sieht.

Die wirtschaftlichen Rahmenbedingungen sind ein Thema, das die meisten interviewten Ärzte beschäftigt und das auf die Frage nach den Vorstellungen zur Verbesserung der Arbeitssituation im Heim oft angesprochen wurde. Hierbei sind verschiedene Punkte in den Interviews erkennbar, die dieses Thema so wichtig für die interviewten Ärzte machen. Die Rolle des Hausarztes als Unternehmer wird im Folgenden deutlich:

Die Versorgung von Heimbewohnern als wirtschaftlicher Nachteil bis hin zum wirtschaftlichen Risiko

Die Wahrnehmung der unternehmerischen und wirtschaftlichen Rahmenbedingungen liegt in verschiedenen Ausprägungen vor. Während einige Hausärzte den Heimbesuch als wirtschaftlich ungünstig und als Nachteil beschreiben, steigert sich dies bei anderen bis hin zur Darstellung des Heimbesuchs als wirtschaftliches Risiko für das eigene unternehmerische Fortbestehen und somit zu einer indirekten Existenzbedrohung, die mit der Heimtätigkeit verbunden wird. Andere Hausärzte beschreiben, dass sich der Besuch im Heim finanziell nicht lohnt, er aber zum hausärztlichen Selbstverständnis und Rollenbild gehört oder aus idealistischen Gründen dennoch durchgeführt wird.

„Hier wäre wohl noch zu erwähnen, dass gerade Hausbesuche von dieser rein wirtschaftlichen Seite her gesehen eigentlich das Dümmste ist was man machen kann aber in [...] unseren Weltbildern und in unserem ethischen Selbstverständnis gehört es nun mal dazu und man macht es dann aber es darf keiner davon erwarten, dass man das aus irgendwelchen finanziellen Gründen macht, das würde sich dann wie man so schön sagt lohnen würde sich das überhaupt nicht." (CA09/55)

„...eine gewisse Naivität oder Idealismus oder Dummheit oder was Sie sagen wollen [...] man hat durch diese ganzen Sachen keine ökonomischen Vorteile. " (AA07/140) 
Aus einem anderen Blickwinkel stellt sich der Gesichtspunkt so dar, dass Heimbesuche finanziell ein Minusgeschäft darstellen und deshalb durch den normalen Praxisbetrieb querfinanziert werden müssen. Ein Hausarzt muss demnach überlegen, wie viele Hausbesuche er sich leisten kann, ohne zu groBe Verluste davonzutragen. Zu beachten ist hierbei, dass die genannten Punkte oftmals für Hausbesuche an sich gelten und nicht unbedingt heimspezifisch sind.

„Da muss ich mir immer überlegen, wie viele Hausbesuche kann ich mir eigentlich leisten. Man muss das mal ganz bitter so ausdrücken."(CA07/75)

„...16 Euro für einen Hausbesuch, der mich eine halbe Stunde kostet, das ist absolut kostenundeckend. Das ist für mich ein Risikofaktor, das kostet mich wahrlich Geld, von dem was ich verdiene. Da muss man klar sagen, damit setzt die Politik und die, die Rahmenbedingungen schaffen, setzen ein Zeichen mache nur keinen routinemäßigen Arztbesuch." (CA07/73)

Durch die Heimtätigkeit können für den Hausarzt sogar zusätzliche Kosten entstehen, etwa wenn er wie bei AA02/163-165 beschrieben eine medizinische Fachangestellte als zusätzliche Teilzeitkraft beschäftigen muss, um beispielweise Labor- und Blutentnahmen im Heim durchzuführen. Es bleibt fraglich, in welchem Verhältnis hierbei Ausgaben und Einnahmen durch diese Tätigkeiten stehen.

Hausärzte bemängeln, dass mit dem bestehenden Vergütungssystem falsche und zu wenig Anreize für die Versorgung von Pflegeheimbewohnern gesetzt werden und dass die Bereitschaft zur Heimtätigkeit dadurch sinkt.

„Im Moment würde ich sagen, können sie Ärzte, die Heimpatienten betreuen, mit der Lupe suchen." (CA07/63)

Hausärzte resignieren aufgrund der wirtschaftlichen Rahmenbedingungen. Ihre Erfahrung mit Regressansprüchen lehrt sie, „dass die Einhaltung von Zahlen wichtiger ist als die Behandlung von Menschen" und "dass die Behandlung von 
Altenheimpatienten [...] ein wirtschaftliches Risiko darstellt" (CA07/61). Darüber hinaus führen Regressansprüche und wirtschaftlicher Druck zu einer nicht unerheblichen psychischen Belastung für die Hausärzte.

„...es ist nicht ärztliche Aufgabe rumzusitzen und irgendwelche Regresse zu schreiben, sondern es ist ärztliche Aufgabe Patienten $z u$ betreuen. Abgesehen davon war ich auch unausgeschlafen, weil ich mich ausgesprochen geärgert habe darüber." (AA07/345)

Die Betreuung von Pflegeheimen wird für eine normale hausärztliche Praxis nicht als Praxisbesonderheit gewertet. So können eine Budgetüberschreitung und die damit verbundenen Regressforderungen sich zum größten Teil aus der Versorgung der kostenintensiven Heimpatienten ergeben (CA07/61). Es gibt jedoch auch Hausärzte die beschreiben, dass sich Hausbesuche ab einer gewissen Anzahl sehr wohl lohnen können und in diesem Fall sogar einen erheblichen Anteil am Praxiseinkommen ausmachen.

"ICh will noch nicht verhehlen, dass wir durch diese ungewöhnlich vielen Patienten auch ein besonderes Budget bekommen." (AA03/25)

Die Vergütung der ärztlichen Tätigkeit beeinflusst auch die Zeit, die sich ein Arzt für seine Patienten nimmt und damit nimmt die Vergütung letztendlich auch Einfluss auf die Qualität der ärztlichen Versorgung.

\section{$\underline{\text { Vergütung beeinflusst Zeit und damit Qualität }}$}

Zuwendung und gute, gründliche Versorgung brauchen Zeit (CA06/125). Der Zeitmangel, den die Hausärzte beklagen, wird als Hemmnis für eine gute Versorgungsqualität angesehen (CA07/81). Die Qualität der Versorgung hängt für die Ärzte kausal mit der Vergütung zusammen.

„Und das heißt, wenn eine qualitativ hochwertige Versorgung da sein soll muss die entsprechend vergütet werden, wie immer bei allem." (BA02/119)

Konsequenzen aus den wirtschaftlichen Rahmenbedingungen sind, dass die Bereitschaft, Hausbesuche zu machen, bei den Hausärzten sinkt, und dass das 
ärztliche Handeln an die finanziellen Rahmenbedingungen angepasst wird. Dies widerspricht jedoch dem hausärztlichen Anspruch nach „aufrichtigem“ ärztlichem Handeln. Denn so kommt es vor, dass Routinebesuche als Notfallbesuche deklariert werden, um den Besuch wirtschaftlich zu machen (CA07/75) und besser vergütete Tätigkeiten häufiger durchgeführt werden als schlechter vergütete Tätigkeiten.

\begin{abstract}
„...wenn die Hausbesuche finanziert werden, dann haben alle auf einmal ganz arg viele Hausbesuche. Wenn sie nicht finanziert werden, haben sie auf einmal nur noch Notfallhausbesuche. [...] ich hab keine Lust so ein Spiel mitzumachen [...] man sieht schon, dass das was honoriert wird, wird viel gemacht und was nicht honoriert wird, wird nicht gemacht." (BA04/70)
\end{abstract}

Des Weiteren erfolgt eine Selektion der Patienten nach deren Lukrativität und nach wirtschaftlichen Gesichtspunkten (CA07/65). Der bürokratische Aufwand und die wirtschaftlichen Nachteile durch die Betreuung von Heimpatienten können dazu führen, dass Hausärzte ihre Heimtätigkeit reduzieren oder sogar einstellen. So geschehen bei AA07, der den Aufwand durch schriftlichen Begründungen und Widerspruchseinreichungen bei Regressforderungen beanstandet.

„Jetzt muss ich mich am Sonnabend hinsetzen und den Hausbesuch bei Frau soundso im Pflegeheim begründen. Ja? Gut, dann mache ich das nicht mehr. Dann sollen die doch sehen wer das macht." (AA07/381)

\title{
Wunsch nach besserer Vergütung
}

Die resultierenden Handlungsweisen der Hausärzte aufgrund von wirtschaftlichen Nachteilen können im Umkehrschluss durch finanzielle Anreize und eine angemessene Vergütung positiv beeinflusst werden. Der Wunsch nach einer besseren Vergütung ihrer Tätigkeit ist bei den Hausärzten sehr groß, zum einen, um ihr „mittelständisches Unternehmen Arztpraxis“ (CA03/52) am Laufen halten können, zum anderen, um sich mehr Zeit für den einzelnen Patienten im Heim nehmen zu können. 
„Also ich würde mir wünschen, dass es möglich wäre, dass das ganze so gut honoriert ist, dass man zum Beispiel sich auf ein Pflegeheim konzentrieren könnte und dafür auch die Zeit hätte. [...] dass man so normal daran auch verdienen könnte, dass man sich die Zeit nehmen könnte." (BA05/169)

Den Wunsch nach einer besseren Vergütung der Hausbesuchstätigkeit im Heim begründen Hausärzte mit den folgenden Argumenten:

- Heimbewohner sind multimorbide und haben dadurch einen höheren Versorgungsaufwand. Das Alter der Heimpatienten steigt immer weiter an und verschärft dieses Problem. Dies müsste in der zeitlichen und damit auch in der finanziellen Planung berücksichtigt werden (CA10/34).

- Hausbesuche sind anstrengend. Die Praxistätigkeit wird als weniger erschöpfend und besser vergütet beschrieben (CA08/119).

- Hausbesuche sind zeitaufwendig. Allein die langen und schlecht vergüteten Anfahrten fallen hierbei ins Gewicht (BA06/112) (AA06/42).

Als Lösung für den bestehenden ökonomischen Konflikt wünschen sich die Hausärzte unter anderem eine Abschaffung des Regresssystems (CA07/65), ein höheres Budget für die Betreuung von Heimpatienten (BA07/119) und eine Zunahme der gesellschaftlichen Wertschätzung ihrer Arbeit. Diese Wertschätzung ist Teil der gesellschaftlichen Rahmenbedingungen und scheint für die Hausärzte auch stark mit einer angemessenen Vergütung zusammen zu hängen (CA08/121). Bezüglich der gesellschaftlichen Rahmenbedingungen wird des Weiteren als Lösungsansatz geäußert, dass sich das Anspruchsdenken von Patienten ändern müsse. Hausärzte wünschen sich, dass mehr Patienten ihre Praxis aufsuchen und sie somit generell weniger Hausbesuche leisten müssten (CAO3/56). Hier äußert sich auch das Problem des demografischen Wandels, welcher zu einem steigenden Bedarf an ärztlicher Versorgung führt. Um diesen zu decken, wünschen sich Ärzte $u$. a. eine Übernahme von ärztlichen Tätigkeiten durch Pflegekräfte (CA03/56). Hier ist jedoch zu betonen, dass es auch einige Hausärzte gibt, die einer solchen Übernahme von ärztli- 
chen Tätigkeiten durch die Pflegekräfte kritisch gegenüber stehen (CA08/7679) (CA10/118-120).

Grundsätzlich bleibt zu erwähnen, dass es hinsichtlich der wirtschaftlichen und gesellschaftlichen Rahmenbedingungen und der Kritik der Hausärzte an diesen etliche Überschneidungen zwischen Hausbesuchen an sich und Heimbesuchen im Speziellen gibt.

$\mathrm{Zu}$ den gesetzlichen Rahmenbedingungen äußern sich Hausärzte besonders zu ihren Vorstellungen hinsichtlich der Dokumentationsaufgaben. Hausärzte kommen zumeist dem vom Heim geäußerten Dokumentationswunsch der aufgrund von Vorgaben durch den Medizinischen Dienst der Krankenkassen besteht - nach, obwohl die Ärzte selbst durch die Kassenärztliche Vereinigung von diesem Umfang der Dokumentationspflicht entbunden sind (so von innen beschrieben). Die Dokumentation erfolgt als Entgegenkommen dem Heim gegenüber und weil Hausärzte die dadurch entstehende beiderseitige Verbindlichkeit schätzen (CA03/26) (CA05/64). Trotzdem wünschen sich die Hausärzte insgesamt eine Entlastung von bürokratischen und dokumentarischen Aufgaben, nicht zuletzt deshalb, weil es der Versorgung der Patienten nicht zu Gute komme.

„Also Dokumentation in dem Umfang wie im Moment gefordert wird vom Gesetzgeber ist schwachsinnig. Weil es nix bringt. Es hilft dem Patienten nicht weiter." (BA02/101)

Zusammenfassend ist festzustellen, dass bei den externen Rahmenbedingungen besonders die wirtschaftlichen Bedingungen für die interviewten Hausärzte im Vordergrund stehen. Für die Hausärzte überwiegen die Nachteile und Missstände auf diesem Gebiet. Der wahrgenommene wirtschaftliche Nachteil, der Druck durch mögliche Regressforderungen und die ungünstigen Auswirkungen auf Zeit und Qualität der Patientenversorgung lassen eine Vielzahl der Hausärzte die Heimtätigkeit mit negativen Emotionen verbinden und verhindern die Erfüllung von hausärztlichen Bedürfnissen. Es entsteht der deutliche Wunsch nach einer besseren Vergütung. Eine bessere finanzielle und gesellschaftliche Wertschätzung sowie die Entlastung von Dokumentationspflichten und damit 
die Möglichkeit der Fokussierung auf das ärztliche Handeln können als Kernbedürfnisse aus diesem Ergebnisteil abgeleitet werden.

\subsubsection{Wahrnehmung der heiminternen Rahmenbedingungen und Be- dürfnisse zu den heiminternen Rahmenbedingungen}

Heiminterne Rahmenbedingungen und Strukturen beinhalten eine Reihe von Faktoren, welche die hausärztliche Arbeit und Versorgung von Patienten im Heim beeinflussen. Sie entstammen den Gegebenheiten im Heim und betreffen die Zusammenarbeit mit den Pflegekräften und der Heimleitung und die Versorgung von Bewohnern durch die Pflegekräfte. Die Zusammenarbeit mit den Pflegekräften und der Heimleitung trägt wesentlich zur Wahrnehmung und Bewertung der Arbeitsbedingungen durch die Hausärzte bei. Hierbei gibt es sowohl unterstützende und die Arbeitssituation erleichternde Faktoren als auch Erschwernisse der Arbeitssituation durch die Zusammenarbeit.

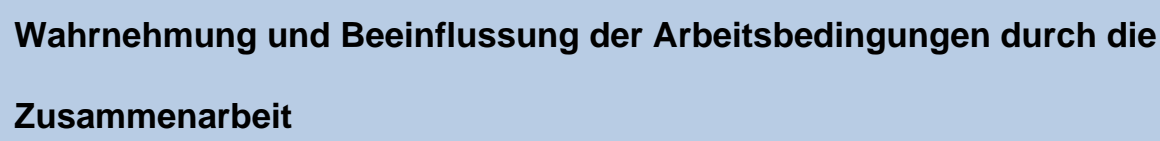

Erkennen und Mitteilen von Auffälligkeiten und Initiierung von Heimbesuchen

Blick der Hausärzte auf die Zusammenarbeit beim Heimbesuch

Entlastung durch Übernahme von Kommunikationsaufgaben

Erreichbarkeit der Pflegekräfte

Bedürfnisse und Wünsche hinsichtlich der Zusammenarbeit

„An einem Strang ziehen“

Treffen von Entscheidungen und Informationsaustausch

Beschwerdekultur

Wunsch nach interprofessionellen Fallbesprechungen

Bedürfnisse und Vorstellungen zu heiminternen Rahmenbedingungen

Nutzung von Diagnostik und medizinischen Verbrauchsmaterialien

Abbildung 5: Übersicht zu den heiminternen Rahmenbedingungen 


\section{Wahrnehmung und Beeinflussung der Arbeitsbedingungen durch die Zu- sammenarbeit}

Hausärzte erfahren in ihrer Tätigkeit dadurch Unterstützung, dass erfahrene Pflegekräfte die Alltagskompetenzen und den mentalen Zustand der Bewohner besser im Blick haben als der Arzt. Auch Vitalparameter lassen sich durch den Arzt schnell und zuverlässig bei den Pflegekräften erfragen. Durch präzise Informationsweitergabe auf diesen Gebieten erhält der Hausarzt ein vollständiges Bild der Bewohner. Dies ist bei Behandlungsplanungen und Therapien sinnvoll. Probleme können so besser detektiert werden (CA01/10) (AA09/113).

Hausärzte schätzen eine gute Dokumentationskultur und Organisation. Dazu gehören gut gepflegte Patientenkurven und feste Abläufe und Strukturen, z. B. beim Hausbesuch. Hausärzte empfinden es als sinnvoll, wenn eine zentrale Verwaltungsstelle z. B. zum Einlesen der Krankenkassenkarten im Heim besteht. Dies führt zu einer Zeitersparnis, da ein einzelnes Erfragen und Einlesen der Karten auf den verschiedenen Wohnbereichen entfällt.

„In dem Heim wo ich eigentlich sehr gerne hingehe, da sind die Pflegechefs sozusagen auf den Stationen, die Wohnbereichsleiter, sehr organisiert. Die Kurve [...] da weiß man sofort wo man hingreifen muss, es ist gut übersichtlich geschrieben." (BA03/46)

„...habe ich ein Heim wo das so läuft, dass ich gerne hingehe. Das geht schon damit los, dass es einen zentralen Ort gibt, wo alle Krankenkassenkärtchen und alle Befreiungsausweise zusammenlaufen und das heißt mein Weg dort rein ist im Prinzip zuerst in diese Verwaltung, da sitzt immer jemand [...] ohne das ich, wie das in allen anderen Heimen ist, von Station zu Station rennen muss, jemanden suchen muss der mir den Schrank aufsperrt, der dann die Visit- diese Kärtchen rausgibt. Also da bleibt, da geht schon ganz viel Zeit." (BA03/118) 
Da der Hausarzt selbst oft nicht vor Ort sein kann, und die Bewohner sich teilweise bei gesundheitlichen Veränderungen nicht mehr selbstständig beim Hausarzt melden können, ist eine aktive Wahrnehmung und Kommunikation über den Gesundheitszustand der Bewohner seitens der Pflegekräfte von Nöten. Dies bedarf einer aufmerksamen und qualifizierten Pflegekraft. Hausärzte profitieren sehr von diesem Informationsgewinn durch die Pflegekräfte und sind dankbar, wenn dies gut funktioniert.

„...die sehen die Bewohner tagtäglich, ich komme nur sporadisch vorbei und dann meistens nur für wenige Minuten [...] bin ich froh, dass ich die habe." (CA04/112)

„Ich kriege einfach Informationen, die ich von den Patienten alleine nicht kriegen könnte. "(AA08/90)

Hausärzte wünschen sich, ihre Patienten gut und bedarfsgerecht zu versorgen. Hierfür sind sie auf die Hilfe und Mitarbeit der Pflegekräfte angewiesen, die ein wichtiges Bindeglied in der Kommunikationskette zwischen Patient und Hausarzt darstellen. Die Erwartung der Hausärzte ist, Auffälligkeiten und Besonderheiten hinsichtlich des Gesundheitszustandes der Bewohner von den Pflegekräften mitgeteilt zu bekommen.

„Meine Erwartung ist, dass sie die Patienten oder die Bewohner wahrnehmen, dass sie merken wenn sie sich verändern oder irgendeine Erkrankung im Anzug ist oder verhindern, dass sie krank werden, sprich mit Dekubitus. " (BA04/154)

Die zeitliche und persönliche Nähe der Pflegekräfte zu den Bewohnern kann dabei sehr zum Vorteil des Hausarztes sein. Hierbei wird immer wieder die Schmerz- und Wundversorgung erwähnt sowie die Betreuung von dementen Bewohnern. In diesen Bereichen wird den Pflegekräften oftmals besondere Kompetenz zugesprochen.

Der Hausarzt ist bei seiner medizinischen Versorgung auf die Pflegekräfte an- 
gewiesen, um aktiv werden zu können. Klappt dies gut, also werden die Ärzte vom Heim kontaktiert, wenn sich Auffälligkeiten oder Veränderungen ergeben, oder wird der Hausarzt bei der Visite im Heim von den Pflegekräften auf Besonderheiten und Veränderungen des Gesundheitszustandes hingewiesen, trägt dies zu einer positiven Beeinflussung der Arbeitswahrnehmung bei. Hierzu ist es von Nöten, dass die Pflegekräfte kompetent und differenziert Gesundheitszustände einschätzen können und entscheiden können, wann der Hausarzt gebraucht wird. Informationen und Visitenanforderungen bei jeglicher Gesundheitsbeeinträchtigung, beispielsweise einer im Heim gut handhabbaren Erkältung eines Bewohners, empfinden Hausärzte als äußerst unbefriedigend.

„Und schön ist auch, wenn sie mich nicht irgendwie für so jeden Nieser einsetzen, sondern dann, wenn die Leute krank sind oder wenn sie sagen: „Der ist erkältet", mir sagen können, ob derjenige Fieber hat, ob es ihm schlecht geht. Wie lange das geht. Also so die Basisanamnese." (AA08/56)

Mehrere Hausärzte sehen hier Verbesserungspotenzial. Es wird erwünscht, dass die Pflegekräfte selbstständiger und aktiver eine solche Kommunikation über den Verlauf bei einem Bewohner gestalten und mehr „medizinischen Sachverstand" aufweisen (AA08/56). Hausärzte verlassen sich in der Regel darauf, dass sich die Pflegekräfte bei Auffälligkeiten melden würden (AA10/118).

Ein Marker für die Qualität der Zusammenarbeit ist für viele Hausärzte insbesondere die Kommunikationskette und das Verhalten von Pflegekräften in Akutsituationen. Hausärzte erachten die richtige Einschätzung von Gesundheitszuständen durch die Pflegekraft als wichtige Kernkompetenz.

"Wie die reagieren auf Akutfälle, wann ich informiert werde, wie ich informiert werde, wie ich sag jetzt mal kompetent auch die Aussage desjenigen ist, ob er Deutsch spricht auch ein Kriterium." (BA04/96)

Hierbei kommt es oft zu Frustration beim Hausarzt, etwa wenn Gesundheitszustände falsch oder unzureichend erkannt werden. Viele Hausärzte bemängeln 
die Kompetenzen der Pflegekräfte in diesem Bereich. Die mangelnde Kompetenz führt wiederum dazu, dass der Hausarzt stärker frequentiert wird.

Oftmals sind Hausärzte über eine mangelnde Informationskultur enttäuscht. Von vielen Hausärzten wird hier ein Nicht-Informieren über Krankenhauseinweisungen ihrer Patienten genannt. Auch das Nicht-Informieren darüber, dass ein Bewohner und Patient des Hausarztes verstorben ist, wird mit großem Unmut berichtet. Besonders, da dies den Hausarzt auch bei Angehörigen in prekäre Situationen bringen kann. Es wird erwähnt, dass eine gute Pflegekraft den Kontakt zu Patient und Hausarzt suchen sollte.

,...einer den ich auch speziell besuchen wollte, hat sich herausgestellt war im Krankenhaus, ohne dass mir das Heim das mitgeteilt hatte. Habe ich natürlich gleich meinen Unmut bekundet, dass ich so was wissen möchte." (CA04/34)

„Eine gute Pflegekraft [...] sucht neben dem Kontakt zum Patienten auch den Kontakt zu mir als Hausarzt [...] Ein Beispiel: Ich traf auf dem Markt eine Angehörige eines Patienten und sagte: "Ich habe ihren Mann vor vierzehn Tagen zuletzt gesehen. Er hatte sich doch erfreulich gebessert." Die mir sagte, dass er [...] am Freitag beerdigt worden wäre. Das war mir verständlicherweise peinlich, nicht?" (AA07/42)

Faktoren mit denen ein Hausarzt bei seiner Arbeit im Heim konfrontiert wird und die sich ungünstig auf die Wahrnehmung der Arbeitsbedingungen auswirken können, sind die ungünstigen Unterbrechungen der Praxissprechstunde durch Heimnotfälle und die problematische und aufwendige Auseinandersetzung mit externen Dienstleistern, wie z. B. Lieferanten von Sondenkost oder auch die Zusammenarbeit mit vom Heim bestellten Wundmanagern (BA09/82). Diese Faktoren führen dazu, dass der Hausarzt auch in seiner Praxistätigkeit negativ durch die Arbeit im Heim beeinflusst wird.

„Na Notfallbesuch würde man jetzt ja noch unterscheiden, entweder das Heim ruft an und sagt jetzt „Herr XY bekommt schlecht Luft oder hat seit heut Morgen 40 Grad Fieber, können Sie sofort 
mal vorbeikommen." Das wäre ja sozusagen für einen Hausarzt das Ungünstigste, die ungünstigste Variante, weil er dann die ganze Sprechstunde in der Praxis liegen lassen muss." (CA03/04)

\section{Blick der Hausärzte auf die Zusammenarbeit beim Heimbesuch}

Hausärzte schätzen es, wenn von den Pflegekräften Fragen ihrerseits zu den Bewohnern gesammelt werden und der Hausarzt im Heim erwartet wird (AA01/03). Wenn die Bewohner auf den bevorstehenden Arztbesuch vorbereitet sind, wird das ebenfalls als positive Arbeitssituation wahrgenommen. Diese Vorbereitung umfasst die Information der Bewohner über den bevorstehenden Arztbesuch, eine Koordination in dem Sinn, dass die Bewohner in ihren Zimmern anzutreffen sind oder auch, dass eventuell Verbände schon abgenommen wurden. Darüber hinaus schätzen es Hausärzte, wenn Patientendokumente gerichtet sind und vorliegen (BA10/83). Über die Begleitung der Pflegekräfte beim Besuch herrscht Uneinigkeit bei den Hausärzten. Viele befürworten eine Begleitung und hierbei besonders die Hilfe der Pflegekräfte, zum Beispiel beim Entkleiden der Bewohner, oder auch den unmittelbaren Informationsgewinn durch die Pflegekräfte. Andere dagegen sehen den Besuch, ähnlich wie die Konsultation in der Hausarztpraxis, als private Situation zwischen Arzt und Patient, in der dem Patienten auch die Möglichkeit eingeräumt werden soll, Dinge anzusprechen, die er vor den Pflegekräften nicht äußern möchte. Auch sehen einige Hausärzte keinen Zeitgewinn durch die Begleitung einer Pflegekraft. Indem sie ihren eigenen Rhythmus bei den Heimbesuchen umsetzen können, fühlen sie sich alleine wohler und auch effizienter. Durch die Praxistätigkeit sind die Hausärzte das selbstständige und autonome Arbeiten darüber hinaus gewohnt.

Durch eine Vorauswahl und Vorabplanung des Besuchsablaufs können die Pflegekräfte den Hausärzten Zeit ersparen. BA06 beschreibt dies als eine Art „Filter“. Die Pflegekräfte überlegen im Vorfeld, welcher Bewohner wie dringend einen Arztbesuch benötigt, und schaffen durch diese Priorisierung einen strukturierten Besuchsplan.

„Sicherlich ist da der Filter vorher, nämlich die Pflege, die mir sagt 
„okay bei inm brennt es, da ist es in Ordnung, da brauchen wir nicht vorbei, hier reicht es auch, wenn sie nächste Woche mal schauen." [...] dann ist es ist es sicherlich schon ein bisschen angenehmer." (BA06/98)

Auch in der Nachbereitung von Heimbesuchen können die Hausärzte Unterstützung durch die Pflegekräfte erfahren. Erhalten die Ärzte eine Rückmeldung über das Anschlagen neu angesetzter Medikamente, erleichtert das die Arbeit der Ärzte. Zur Therapiekontrolle müssen sie weniger häufig in die Heime fahren (CA03/34). Es wird jedoch auch bemängelt, dass diese Rückmeldungen oft unzureichend seien (CA08/34).

\section{Entlastung durch Übernahme von Kommunikationsaufgaben}

Durch die Übernahme von Kommunikation und Klärung von Therapiemaßnahmen mit Angehörigen oder Betreuern kann dem Hausarzt von Seiten des Pflegeheims Arbeitsentlastung verschafft werden.

„Sie nehmen mir ja auch manchmal Arbeit ab, zum Beispiel wenn die Frage ist: Wer kriegt eine Grippeimpfung? Dann klären die für mich auch mit den gesetzlichen Betreuern, wer eine Grippeimpfung haben soll. Also das ist so ein Beispiel, wie sie mir auch Arbeit abnehmen." (AA08/90)

\section{Erreichbarkeit der Pflegekräfte}

Von den Hausärzten oft angesprochen wird das Problem der Erreichbarkeit der Pflegekräfte. Viele Hausärzte bemängeln, dass sie sich die Pflegekräfte beim Heimbesuch erst "herbeiklingeln“ müssten. Dies oder auch das aktive Suchen nach einer (zuständigen) Pflegekraft stellen eine Zeitverzögerung und damit eine Beeinträchtigung des ärztlichen Arbeitsablaufes dar (CA03/22) (CA07/12). Hausärzte erkennen die oft bestehende Unterbesetzung der Pflegekräfte als Hauptursache dieses Problems an (CA06/02) (CA10/52). Sie äußern sich positiv, wenn Pflegekräfte zur Minderung dieses Problems Hilfsmittel wie Lampen über den Türen oder Schilder nutzen, die auf ihren derzeitigen Aufenthaltsort aufmerksam machen (CA05/48). Eine stärkere Inanspruchnahme solcher Maß- 
nahmen scheint aus Hausarztsicht wünschenswert, kann einen grundsätzlichen personellen Mangel jedoch nicht kompensieren.

Die zeitliche Arbeitsbelastung eines Hausarztes wird durch die Arbeit und Kompetenz der Pflegekräfte beeinflusst. Je nach Ausbildungsstand der Pflegekräfte kann dies zum Positiven oder zum Negativen tendieren.

„Und ja das ist so von Heim zu Heim einfach verschieden zeitaufwendig, je nachdem wie gut, wie gut ausgebildet auch die Krankenpfleger sind oder die Altenpfleger die dort in den Heimen sind." (BA03/10)

\section{Bedürfnisse und Wünsche hinsichtlich der Zusammenarbeit}

Hausärzte äußern eine Reihe von Bedürfnissen und Wünschen, welche die Zusammenarbeit mit den Pflegekräften betreffen.

\section{„An einem Strang ziehen“}

Hinsichtlich der Versorgung und Betreuung der Bewohner formulieren die Hausärzte meist klare Vorstellungen und Wünsche. Die Zusammenarbeit betreffend wird der Wunsch geäußert, Pflegekräfte und Hausärzte sollen gemeinsam zum Wohle des Patienten agieren und „an einem Strang ziehen.“

„Also ich würde mir wirklich wünschen, dass es für jeden selbstverständlich ist, dass man so zum Wohl des Patienten an einem Strang zieht, so dass das im Vordergrund steht und nicht irgendwelche Protokolle oder dieses oder jenes oder Reglements." (CA05/145)

\section{$\underline{\text { Treffen von Entscheidungen und Informationsaustausch }}$}

Einige Hausärzte äußern das Bedürfnis nach einer gemeinsamen Entscheidungsfindung mit den Pflegekräften und einem gegenseitigen Informationsaustausch.

"Ich habe manchmal Informationen, die die nicht haben und die haben Informationen, die ich nicht habe. Dass man schon irgend- 
wie versucht da zusammen das Beste rauszufinden." (AA08/126)

\section{Beschwerdekultur}

Hausärzte erleben es oft, dass Kritik zu persönlich genommen wird und keine konstruktive Beschwerdekultur herrscht. Sie äußern daher den Wunsch, nach Etablierung einer guten Beschwerdekultur und einer Kommunikation, besonders Probleme und Kritikpunkte betreffend, auf Augenhöhe. Als hilfreich würden sie hier die Einrichtung einer regelmäßigen Supervision empfinden.

„ICh finde, es sollte eine Möglichkeit geben sich kultiviert über "Nicht-Gutes" zu unterhalten...Beschwerdekultur, so etwas. [...] Nicht vorwurfsvoll, sondern konstruktiv [...] ich würde mich freuen, wenn jeder so selbstbewusst ist, dass er ohne Affekt dem anderen [...] dann sagt: "Das hat mich jetzt da so und so betroffen gemacht - ich würde Ihnen das einfach gerne mal sagen." [...] So was fände ich gut." (AA02/196-198)

„...und da haben wir so eine Supervisionsrunde gemacht, weil da eben auch bei den Pflegekräften [...] dann eben doch erhebliche Konflikte sich ergeben [...]. Wenn Konflikte aufgelöst werden können, das ist gut." (AA07/241)

Gerade bei kritischen Auseinandersetzungen wünschen sich jedoch ein paar Hausärzte, dass eine heiminterne Hierarchie bestehen sollte. Es wird ein Vergleich zu Krankenhausstrukturen gezogen, wo Probleme auch oftmals auf höherer Ebene diskutiert werden. Es besteht also der Wunsch, nach einem koordinierten und konstruktiven Vorgehen.

„Und ich sage, im Krankenhaus kann auch nicht jeder, jede Lernschwester im ersten Lehrjahr gleich zu mir als Arzt rennen und sagen „sie müssen mal nach dem Patienten gucken“, auch im Krankenhaus ist das ähnlich reguliert." (CA07/101)

Zu einer guten Kommunikationskultur gehört auch, sich in positiven Fällen gegenseitig Anerkennung kommunizieren zu können. 
„Wenn wir uns gegenseitig unsere Anerkennung kommunizieren könnten. Das würde ich mir wünschen. [...] Ich glaube, das würde es unheimlich geschmeidig machen." (AA02/186-192)

\section{Wunsch nach interprofessionellen Fallbesprechungen}

Ein weiterer, auch die Kommunikation betreffender Punkt, ist der Wunsch nach regelmäßigen patientenbezogenen Konferenzen und Fallbesprechungen im Team und nach den entsprechenden zeitlichen Ressourcen hierfür (AA07/211). Das positive Erleben der Arbeit im Heim durch feste pflegerische Ansprechpartner spiegelt sich in dem Wunsch wider, diese Kontinuität in der Zusammenarbeit weiter zu fördern. Als möglichen Lösungsvorschlag bringen einige Hausärzte die Idee eines gleich bleibenden, kompetenten Ansprechpartners an, der schichtübergreifend arbeiten könnte und somit das Problem des Informationsverlustes zwischen den einzelnen Schichten minimiert werden könnte (CA03/42) (CA04/78).

\section{Bedürfnisse und Vorstellungen zu heiminternen Rahmenbedingungen}

\section{Nutzung von Diagnostik und medizinischen Verbrauchsmaterialien}

Als einschränkend und verbesserungswürdig wird von vielen Hausärzten empfunden, dass diagnostische Möglichkeiten und Verbrauchsmaterialien im Heim oft nicht zur Verfügung stehen und vom Hausarzt zum Besuch mitgebracht werden müssen. Hausärzte fassen hierunter verschiedene Dinge zusammen, nämlich zum einen diagnostische Möglichkeiten wie Ultraschall- oder EKGGeräte oder auch Material, um einen diagnostischen Abstrich vornehmen zu können, zum anderen Verbrauchsmaterialien wie Verbandsmaterial oder Nahtmaterial (CA01/10) (BA06/82) (BA09/26). Allerdings gibt es auch Hausärzte, die diese Art des Arbeitens als Abwechslung zu ihrer Praxistätigkeit ohne einen "Gerätepark" an diagnostischen Möglichkeiten schätzen (AA06/150). Hausärzte können hierin eine spannende Herausforderung sehen. Es soll Erwähnung finden, dass bei Veränderungen bezüglich dieses Punktes besonders der Kostenträger involviert werden müsste. Ähnlich den Hausärzten, die ein Heimarztkonzept befürworten, wird oft das Bedürfnis nach einem Untersuchungszimmer mit der entsprechenden Ausstattung im Heim geäußert 
(CA04/90). Dieser eine Raum könnte von mehreren im Heim tätigen Ärzten wechselseitig benutzt werden (BA02/97). Ein weiterer Vorteil solch eines Untersuchungszimmers wäre die Stärkung der Privatsphäre der Bewohner und mehr Ruhe für und während der Untersuchung und des Gesprächs (AA10/154). Auch bei diesem Aspekt bleibt zu erwähnen, dass es durchaus auch Hausärzte gibt die es schätzen, dass diese Strukturen im Heim nicht vorliegen. Sie sehen dies als Abwechslung zum Praxisalltag und genießen das „in Bewegung sein“ beim Heimbesuch von Zimmer zu Zimmer (AA01/153).

Als weiteres Bedürfnis wird die Verfügbarkeit von elektronischen und aktualisierten Kommunikationsplattformen genannt. So wird der Wunsch nach elektronischem Zugriff auf die heiminterne Patientendokumentation von der Hausarztpraxis aus bzw. der Ausbau eines besehenden online-Zugriffes geäußert (CA01/28) (BA04/142-143). Es soll hier Erwähnung finden, dass bei der Umsetzung solcher Wünsche die Prüfung der Legalität und die Wahrung des Datenschutzes im Vordergrund stehen müssen.

Es gibt Hausärzte, die erklären, dass eine Verbesserung ihrer Arbeitssituation und ein stärkeres (zeitliches) Engagement ihrerseits unter den bestehenden Rahmenbedingungen im Heim nicht realisierbar scheinen.

„...die momentanen Kriterien lassen da nicht mehr zu. Also ich denke, das muss man schon klar sagen, dass man die Rahmenbedingungen in den Altenheimen verbessern muss, wenn man eine bessere Leistung haben will." (CA07/118)

Zusammenfassend wird klar, dass besonders die Zusammenarbeit mit den Pflegekräften und der Heimleitung die Sicht der Hausärzte auf die Arbeit im Heim bildet und beeinflusst. Hierbei erfahren die Hausärzte in zahlreichen Punkten Unterstützung durch die Zusammenarbeit, sei es während des Heimbesuchs selbst oder schon im Vorfeld bei der Initiierung und Klärung der Notwendigkeit eines Besuchs. Aus den Interviews lassen sich jedoch auch eine Vielzahl von Faktoren hinsichtlich der Zusammenarbeit ableiten, die zu verbessern wären. Auch diese betreffen besonders die Zusammenarbeit und beinhalten wichtige Punkte wie den Ausbau von gegenseitigem Informationsaus- 
tausch, die Etablierung einer Beschwerdekultur oder den Wunsch nach gemeinsamen Fallbesprechungen. Die Verbesserung von strukturellen Dokumentationsgegebenheiten wird darüber hinaus als wichtiger Punkt angesehen.

\subsubsection{Sicht der Hausärzte auf das Thema „Heimarzt“}

$$
\begin{aligned}
& \text { Vorstellungen zur Gestaltung eines Heimarztkonzepts } \\
& \hline \text { Vorteile eines Heimarztkonzepts für den Hausarzt } \\
& \hline \text { Problematiken eines Heimarztkonzepts für den Hausarzt }
\end{aligned}
$$

Abbildung 6: Übersicht zum Thema „Heimarzt”

In den Interviews kamen viele der teilnehmenden Hausärzte auf das Modell eines „Heimarztes“" zu sprechen. Da es für viele Ärzte ein zentrales Zukunftskonzept darzustellen scheint, soll auf dieses Thema hier gesondert eingegangen werden. Die Vorstellungen, was genau unter einem "Heimarzt" verstanden wird, gehen bei den interviewten Ärzten auseinander und werden im Folgenden näher erläutert. Auch Stimmen gegen ein Heimarztkonzept kommen zum Tragen. Einigkeit besteht jedoch darin, dass sich die ein Heimarztkonzept befürwortenden Ärzte eine Zentralisierung und stärkere Kontinuität in der Versorgung im Heim wünschen.

\section{Vorstellungen zur Gestaltung eines Heimarztkonzepts}

Wie ein Heimarztkonzept aussehen könnte, dazu gibt es unterschiedliche Ideen. Zum einen gibt es die Idee eines „vollwertigen“ Heimarztes, der im günstigsten Fall alle Bewohner in einem Heim betreut, und, ähnlich wie im Krankenhaus, regelmäßige Visiten abhält $(C A 07 / 56)$. Der Vergleich zur Krankenhaustätigkeit bzw. -organisation wird recht häufig genannt. Vorteile eines Heimarztes werden darin gesehen, dass es einen fixen Ansprechpartner gibt und somit mehr Kontinuität in der Versorgung herrscht. Dieser Arzt sollte dann, „wie im Krankenhaus" im Heim vor Ort sein (BA06/106). Zum anderen gibt es die Vorstellung, einen Konsiliardienst aus einem oder mehreren Hausärzten einzurichten, der Visiten im Heim abhält (CA10/96). 
Auch die Vorstellung, dass sich mehrere Ärzte zusammenschließen könnten und dann abwechselnd Dienste im Heim ableisten, existiert (CA10/92), ebenso wie die Möglichkeit eines Stationsarztes (CA07/56). Als Alternative zu einem im Heim stationierten Arzt wird außerdem die Idee von einem "mobilen Hausarzt" genannt. Dieser wäre rein für die Versorgung von Pflegeheimbewohnern zuständig, könnte aber am Tag mehrere Pflegeheime visitieren (BA08/76).

"Integrierte Versorgung" ist das, was sich andere Hausärzte als Zukunftsoption vorstellen und wünschen. Die Idee, Krankenhauseinweisungen dadurch zu minimieren, dass der Hausarzt mit einem Raum im Heim vor Ort ist, in dem häufige Interventionen wie beispielsweise die intravenöse Flüssigkeitsgabe bei exsikkierten Patienten vom Hausarzt durchgeführt werden, wurde jedoch laut Aussage der Ärzte von Seiten der zuständigen Kassenärztlichen Vereinigung abgelehnt (AA01/153-161). Für Andere würde ein solches Konzept bedeuten, dass der Hausarzt als Heimarzt mit seiner Praxis vor Ort wäre. Dadurch ließe sich auch die Anzahl der Krankenhauseinweisungen reduzieren, da diese Art der Versorgung „fast den Status eines Krankenhauses annehme“ (BA08/70).

„....und ich denke, dass es wahrscheinlich die bessere Lösung wäre [...] dass es extra Ärzte gibt die Pflegeheime betreuen." (BA05/169)

\section{Vorteile eines Heimarztkonzepts für den Hausarzt}

Bei einer Versorgung durch einen Arzt können sich „alle aufeinander einstellen“. Interviewte Hausärzte sprechen damit die Verlässlichkeit und Vertrautheit zwischen Arzt, Pflegeheimpersonal und Bewohner an.

„,.. gab es ja mal [...] diese Heimärzte für die großen Heime. Also jemand, der auch wirklich vor Ort war, der alle Patienten versorgt hat und ich glaube, das hat eine Menge Vorteile. [...] und ich glaube, so ein Heimarzt hat schon was Gutes. Weil sich dann alle auch aufeinander einstellen können." (AA08/148)

Ärzte, die als Heimärzte tätig sind, ergänzen, dass es einen persönlichen und professionellen Profit von der Tätigkeit als Heimarzt geben kann. Der enge Pa- 
tientenkontakt und die Fokussierung auf geriatrische Patienten können die klinischen Fähigkeiten dieser Ärzte verbessern. Der zeitlich und räumlich engere Kontakt zu den Patienten lässt es darüber hinaus zu, Verläufe und Therapien besser zu beobachten und deren Erfolg einzuschätzen.

„Die Arbeit ist noch mal ganz anders als im Krankenhaus und ist in manchem sehr viel tiefgehender und man hat sehr viel mehr Begegnungen mit Menschen und das finde ich sehr interessant und man sieht auch Verläufe von Krankheiten, die man anders gar nicht beurteilen könnte. Man kann also die Wertigkeit von Therapien viel besser einschätzen und ich glaube, dass ich meine eigenen therapeutischen Fähigkeiten dadurch sehr viel besser schulen konnte [...]." (CA10/34)

\section{Problematiken eines Heimarztkonzepts}

Eine Problematik, die bei allen unterschiedlichen Vorstellungen zur Umsetzung des Heimarztkonzeptes erwähnt wird, ist die freie Arztwahl. Hier sehen die interviewten Hausärzte mögliches Konfliktpotenzial. Einem Patienten, der nach seinem Heimeinzug von seinem Hausarzt weiterbehandelt werden möchte, einen vorhanden Heimarzt „Vorzuschreiben“, wäre hinsichtlich des ethisch hohen Gutes der freien Arztwahl für viele Hausärzte eine problematische Situation. Das langjährige Vertrauensverhältnis zwischen Patient und Hausarzt wird hier als besonders schützenswert erwähnt. Gleichwohl wird bemerkt, dass auch bei Krankenhausaufenthalten ein neues Arzt-Patienten-Verhältnis entsteht und dies nicht grundsätzlich mit einem Mangel an Vertrauen einhergeht (CA09/76-77) (BA08/70).

Hausärzte, die einer Versorgung durch Heimärzte kritisch gegenüber stehen, bezweifeln unter anderem, dass dies die medizinische Versorgung verbessern würde. Die Arbeit als Heimarzt wird als zu einseitig empfunden, und es werden Einbußen in der medizinischen Qualität gesehen. Aus Sicht von manchen Ärzten hat eine tägliche Visite durch einen Heimarzt oder einen Arzt mit einer täglichen Sprechstunde im Heim auch für die Bewohner keinen zusätzlichen Nutzen. Es wird erwähnt, dass die Beschwerden und damit auch die Interventi- 
onsmöglichkeiten meist gleich bleiben und nicht in einer häufigeren Frequenz einer Anpassung bedürfen.

Ein Heimarztkonzept scheint dennoch für eine Vielzahl der Hausärzte ein mögliches Zukunftskonzept in der Versorgung von Heimpatienten zu sein.

\subsubsection{Allgemeine Lösungsvorschläge hinsichtlich der Arbeitssituation}

Hausärzte entwickelten in den Interviews nicht nur Lösungsvorschläge bzw. wünsche zur Zukunft ihrer strukturellen Berufsausübung wie dem Heimarztkonzept. Auch allgemeine Lösungsvorschläge zu den Arbeitsbedingungen und abläufen im Heim wurden erwähnt.

Ein wünschenswertes Ziel aus Hausarztsicht ist die Schaffung einer Kommunikationskultur im Sinne eines „runden Tisches“. An diesem Qualitätszirkel könnten Vertreter aus Politik, Heimleitung, MDK, Pflegekräften und Hausärzten beteiligt sein. Regelmäßige Treffen könnten den Austausch und die Verbesserung der Kommunikation unter den Beteiligten fördern und neue Leitlinien zur Versorgung von Heimpatienten könnten gemeinsam erstellt werden. Von allen Beteiligten gemeinsam erarbeitet, würden diese die Versorgungsqualität von Heimbewohnern verbessern und gleichzeitig für beispielsweise die Hausärzte ein freieres und qualitätsorientierteres Arbeiten möglich machen (CA09/61). Um die Arbeit im Pflegeheim für die Zukunft zu erleichtern und zu verbessern, würden Hausärzte die Zeit für solche Sitzungen investieren (CA09/73). Dabei steht für einen Teil der Hausärzte der Wunsch nach besserer Kooperation aller Beteiligten im Vordergrund. Teambesprechung mit allen (heimintern) an der medizinischen Versorgung Beteiligten erleben einzelne Hausärzte als äußerst bereichernd. Besonders, da ein persönlicher Kontakt untereinander entsteht (AA02/198). „Man muss von allen Seiten aufeinander zugehen“, scheint ein wichtiger Aspekt zur Verbesserung der Zusammenarbeit zu sein (CA09/74-75). Qualitätszirkel unter den Hausärzten, die ein Heim betreuen, gibt es vereinzelt schon. Von solchen regelmäßigen Treffen wird von einigen interviewten Ärzten berichtet (BA06/64). 
Die Kommunikation mit den Pflegekräften zu verbessern, steht für einen anderen Teil der Ärzte im Vordergrund. Hierbei wird mehr Potenzial gesehen, als in einer Erhöhung der Besuchsfrequenz. Dies empfänden diese Hausärzte als eine Verschwendung von Ressourcen, da es lediglich ein Ansatz an der Quantität, nicht aber zwangsläufig an der Qualität wäre (BA04/164). Als ökonomischer Lösungsvorschlag wird angebracht, dass Heime selbst die Budgetverantwortlichkeit für Bereiche wie Wundmaterial übernehmen sollten. Dadurch würde das hausärztliche Budget entlastet werden, und es würde zu weniger Konfliktpotenzial zwischen Heim und Arzt bei Verordnungen kommen (AA07/253). 


\subsubsection{Wahrnehmungen und Bedürfnisse der Hausärzte hinsichtlich der Versorgung und Betreuung der Bewohner}

Frage zwei kann durch die Ergebnisse der Auswertung in drei Teilaspekte unterteilt werden. Diese drei Aspekte sind erstens die Bewohnersicherheit in der medizinischen Versorgung, zweitens die Aufgaben der Pflegekräfte aus Hausarztsicht sowie drittens allgemeine Vorstellungen und Wünsche zur Bewohnerversorgung. Die interviewten Hausärzte konnten viele Punkte nennen, die sie sich für die Heimbewohner wünschen würden. Die Sicherheit und Kompetenz in der Versorgung der Pflegeheimbewohner durch die Pflegekräfte ist für die Hausärzte ein wichtiges Thema, mit einem hohen emotionalen Anteil. Hieraus resultierend wurden von den Hausärzten Bedürfnisse und Vorstellungen zur Bewohnerversorgung entwickelt.

Da die Versorgung der Bewohner auch das emotionale Erleben und die Wahrnehmung des Arbeitsumfeldes der Hausärzte beeinflusst, wird dies bei Frage drei nochmals Erwähnung finden.

\subsubsection{Bewohnersicherheit in der medizinischen Versorgung}

Umsetzung von ärztlichen Anordnungen und Sicherheit in der Medikamentenausgabe

Hausärzte geben an, dass die Umsetzung von ärztlichen Anordnungen oft mangelhaft sei. So wird von nicht umgesetzten Medikamentenanordnungen berichtet, die, besonders bei akuten Erkrankungen, die gesundheitliche Sicherheit des Bewohners gefährden. Viele Hausärzte sehen es als eine "gefährliche Pflege" an, wenn Medikamente nicht nach Anordnung oder unüberlegt gegeben und zum Teil auch vertauscht werden (AA05/41). Als ein Beispiel wird berichtet, dass ein Bewohner erneut in ein Krankenhaus eingeliefert werden musste, nachdem die medikamentösen Anordnungen nach Krankenhausentlassung von den Pflegekräften nicht übernommen wurden (AA01/89). Physiotherapieanordnungen wurden nach der Erfahrung von anderen Ärzten nicht weitergegeben und durchgeführt. Dies wird als „Zumutung für die Bewohner“ empfunden 
(BA10/33). Als ursächliche Fehlerquelle sehen Teile der interviewten Ärzte eine ungenügende Kommunikation in der Übergabe einzelner Schichten bzw. einzelner Pflegenden an (CA03/26) (CA04/22).

"Ich denke es kommt, wird ganz häufig so sein, dass bestimmte Dinge nicht umgesetzt werden die angeordnet werden." (CAO3/26) „...und dann auch letztendlich echt eine gefährliche Pflege zum Teil gemacht haben. Also die Antibiotika weiter gegeben haben, ohne Sinn und Verstand und da Medikamente vertauscht haben und das war schon immer irgendwie schwierig." (AA05/41)

Andere Hausärzte dagegen berichten von einer zuverlässigen Umsetzung der Anordnungen.

„Also das klappt da speziell in dem Heim eigentlich gut, eigentlich in allen Heimen wo ich bin klappt das gut, dass man da auch sich drauf verlassen kann, dass das was man anordnet auch umgesetzt wird."(CA06/73)

Pflegekräfte können die Patientensicherheit erhöhen, indem sie aufmerksam Medikamentenpläne überblicken und hinterfragen. So können bei Antibiotikaverordnungen bekannte Allergien eines Patienten von den Pflegekräften erinnert und der Arzt darauf aufmerksam gemacht werden.

„Die positiven Beispiele [...] also es hat sicherlich auch schon mal den Fall gegeben, dass ich zum Beispiel einem Patienten ein bestimmtes Antibiotikum verordnet habe und zwei Stunden später [...] kam der Anruf der zuständigen Stationsschwester die mir dann kurz mal mitteilte, na das Antibiotikum hat doch der Patient vor einem Jahr schon mal gehabt und damals hat er es nicht vertragen. Also das heißt die Rückmeldung, [...] dass sozusagen der Patient damals ich glaube sogar allergisch darauf reagiert hatte. [...] und das wäre natürlich auch wieder eine positive Rückkopplung für mich sozusagen hinsichtlich der Patientenbehandlung, wenn es aufmerksames Pflegepersonal gibt." (CA 03/32) 
Ein anderer Aspekt ist, dass es für Hausärzte wichtig erscheint, dass die Medikamente von den Pflegekräften gerichtet werden. In Phasen, in denen dies die zuständige Apotheke übernommen hatte, wurde ein größeres Fehlerpotenzial wahrgenommen (BA07/59).

Bezüglich der Bewohnersicherheit in der medizinischen Versorgung durch Pflegekräfte sehen Hausärzte oftmals Verbesserungspotenzial. Es wird jedoch auch der Wunsch deutlich, Pflegekräfte und Hausärzte mögen gemeinsam, und gewissermaßen unter gegenseitiger Kontrolle, zum Wohle des Patienten agieren. Besonders was pflegerische Aufgaben anbelangt werden konkrete Aufgabenbereiche und Handlungsweisen genannt, die Pflegekräfte, aus ärztlicher Sicht, für den Bewohner erfüllen und leisten sollen. Im Vordergrund steht der Wunsch der Hausärzte, ihre Patienten optimal versorgt zu wissen. Die Aufgaben der Pflegekräfte betreffend, äußern die von uns interviewten Hausärzte konkrete Vorstellungen und Wünsche:

\subsubsection{Aufgaben der Pflegekräfte aus Hausarztsicht}

\begin{tabular}{|l|}
\hline Zeit für Gesundheitsförderung \\
\hline Sichergestellte Grundversorgung und -pflege \\
\hline Termine bei Fachärzten \\
\hline Regelmäßige Kontaktmöglichkeiten für Angehörige \\
\hline Wahrung der Persönlichkeitsgrenzen der Bewohner \\
\hline Umfassendes Beschäftigungsangebot \\
\hline Individualität \\
\hline
\end{tabular}

Abbildung 7: Übersicht zu den Aufgaben der Pflegekräfte aus Hausarztsicht

\section{Zeit für Gesundheitsförderung}

Für ihre Patienten wünschen sich Hausärzte, dass sich die Pflegekräfte mehr Zeit für die Bewohner nehmen würden bzw. könnten. Hierin sehen Hausärzte 
auch die Chance, gesundheitlichen Problemen vorzubeugen bzw. diese zu verbessern. Als Beispiele werden Vorbeugung und Milderung von Osteoporose durch Mobilisation von Bewohnern nach draußen und dadurch Förderung der Vitamin-D-Bildung (CA08/40), Mobilisation zur Vermeidung von Dekubiti und Prophylaxe von Depressionen durch Verhinderung von Vereinsamung genannt. Hausärzte erleben es als frustrierend, wenn diese Maßnahmen von den Pflegekräften nicht geleistet werden können. Es soll Erwähnung finden, dass wohl auch die meisten Pflegekräfte sich die Zeit für mehr Gesundheitsförderung wünschen würden.

„Ich habe heute Morgen überlegt, was ist so die schlimmste oder schlechteste Erfahrung für mich im Altenheim. Das ist sicherlich zu sehen, wenn jemand nicht gut versorgt ist, wenn jemand ein Dekubitus entwickelt oder depressiv wird, weil er eben gar nicht mehr rauskommt und auch im Pflegeheim vereinsamt. [...] das ist die schwierigste Situation, wenn man dann sagt „da müsste sich was ändern" und man hört „dafür haben wir keine Zeit, das können wir nicht leisten." Das ist frustran für mich wenn kein Weg zu finden ist." (CA08/108)

\section{Sichergestellte Grundversorgung und -pflege}

Auch der Wunsch nach funktionierender Grundversorgung und -pflege wird von Hausärzten geäußert.

„Also klar ich würde mir halt wünschen, dass die Pflegekräfte natürlich erst mal die Grundversorgung sichern [...] das muss einfach funktionieren in dem Heim, dass die Patienten einfach versorgt sind $[\ldots]$ ". (CA07/42)

\section{Termine bei Fachärzten}

Ein spezieller Punkt kommt bei einzelnen Hausärzten zum Tragen: Die Pflegekräfte im Heim werden in der Verantwortung gesehen, die Terminorganisation bei anderen Fachärzten für die Bewohner zu übernehmen. 
„Die wissen, dass es ihre Aufgabe ist oder man sagt, der Patient muss zum Augenarzt [...] Termin ausmachen, Transport genehmigen lassen, ist deren Aufgabe." (BA09/22)

Dem widersprechen andere Aussagen, in denen solche Aufgaben klar als zum Zuständigkeitsbereich der hausärztlichen Praxis gehörend definiert werden.

„Das sozusagen macht dann mein Praxisteam und wir haben dem Heim signalisiert, ganz klar gesagt, um was wir uns kümmern werden und um was die sich kümmern sollen. Wir kümmern uns um Termine bei anderen Ärzten, bestellen den Transport, organisieren, dass der Patient abgeholt und wieder dorthin gebracht wird." (AA01/13)

Klare Organisationsstrukturen und Absprachen zu den Zuständigkeitsbereichen scheinen demnach eine wichtige Voraussetzung zu sein.

\section{Regelmäßige Kontaktmöglichkeiten für Angehörige}

In die Lage eines Angehörigen eines Pflegeheimbewohners versetzt, äußern andere Hausärzte den Wunsch, nach einer allumfassenden, sowohl physisch als auch psychisch guten Versorgung des Bewohners und den Wunsch nach regelmäßigen Kontaktmöglichkeiten mit Pflegekräften und Ärzten.

„Als Angehörige würde ich mir wünschen, dass meine zu pflegenden Angehörigen dort körperlich wie seelisch gut versorgt sind und ich regelmäßig erfahre wie es ihnen geht. Also das regelmäßige Gespräche möglich sind mit dem Pflegepersonal und mit dem betreuenden Arzt." (CA08/60)

\section{Wahrung der Persönlichkeitsgrenzen der Bewohner}

Es wird von einer oft wahrgenommenen Infantilisierung der Bewohner berichtet und von einem Nicht-Einhalten der Persönlichkeitsgrenzen. Die Hausärzte äuBern die Erwartung, dass die Pflegekräfte diesbezüglich besser geschult werden müssten und die einzelne Pflegekraft in ihrer Persönlichkeitsstruktur stär- 
ker auf einen respektvollen Umgang mit den Bewohnern, unter Berücksichtigung derer Gegenwart und Vergangenheit, zu achten habe.

\begin{abstract}
„[...] das ist einmal respektvoller Umgang mit Hilfsbedürftigen, weil da finde ich halt gelegentlich auch die Art und Weise wie die Patienten angesprochen und wie mit ihnen umgegangen wird, dass auf so einer Kind-Ebene gesprochen wird oder auch so diese Einhaltung der Persönlichkeitsgrenzen, dass das oftmals irgendwie verloren geht finde ich." (BA04/128)
\end{abstract}

\title{
Umfassendes Beschäftigungsangebot
}

Es wird sich für ein ausgewogenes aktives Angebot für die Bewohner ausgesprochen. Je mehr ein Pflegeheim auf ein umfassendes Beschäftigungsangebot achtet und je mehr aktive Ansprache auch seitens der Pflegekräfte erfolgt, desto weniger benötigen Bewohner den Hausarzt zur Kompensation von Langeweile und Tristesse.

„Wenn das ein Heim ist wo die Patienten einfach so stoisch vor sich hingesetzt werden, da gibt es eben Frühstück Mittagessen Abendessen, dann kommt vielleicht mal einer vorbei der irgendwie eine Singstunde macht aber ansonsten sind die den großen Teil des Tages auf sich alleine gestellt, dann ist so die Arztvisite zwei Mal im Quartal das Highlight. [...] Je mehr in einem Heim geboten wird, desto zufriedener sind die Patienten und desto weniger brauchen sie den Doktor als Highlight." (BA03/124)

Der Wunsch nach mehr Beschäftigungsangebot für die Bewohner taucht in den meisten ärztlichen Interviews auf. Dieser Wunsch basiert in vielen Fällen auf der wahrgenommenen fehlenden persönlichen Betreuung der Bewohner.

„...das geht nicht darum hier Krankengymnastik zu machen, sondern sich mit der Frau zu beschäftigen. Ja und das wird halt nicht getan, da wird halt viel Verwaltung gemacht und es fehlt halt einfach an der Betreuung der Patienten. [...] das persönliche Angebot, ja das fehlt halt." (BA01/127) 
Dem widersprechen Aussagen von anderen Ärzten. Die Bewohner im Pflegeheim werden hierbei als gut aufgehoben wahrgenommen und Hausärzte erleben in vielen Heimen echte Zuwendung von Seiten der Pflegekräfte.

„Dann es gibt Heime, wo sagen wir mal die Zuwendung für die Bewohner auch sehr positiv ist, sehr schön ist, auch wo die Bewohner eben nicht nur gepflegt werden, sondern wo sie eben auch Zuwendung bekommen." (BA03/42)

Die Ansätze und Überlegungen, wie mehr Beschäftigungsangebote konkret aussehen könnten, sind dabei sehr vielfältig. Ein konkreter Lösungsvorschlag entspringt dem Problem eines bei älteren Menschen oftmals aufgehobenen Tag-Nacht-Rhythmus bzw. dem Problem der (Durchschlaf-) Störungen bei älteren Menschen. Als Ansatz wird hierfür die Implementierung eines nächtlichen (Gesprächs-) Angebots genannt. Teilweise wird dies in Pflegeheimen als „Mitternachts-Café" schon umgesetzt.

Viele Ansätze beschäftigen sich mit dem Thema, ein „aktives Altern“ zu ermöglichen und zu gestalten. Hausärzte scheinen nach Heimeinzug ihrer Patienten oft einen raschen geistigen Abbau ihrer Patienten zu beobachten. Durch vermehrte Beschäftigungsangebote, die besonders auf eine Stärkung der Bewohnerautonomie und den Erhalt von Selbstständigkeit und die Übernahme von Verantwortung für einzelne Bereiche abzielen sollten, könnte dies, laut den befragten Hausärzten, verhindert werden. Die Aufgabe der Pflegekräfte und der Heimleitung sehen viele Hausärzte dabei darin, die Bewohner zu Aktivitäten zu animieren, Bewegung zu fördern und Angebote wie beispielsweise eine Beteiligung an der Essenszubereitung zu bieten. Die geistige Aktivität und Sozialkontakte zwischen den Bewohnern zu fördern, sehen Hausärzte darüber hinaus als Teil einer „aktivierenden Pflege“ und somit als Aufgabe des Pflegepersonals.

„,...und dann bauen die so schnell geistig ab, also für mich müsste es irgendwie noch so eine Mittelstufe geben, irgendwie so eine WG wo die noch Essen schnippeln und mehr Eigenverantwortung übernehmen. Aufgaben noch haben, ja also selber noch irgendwie in irgendeiner Weise kreativ sich einbringen müssen um ihr Alltäg- 
liches zu erleben [...]" (CA05/141)

,...nicht nur die Pflege auch die Anregungen an Patienten was sie machen können. [...] Die sollten auch drauf achten, dass die Patienten zum Beispiel bei gutem Wetter raus können, dass sie genug Sonnenlicht kriegen, dass Bewegung da ist. Das kann nicht alles über Physiotherapie laufen, das muss in Heimen auch auf anderen Wegen gewährleistet werden, dass Sozialkontakte da sind ist was, was das Heim organisieren muss." (CA08/38)

Um die körperliche Aktivität zu fördern, erzählen einige Hausärzte von positiven Erfahrungen mit Trommelkursen für die Bewohner oder gemeinsamer Gartenarbeit der Bewohner (CA06/103).

$\underline{\text { Individualität }}$

Auch die Betreuung der besonderen Patientenklientel der Demenz-Erkrankten beschäftigt die Hausärzte bei ihren Vorstellungen zur Bewohnerversorgung.

Demenz-Patienten durch individuelle Ansprache und durch Einbeziehungen der individuellen Lebensgeschichte ein Gefühl von Vertrautheit geben, wird von Hausärzten erwünscht und bei Umsetzung durch das Pflegepersonal als sehr positiv geschätzt (CA06/97) (CA06/49). Hierfür kann schon eine, von zum Beispiel Familienmitgliedern vormals verwendete, individuelle Ansprache hilfreich sein. Die Wahrung und Förderung von Individualität nennen viele der befragten Hausärzte als wichtige Aspekte im Umgang mit Heimbewohnern. Die Persönlichkeitsstruktur eines Bewohners zu erfassen und Wünsche und Bedürfnisse der Bewohner zu erkennen und umzusetzen, sehen die Hausärzte dabei als wichtigen Bestandteil der pflegerischen Aufgaben. Gleichwohl erkennen die Hausärzte an, dass dies unter bestehendem Zeit- und Personalmangel nur schwer umzusetzen ist.

„...um einmal rauszukriegen, was die Leute auch wirklich im Innersten wollen und das dann auch umzusetzen ja. "(CA06/89) 
„Was sie können, sollen sie auch tun“. Diese Aussage bringt es auf den Punkt, welches Maß an Selbstständigkeit für die Bewohner in einem Pflegeheim wünschenswert sein sollte (CAO9).

Den Bewohner als Ganzes sehen, als vollständigen Menschen wahrzunehmen, auch mit seiner Krankheit, das wünschen sich Hausärzte von den Pflegekräften. Hierin wird auch eine mögliche Stärkung der interprofessionellen Zusammenarbeit von Arzt und Pflegekraft gesehen. Einer interessierten und engagierten Pflegekraft, die an ihrem zu versorgenden Bewohner in all seinen Aspekten, eben auch in seiner Krankheit, interessiert ist, erklären Hausärzte gerne medizinische Zusammenhänge.

„...das wünschte ich mir manchmal auch, dass die Pfleger einfach nicht nur das Pflegerische sehen sondern einfach den Menschen als Ganzes, eben auch als Ganzes mit seinen Krankheiten. Da beantworte ich auch gerne Fragen wenn ich merke, da kommt ein Feedback, die nehmen da was mit von." (BA03/118)

Bezüglich der Aufgaben der Pflegekräfte aus Hausarztsicht sind also das Erkennen und Mitteilen von medizinischen Problemen der Bewohner, mehr Zeit für die Förderung von Gesundheitszuständen und eine kompetente und ausreichende Grundpflege wichtige Kernpunkte und Wünsche der Hausärzte an die Pflegekräfte. Darüber hinaus beschäftigen sich viele der gewünschten Aufgaben mit dem Ausbau individueller und fördernder Beschäftigungsangebote. Das Heim soll aus Hausarztsicht ein aktives Altern ermöglichen, in möglichst vielen Facetten. In vielen Pflegeheimen Deutschlands wurden diesbezüglich schon konkrete Ideen umgesetzt. 


\title{
5.2.2.3. Weitere allgemeine Vorstellungen und Wünsche zur Bewohner- versorgung
}

\author{
Mehrgenerationenversorgung \\ Höherer Personalschlüssel \\ Problem der passiven Aggressivität
}

Abbildung 8: Übersicht zu den allgemeinen Vorstellungen und Wünschen zur Bewohnerversorgung

Mehrgenerationenversorgung

Um dem vorzubeugen, dass geistig "fitte“ Bewohner im Heim die Ansprache und Erfüllung kognitiver Bedürfnisse verlieren, wird ein Versorgungskonzept im Sinne einer Mehrgenerationenversorgung vorgeschlagen. Die ideale Heimstruktur könnte dabei auch eine Verbindung von Kindergarten und Pflegeheim sein. Eine Zusammenführung von Kindern und Senioren könnte die Ballung von alten kranken Menschen im Heim und eine damit einhergehende Vereinsamung und Zentrierung auf Altern und Leiden verhindern. Die derzeitig vorherrschende Heimstruktur wird von einem Hausarzt mit dem drastischen Begriff der „Isolationshaft“ belegt.

„Also ich würde immer bevorzugen, anstatt ins Pflegeheim so eine Mischung. Mein Ideal ist Kindergarten und Pflegeheim gemischt oder so etwas aber wir tun einfach Unrecht. Es gibt Leute, die körperlich gebrechlich sind, die ins Pflegeheim kommen und dann mit Leuten zusammensitzen die geistig nicht mehr da sind, verwirrt sind, die haben keinen Ansprechpartner. Das ist Isolationshaft." (CA07/108-109)

\section{Höherer Personalschlüssel}

Viele Hausärzte sehen die Notwendigkeit von einer insgesamt höheren Personaldichte im Pflegeheim (CA06/105) (BA08/60). So könnten ihre Wünsche für die Bewohner umgesetzt werden. Positiv erinnern sich die Hausärzte an die früher bestehende Betreuung von Heimbewohnern durch Zivildienstleistende 
oder heutigen Teilnehmern eines Freiwilligen Sozialen Jahres. Es wird beschrieben, dass die Heimbewohner durch den Kontakt mit den jüngeren Leuten „aufgelebt" seien (BA01/131).

\section{Problem der passiven Aggressivität}

Von anderen Hausärzten wird von einem unangemessenen und verbesserungswürdigen Umgang der Pflegekräfte mit den Bewohnern berichtet. Die Pflegekräfte werden als oftmals unterschwellig aggressiv den Bewohnern gegenüber erlebt $(A A 07 / 14)$.

Zusammenfassend ist zu sagen, dass Hausärzte ihre Patienten im Pflegeheim gut versorgt wissen wollen. Das beinhaltet die medizinischen und pflegerischen Maßnahmen ebenso wie die präventiven Angebote und eine gute psychosoziale Betreuung. Der Ausbau von vielfältigen und individuellen Beschäftigungsangeboten war vielen Hausärzten in unseren Interviews ein besonderes Anliegen. 


\subsubsection{Wahrnehmungen und Bedürfnisse bezüglich des Tätigkeitsbereichs Pflegeheim. Wahrnehmung des Arbeitsumfelds Pflegeheim - Das zentrale Phänomen der Zerrissenheit}

Bei der Auswertung der Interviews zum Thema Wahrnehmung des Arbeitsumfeldes Pflegeheim zeigte sich „Zerrissenheit" als zentrales Phänomen. Der Begriff Zerrissenheit umfasst die ärztlichen Einstellungen und Perspektiven hinsichtlich des Settings Pflegeheim sowie das emotionale Erleben der Arbeitssituation von Ärzten im Heim. Zerrissenheit bezieht sich auf verschiedene Bereiche des ärztlichen Handelns und Denkens. So fühlen Ärzte Zerrissenheit zwischen den Polen, einerseits den eigenen Qualitätsansprüchen gerecht zu werden und gute Arbeit leisten zu wollen, und andererseits im Rahmen der bestehenden Gegebenheiten interagieren zu müssen und den bestehenden Anforderungen gerecht zu werden. Da diese beiden Pole teils konträre Anforderungen mit sich bringen, fühlen sich die Hausärzte „zerrissen“. Im Folgenden wird das zentrale Phänomen Zerrissenheit anhand des paradigmatischen Modells nach Strauss und Corbin (Strauss und Corbin 2010) beschrieben. 
Nach Strauss und Corbin führen ursächliche Bedingungen zum Auftreten eines Phänomens, während intervenierende Bedingungen auf die Strategien im Umgang mit dem Phänomen wirken. Die Ärzte verfolgen im Weiteren unterschiedliche Strategien, um mit der Zerrissenheit umzugehen. Diese münden wiederum in unterschiedlichen Konsequenzen. Eine Übersicht bietet die folgende Abbildung.

\begin{tabular}{|c|c|c|c|}
\hline $\begin{array}{l}\text { Ursächliche } \\
\text { Bedingungen }\end{array}$ & $\begin{array}{l}\text { Intervenierende } \\
\text { Bedingungen }\end{array}$ & Strategien & Konsequenzen \\
\hline $\begin{array}{l}\text { Wunsch, gute Arbeit } \\
\text { zu leisten } \\
\text { - Anfallenden Anfor- } \\
\text { derungen gerecht } \\
\text { werden wollen }\end{array}$ & $\begin{array}{l}\text { - Organisatorischer } \\
\text { Rahmen im Heim } \\
\text { - Gesamteindruck vom } \\
\text { Heim } \\
\text { - Organisation und } \\
\text { Qualifikation der } \\
\text { Pflegekräfte } \\
\text { - Zusammenarbeit mit } \\
\text { den Pflegekräften } \\
\text { - Persönliche Ebene } \\
\text { zu den Pflegekräften } \\
\text { - Zustand der Bewoh- } \\
\text { ner } \\
\text { - Ansprüche von Be- } \\
\text { wohnern \& Angehöri- } \\
\text { gen } \\
\text { - Vorerfahrungen der } \\
\text { Hausärzte }\end{array}$ & $\begin{array}{l}\text { - Positive Annahme } \\
\text { der Situation } \\
\text { - Gestaltung der } \\
\text { Zusammenarbeit } \\
\text { mit den Pflegekräf- } \\
\text { ten } \\
\text { - Reduzierung von } \\
\text { Zeit und Kontakt } \\
\text { - Engagement } \\
\text { - Annahme der } \\
\text { (schwierigen) Si- } \\
\text { tuation } \\
\text { Achten auf eigene } \\
\text { Bedürfnisse }\end{array}$ & 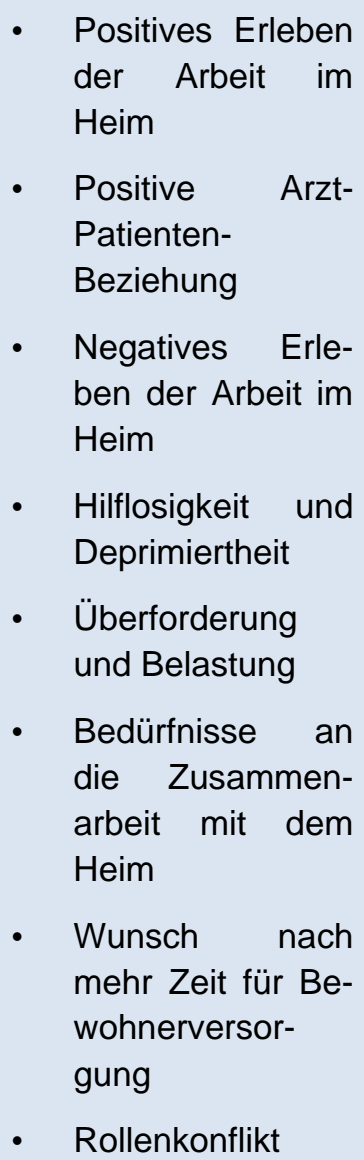 \\
\hline
\end{tabular}

Abbildung 9: Übersicht zu den ursächlichen und intervenierenden Bedingungen, Strategien, Konsequenzen 


\subsubsection{Ursächliche Bedingungen}

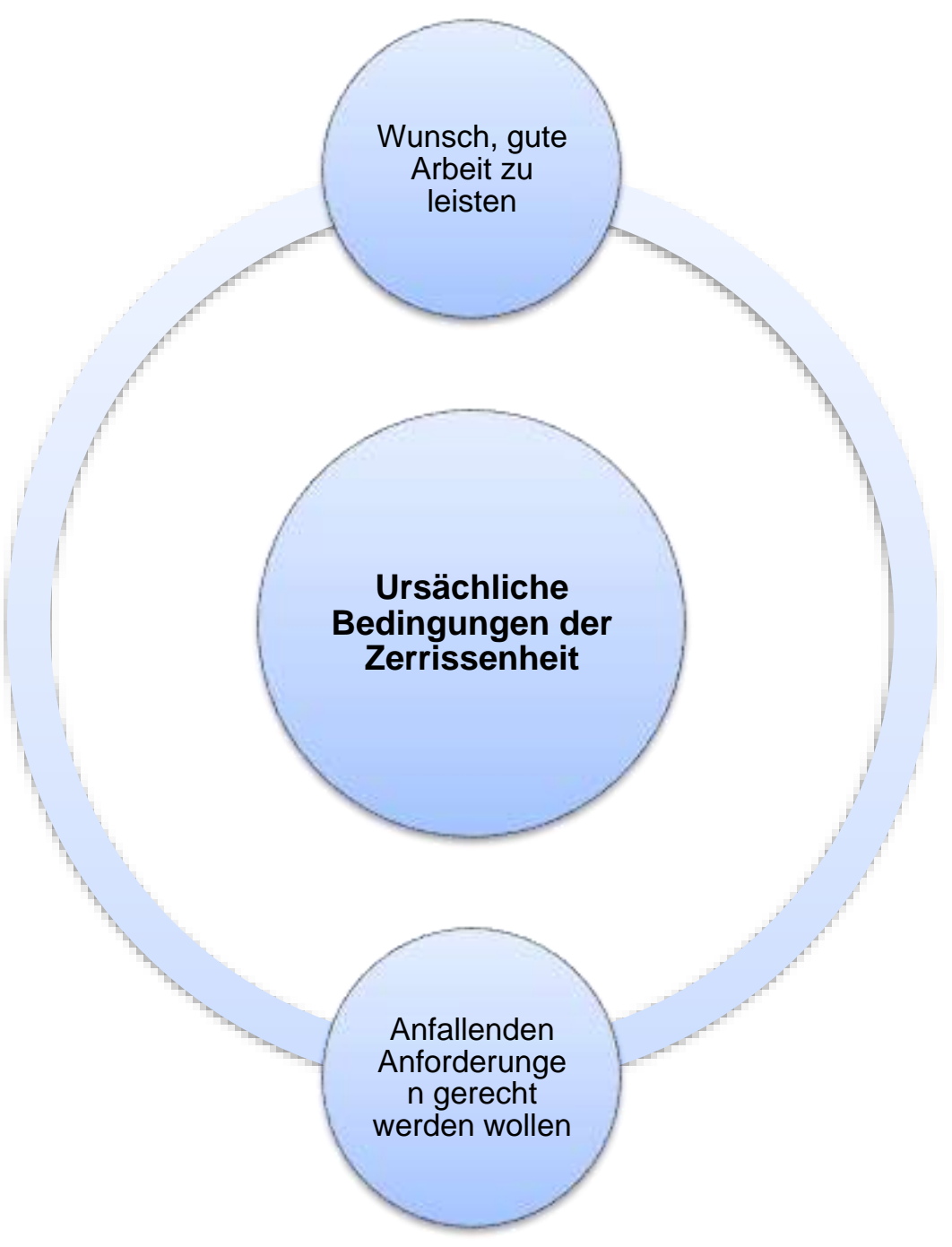

Abbildung 10: Ursächliche Bedingungen

Generell haben die Hausärzte den übergeordneten Wunsch, gute Arbeit zu leisten und den anfallenden Anforderungen gerecht zu werden. Diese Anforderungen beziehen sich auf die Bereiche eigene Praxis, Arbeit im Heim und Privatleben. Das resultierende Streben, möglichst vielen Anforderungen gleichermaßen gerecht zu werden, kann letztlich zum Gefühl der Zerrissenheit führen.

„....ich glaube immer wieder dieser Zeitfaktor, dass wir Ärzte ja na-

türlich in diesem Getriebensein, wir haben noch zwölf Hausbesu- 
che vor uns, und ich möchte eigentlich nicht abends um neun noch Hausbesuche im Altenheim machen." (CA03/48)

„Bist am Rand deiner Kapazität auch irgendwann angekommen, abends halb acht, haste irgendwann auch keine Lust mehr." (BA09/48)

\subsubsection{Intervenierende Bedingungen}

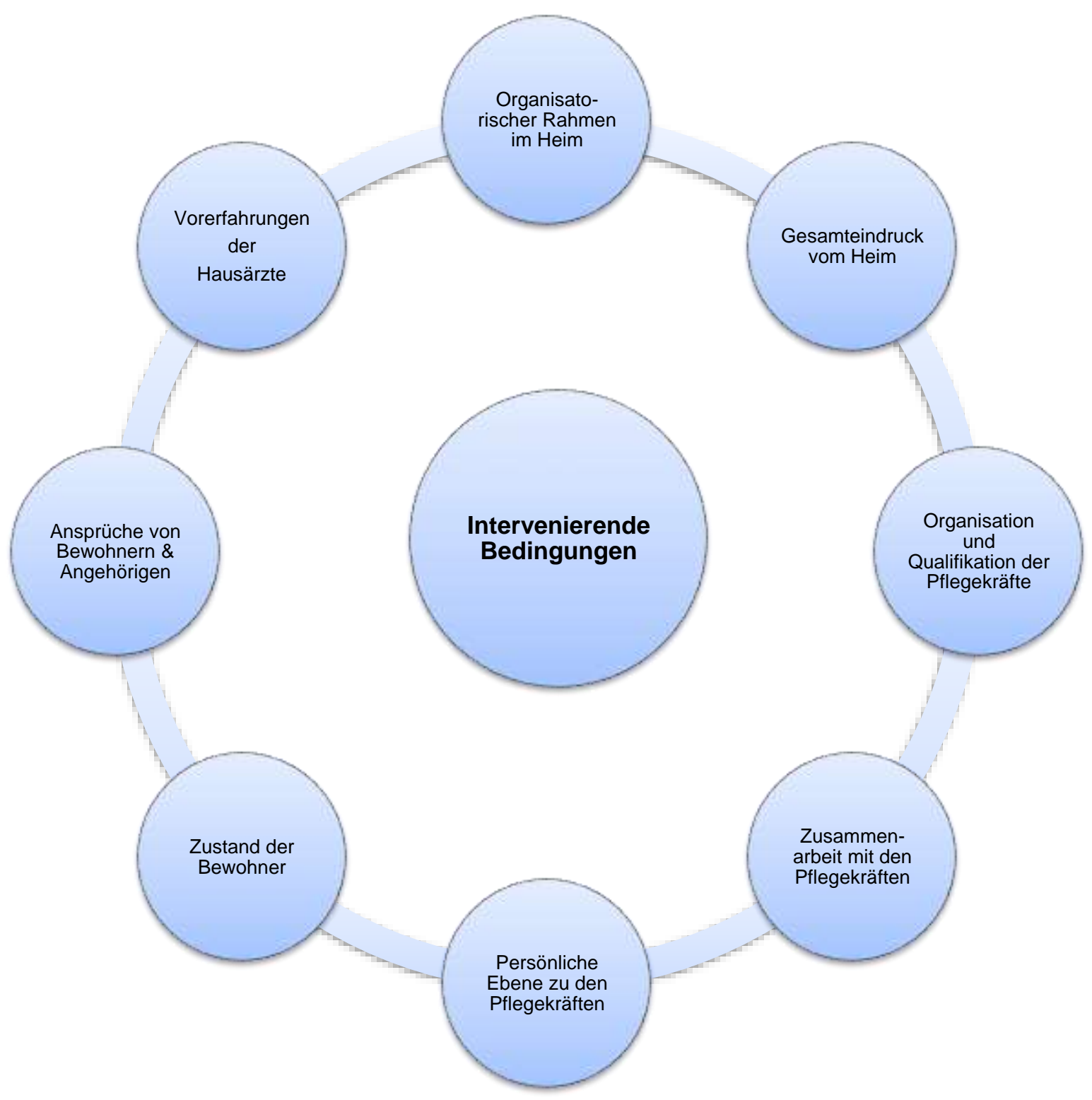

Abbildung 11: Intervenierende Bedingungen 
Die Strategien der Hausärzte mit Zerrissenheit hinsichtlich ihrer Arbeitssituation umzugehen, werden durch verschiedene Faktoren beeinflusst. Diese intervenierenden Bedingungen werden im Folgenden genauer dargestellt.

\section{Organisatorischer Rahmen im Heim}

Den organisatorischen Rahmen bilden Aspekte, die der Hausarzt im Heim vorfindet oder mit denen er bei seiner Arbeit im Heim konfrontiert wird.

Die Ärzte beschreiben, dass von Seiten der Heimleitung und der Pflegekräfte Druck auf sie ausgeübt wird, Dokumentationen zu Diagnostik und Therapie in den Bewohnerakten vorzunehmen oder auch per Fax wiederholt zu bestätigen (CA09/22). Dies stellt einerseits einen hohen zeitlichen Aufwand für die Ärzte dar und wird andererseits als Abgabe von Verantwortung und nicht-ärztlicher Aufgaben an die Hausärzte angesehen. Beide Faktoren tragen zu einer Verstärkung der Zerrissenheit bei und wirken sich auf die Strategien im Umgang mit der Zerrissenheit aus.

„...sodass wir Ärzte zu Tätigkeiten gezwungen werden sollen, die uns, die wir überhaupt nicht erfüllen müssen, beispielsweise eben die Ausführlichkeit der Dokumentation in den Pflegeunterlagen. Das ist nicht unsere Aufgabe, das ist Aufgabe der Pflegekräfte. [...] bedingt durch eine Überängstlichkeit, ein übermäßiger Kontrollzwang [...] der auch dazu führt, dass Ärzte wegen minderer Probleme zum Handeln naja gezwungen oder gebeten werden, wo das im Grunde genommen überhaupt nicht nötig ist. Wir würden aus unserer Sicht sagen, die Eigenverantwortlichkeit der examinierten Pflegekräfte hat immer weiter abgenommen, denen wird immer weniger Verantwortung zugebilligt und die Verantwortung wird uns aufgebürdet." (CA09/22)

„Damit sie nix falsch machen, wird der Arzt informiert. Der Arzt kann aber häufig am Telefon auch nur sagen, ,ja wenn ich das jetzt höre, kann ich doch daraus keinen endgültigen Schluss ziehen." Also muss er fahren, sich den Patienten angucken und so kommt eine Kette aufeinander, die rein aus Absicherung gemacht wird. 
Nicht, weil es dem Patienten notwendig ist Absicherung." (CA07/86)

Das Thema Verantwortung kommt in einem weiteren Punkt zur Geltung. Der Hausarzt trägt in der medizinischen Versorgung zwar die Verantwortung, muss sich bei der Umsetzung aber auf die Pflegekräfte teils blind verlassen und hat keine bis wenige Kontrollmöglichkeiten.

„...da muss ich drauf vertrauen, dass das gut läuft. Diese Kontrolle kann ich da nicht ausüben." (BA06/28)

Hausärzte sehen die letztliche Verantwortung bei sich selbst (CA01/26). Dieses Bewusstsein hat Einfluss auf die vom Hausarzt angewandten Strategien. Hinsichtlich der personellen Situation im Heim erleben Hausärzte die Arbeitssituation im Heim als positiver, wenn es ihnen möglich ist, kontinuierlich mit den selben Pflegekräften zusammenzuarbeiten. Besonders hervorgehoben werden konstante Ansprechpartner, denen ein hohes Maß an Kompetenz zugesprochen wird. Der häufige Wechsel von Personal wird als negativ und anstrengend empfunden. Im Umkehrschluss wirkt sich eine geringe personelle Fluktuationsrate positiv auf die Arbeitszufriedenheit der Hausärzte aus.

„...wo auch häufig Personalwechsel stattfinden, dass man jedes Mal, wenn man auf die Station kommt ein neues Gesicht sieht und sozusagen alles wieder von vorne anfängt. Da haben wir hier zum Glück ein Heim, wo es relativ kontinuierlich ist, das wir auch absichtlich betreuen, da macht es wirklich Spaß." (AA06/38)

Eine souveräne Haltung und Selbstständigkeit des Heims und eine gute Zusammenarbeit mit diesem wirken sich positiv auf die Strategien bezüglich der Zerrissenheit aus. Eine positive Annahme der Situation kann resultieren. Ein Effekt, der sich ergibt, ist, dass ein entsprechend souveränes und selbstbewusstes Auftreten von Heim und Pflegekräften auch die Souveränität und Autonomie der Bewohner stärkt.

„Positiv kann man sagen, dass also durchaus in einigen Heimen eine sehr gute Zusammenarbeit besteht, die auch mit einer meis- 
tens dann mit einer hohen Selbstständigkeit dieser Heime einhergeht. Das auch diese sehr souveräne Haltung des Heimes sich auf die Bewohner auswirkt und die also im hohen Maße auch autark weiterhin erscheinen..." (CA09/22)

\section{Gesamteindruck vom Heim}

Der allgemeine Eindruck, den ein Hausarzt von einem Heim hat, trägt ganz wesentlich zur Wahrnehmung des Arbeitsumfeldes bei und wirkt sich stark auf die Art und Weise aus, wie mit der Zerrissenheit bei der Heimtätigkeit umgegangen wird. Von vielen interviewten Hausärzten wird beschrieben, dass sie die Atmosphäre im Heim als unangenehm empfinden. Manche Hausärzte sind abgeschreckt von den hygienischen Verhältnissen im Heim.

allein die Atmosphäre mit der dicken Luft und der muffigen Luft [...] Macht es teilweise auch schwer das. " (AA03/33)

„Das und halt dann von der Hygiene her finde ich es halt auch teilweise, wenn ich da schon in ein Heim reinkomme und es stinkt nach Urin dann." (BA04/150)

\section{Organisation und Qualifikation der Pflegekräfte}

Es gibt intervenierende Bedingungen, die von Seiten der Pflegekräfte ausgehen und den Hausarzt in seinem Umgang mit der Zerrissenheit beeinflussen. So werden Sprachbarrieren durch mangelnde Deutschkenntnisse der Pflegekräfte sowohl für die Zusammenarbeit zwischen Hausarzt und Pflegekraft als auch für die Versorgung und Betreuung der Bewohner durch die Pflegekraft als äußerst negativ empfunden. Auch eine mangelnde Organisation der Pflegekräfte untereinander und Unterbesetzung der Pflegekräfte durch das Heim zählen zu den negativ beeinflussenden Faktoren. Werden die Pflegekräfte als unsicher in inrem Handeln und als nicht ausreichend qualifiziert wahrgenommen, verschärft dies den Konflikt im Bezug auf die Heimtätigkeit und das Phänomen der Zerrissenheit deutlich.

„Ja in anderen Heimen ist es einfach ganz schwierig. Da weiß der 
eine Arm nicht was der andere macht [...] und wie gesagt, was sehr schwierig ist für mich ist einfach, wenn zum Beispiel Kommunikation schlecht stattfinden kann weil die Altenpfleger nicht deutsch sprechen zum Beispiel oder einfach sehr sehr ängstlich sind, das ist auch schwierig für mich..." (BA03/46)

Hausärzte empfinden es als sehr ärgerlich, wenn Pflegekräfte ihre Kompetenzen überschätzen bzw. ihren Zuständigkeitsbereich überspannen. Als Beispiele werden eigenständige Medikamentengaben genannt.

„Also ich hab das mal erlebt, [...] da habe ich mich wirklich geärgert und da habe ich dann auch sicher was gesagt, ist, wenn die Pflege eigenmächtig etwas entschieden hat. So, also auch in Richtung Medikament [...] Kommt, ist wirklich ganz, ganz selten gewesen [...]. War auch nicht richtig. Ja, [...] auch wenn's richtig gewesen wäre, hätte ich Vetokarte gelegt, das geht nicht." (AA02/57-61)

\section{Zusammenarbeit mit den Pflegekräften}

Die Qualität der Zusammenarbeit mit den Pflegekräften im Heim beeinflusst die interviewten Hausärzte wesentlich bei der Wahrnehmung der Heime und spielt ebenfalls eine Rolle hinsichtlich der Ausprägung der Zerrissenheit. Einige Faktoren hinsichtlich der Zusammenarbeit fanden auch schon bei Frage zwei Erwähnung. Da die Zusammenarbeit mit den Pflegekräften auch das emotionale Erleben und die Wahrnehmung des Arbeitsumfeldes durch die Hausärzte stark beeinflusst, werden entsprechende Aspekte hier aufgeführt.

Hausärzte empfinden es als positiv, wenn sie sich die Verantwortung in der Bewohnerbetreuung mit den Pflegekräften teilen können. Auch, dass die Strukturen im Heim eine Teamarbeit und Visite wie im Krankenhaus zulassen, wird von den Interviewpartnern als günstig wahrgenommen. Die Ärzte fühlen sich positiv an ihre frühere Zeit im Krankenhaus erinnert und erleben als Folge dessen die Zusammenarbeit als angenehme Abwechslung zu ihrem autonom zu bestreitenden Praxisalltag. Dieses Besetzen der Heimtätigkeit mit früheren positiven Bedeutungsinhalten kann zu einer Reduzierung von Zerrissenheit führen. 
„Also mir gefällt das schon, muss ich sagen. Also die Situation mit einem Pflegeteam zusammenzuarbeiten. Das ist ja ein bisschen wie Krankenhaus. [...] das hat für mich so einen Krankenhausflair sozusagen, den ich auch gut finde." (AA10/100)

Hausärzte empfinden es hierbei als positiv, wenn ihre Wünsche von den Pflegekräften erfüllt werden.

„....man kommt an und kann speziell seine Wünsche äußern und in der Regel werden die auch erfült. Das ist halt das Positive in der Zusammenarbeit, dass das alles klappt." (CA04/28)

Wie bereits erwähnt, bemängeln viele Hausärzte bei den Pflegekräften ein fehlendes oder niedriges medizinisches Sachverständnis und Mitdenken in der Behandlung.

„...das ist schon frappierend, wenn dann Altenpfleger nicht wissen, was Heparin eigentlich ist. [...] oder wenn man morgens den Blutdruck misst, dass man sagt, man sieht „Oh, der ist ja ganz niedrig", dass man sagt: „Wir rufen erstmal an, bevor wir jetzt die anderen Blutdrucksenker auch noch geben." Ja? Anstatt wie eine Maschine zu sagen: „Die muss jetzt aber ihre Tabletten nehmen.“ (AA04/241)

Enttäuscht sind Hausärzte, wenn sie sich, entgegen ihrer Einschätzung, auf die Pflegekräfte nicht verlassen können und gesundheitliche Probleme der Bewohner verschleppt werden (AA08/104). Es wird darüber hinaus von Extremfällen berichtet, in denen Pflegekräfte nicht in der Lage waren, zu erkennen, dass ein Bewohner verstorben ist. Für Hausärzte schmälern solche Erlebnisse das Vertrauen in die Kompetenzen der Pflegekräfte und tragen zu einer verstärkten Zerrissenheit bei. Hausärzte können verschiedene Strategien entwickeln, um mit solchen Situationen umzugehen.

„....und letztendlich ist die Patientin dann tot [...] also die erkennen noch nicht mal, dass sie tot ist." (BA04/98) 
Gleichwohl wird diese Aussage dadurch revidiert, dass einige andere Hausärzte den Pflegekräften Kompetenz im Erkennen sterbender Patienten zusprechen und diese den Hausarzt und den Patienten möglicherweise von nicht mehr nötigen therapeutischen Verfahren abhalten.

„Wenn es zum Beispiel daran geht, dass Patienten womöglich sterben [...] denke ich manchmal, haben Schwestern oder Pflegekräfte einen besseren Blick, wann das tatsächlich so weit sein könnte und da kann es durchaus sein, dass ich also sozusagen noch irgendwelchen therapeutischen Impetus hab und die Schwester eigentlich schon erkannt hat, das ist ja eigentlich nicht mehr sinnvoll..." (CA10/79)

Hausärzte können die Zusammenarbeit mit den Pflegekräften als so schwierig empfinden, dass sie diese als Erschwernis ihrer ärztlichen Tätigkeit erleben. Begründet wird dies unter anderem mit fehlender Organisation, Motivation und Wissen auf Seiten der Pflegekräfte (BA10/15-17). Hieraus kann die Strategie der Reduzierung von Zeit und Kontakt folgen.

Durch einen Vertrauensmissbrauch von Seiten der Pflegekräfte kann die Zerrissenheit der Hausärzte verstärkt werden. Als Vertrauensmissbrauch wird empfunden, wenn die Pflegekräfte den Arzt bei Verordnungen übergehen.
„....also es kommt manchmal vor, dass Rezepte angefordert wer- den für teure Pflaster [...] und wir gar keine Diagnose haben [...] also mehrfach gefragt habe und nach zwei Wochen die Antwort kam: „Och, der hat gar nichts“. Also dann wurde das Pflaster of- fensichtlich für einen anderen Patienten gebraucht. Das ist ein Missbrauch meines Vertrauens." (CA08/84)

Hausärzte werden in ihrer Zerrissenheit weiterhin bestärkt und entwickeln dem Heim gegenüber ablehnende Strategien, wenn sie in die Versorgung nicht mit eingebunden werden und von den Pflegekräften übergangen werden. Dies kann Absprachen und Maßnahmen betreffen, die von den Pflegekräften mit den Angehörigen und unabhängig vom Hausarzt getroffen werden. Noch enttäuschender ist es für die Hausärzte, wenn sie von der Versorgung teils langjähri- 
ger Patienten abgezogen werden, weil die Pflegekräfte im Heim unzufrieden mit der Arbeitsweise des Arztes sind. Der Arzt hat hierbei kaum Rechtfertigungsmöglichkeit und ist dem Meinungsbild der Pflegekräfte teils ausgeliefert. Hausärzte fühlen sich hierbei „ausgebootet“ und empfinden solche Vorkommnisse als schlimm (AA10/102).

„Da hat das Pflegeteam mit den Angehörigen zusammen am Wochenende das Palliativteam gerufen und da fühlte ich mich nicht gut eingebunden..." (BA10/154)

Besonders die Hausärzte, die eine offene Kommunikation über problematische Vorkommnisse schätzen und praktizieren, sind frustriert, wenn ihre Kritik von Pflegekräften und der Heimleitung nicht aufgenommen und ernstgenommen wird.

„Wenn ich den Schwestern dann sage [...] „ich finde das nicht gut [...] sie haben mir gar nicht kommuniziert, dass der Patient da gestürzt ist“ [...] dann kriegt man halt mal Achselzucken entgegengebracht und ist halt so." (BA06/120)

Nicht nur die Zusammenarbeit, sondern auch die persönliche Beziehung zu den Pflegekräften beeinflusst den Umgang der Hausärzte mit und die Strategien in Hinblick auf die Zerrissenheit.

\section{Persönliche Ebene zu den Pflegekräften}

Es gibt Hausärzte, die von Misstrauen und Kontrollzwang von Seiten der Pflegekräfte berichten. Einen Grund hierfür sehen die Hausärzte in den strengen Vorgaben durch den MDK, denen die Pflegekräfte ausgesetzt sind.

„Weil immer dieser ominöse MDK ja kommen könnte und dann ist das nicht dokumentiert. [...] Und da fehlt mir so ein bisschen, finde ich, so das Vertrauen in eben meine hausärztliche Tätigkeit, dass ich auch die Verantwortung über das, was ich auch sage und nicht nur schreibe übernehme. Das immer so ein grundsätzliches Misstrauen ist. Das irgendwie so anerzogen wird. Alles das, was nicht 
schriftlich fixiert ist, ist nicht gesagt und nicht angeordnet. Und das ist was, was echt nervt." (AA05/45)

Auch gibt es Hausärzte, die sich von den Pflegekräften als Konkurrenz wahrgenommen und abgelehnt fühlen oder sich zum Teil von den Pflegekräften bei den Angehörigen von Bewohnern ausgespielt fühlen. Beides kann die Zusammenarbeit ungemein erschweren.

„Manchmal hat man auch so das Gefühl, als Arzt schlägt einem da erst mal so 'ne bisschen so eine Ablehnung entgegen." (BA05/94)

„Also das Negativste was ich so überlebt ha- erlebt hab, ist das einer den andern ausspielt. Also das die Pfleger gegen die Ärzte bei den Angehörigen der Patienten. Das finde ich, dass ist absolut negativ und kontraproduktiv. Weil das eine Menge Aufregung gibt und das gibt schon Heime, wo das schon mal eher vorkommt." (CA05/126-128)

Als positiver intervenierender Faktor wirkt es sich aus, wenn Pflegekräfte selbst Patienten beim Hausarzt sind. Dadurch entstehen eine besondere Vertrauensbasis und ein persönliches Verhältnis. Ebenso kann solch ein Verhältnis entstehen, wenn gegenseitiges Vertrauen über einen langen Zeitraum wachsen kann und eine vertrauensvolle Zusammenarbeit entsteht.

„Ich hab mit dem Personal keine Probleme. Die kommen auf mich $z u$, die sind zum Teil sogar inzwischen Patienten von mir und insofern gehen wir ziemlich persönlich miteinander um und dadurch kriegen sie natürlich auch Informationen, politischer Art, heimpolitischer Art, die sie vielleicht sonst, sich keiner trauen wird zu sagen." (BA07/155)

„....in einem anderen Heim [...] da habe ich mit Pflegekräften zu tun, die kenne ich schon seit zwanzig Jahren. Und das ist ganz toll. Die kennen ihre Leute, ich kenne die, ich weiß, wenn die mich anrufen, du musst kommen, dann komme ich." (AA01/191) 


\section{Zustand der Bewohner}

Die seelische und körperliche Verfassung der Bewohner spielt für die Hausärzte eine wichtige Rolle. Heimbewohner werden als schwerkranke Kohorte mit einem hohen Versorgungsbedarf gesehen. Die Betreuung dieser Patienten wird als sehr aufwendig beschrieben. Faktoren wie eingeschränkte Beweglichkeit und Mitarbeit bei Untersuchungen, Schwerhörigkeit oder Demenz unterscheiden die besondere geriatrische Klientel im Pflegeheim von jüngeren Patienten (AA02/22) (CA10/06).

„...dass in den Heimen ja sehr viel schwierigere Fälle sind als früher. Sie haben fast ausschließlich schwere Fälle, nicht? [...] Und das hat sich schon verändert." (AA07/317)

„Also das ist alles hier tatsächlich sehr viel aufwendiger und langwieriger oft." (CA10/06)

Diese Punkte sind unter anderem dann für die Ärzte so wichtig, wenn sie ihre eigene Arbeitszufriedenheit von der Zufriedenheit und der Versorgungssituation der Bewohner abhängig machen.

„Also ich messe es auch daran, wie zufrieden die Patienten sind. Und wenn die Patienten sagen: „Ach, Sie wissen ja, wie das hier läuft Frau Doktor und ich habe schon wieder stundenlang keinen Menschen gesehen" Also wenn die Patienten einfach auch zufrieden sind, das ist so - gut." (AA08/130)

Der Heimeinzug eines Patienten wird von Hausärzten oft durchaus befürwortet. Hausärzte erleben, dass es ihren Patienten im Heim besser gehen kann als zu Hause. Es ist eine schöne Erfahrung für Hausärzte und es reduziert ihre Zerrissenheit der Heimtätigkeit gegenüber, wenn sie sehen, dass ihre Patienten nach dem Heimeinzug aufblühen und körperlich sowie seelisch gesünder sind.

„Sehr positiv ist meine Erfahrung, dass viele Patienten die zuhause gerade noch klarkommen aber eben körperlich oder auch demenzbedingt zunehmend schlechter klarkommen, vereinsamen, 
depressiv werden, die dann ins Pflegeheim kommen und manchmal dort wieder gesunden, fitter werden, seelisch gesünder werden. Nicht mehr so depressiv sind und wieder aufblühen. Das ist eine sehr schöne Erfahrung." (CA08/40)

\section{Ansprüche von Bewohnern und Angehörigen}

Hohe Erwartungshaltungen und hohes Anspruchsverhalten der Bewohner wirken sich auf den Umgang der Hausärzte mit der Arbeit im Heim und damit auch auf deren Zerrissenheit aus. Hausärzte empfinden es so, dass Bewohner den Anspruch haben, der Hausarzt möge rund um die Uhr für sie bereit stehen.

„Also die Patienten die sich äußern können, legen in der Regel ein hohes Anspruchsverhalten an den Tag [...] Ärzte müssen immer Zeit haben, zu jeder Tages- und Nachtzeit möglichst zur Verfügung stehen und aber keine Wartezeiten und das ist immer ganz verblüffend. "(CA07/81)

Bewohner scheinen den Arzt als „Kümmerer“ für alle Lebenslagen anzusehen. Auch von Angehörigen werden teilweise überhöhte Anforderungen an den Hausarzt gestellt. Dies wird von den Hausärzten als Ausdruck einer Übertragung des eigenen schlechten Gewissens der Angehörigen und als Zeichen einer daraus resultierenden over-protection den Bewohnern gegenüber interpretiert.

„Die übliche Situation des schlechten Gewissens weil die Oma der Opa oder sonst jemand ins Heim abgeschoben sein soll und daraus eine Überfürsorge resultiert, die oftmals so weder vom Pflegepersonal noch von den Ärzten zu leisten ist, weil es einfach absurde Forderungen sind." (CA09/34)

\section{Vorerfahrungen der Hausärzte}

Manche Hausärzte haben selbst in der Pflege im Heim gearbeitet. Durch diese wichtige Vorerfahrung wird die Zerrissenheit reduziert und auch die Zusammenarbeit mit den Pflegekräften positiv beeinflusst. 
„Also ich muss vielleicht dazu sagen, dass ich selber anderthalb Jahre im Pflegeheim gearbeitet habe [...] und da sieht man das eh schon noch mal mit anderen Augen denke ich." (BA04/124)

Auch die unter Fragestellung eins und zwei beschriebenen Faktoren wie beispielsweise die ökonomischen Rahmenbedingungen können die Zerrissenheit mit beeinflussen. Sie wirken jedoch nicht so stark als einzelne intervenierende Bedingungen und sind deshalb hier nicht noch einmal gesondert erwähnt. 


\subsubsection{Strategien}

Strategien sind Handlungen, die ausgeführt werden, um mit dem Phänomen umzugehen, darauf zu reagieren, es zu erfüllen oder durchzuführen (nach Strauss und Corbin). Für das Phänomen der Zerrissenheit sind dies aktive Verhaltensweisen oder auch Einstellungen, die vom Hausarzt ausgehen. Sie werden von den zuvor genannten Bedingungen beeinflusst. Die Strategien zum Phänomen der Zerrissenheit lassen sich in mehrere Gruppen unterteilen:

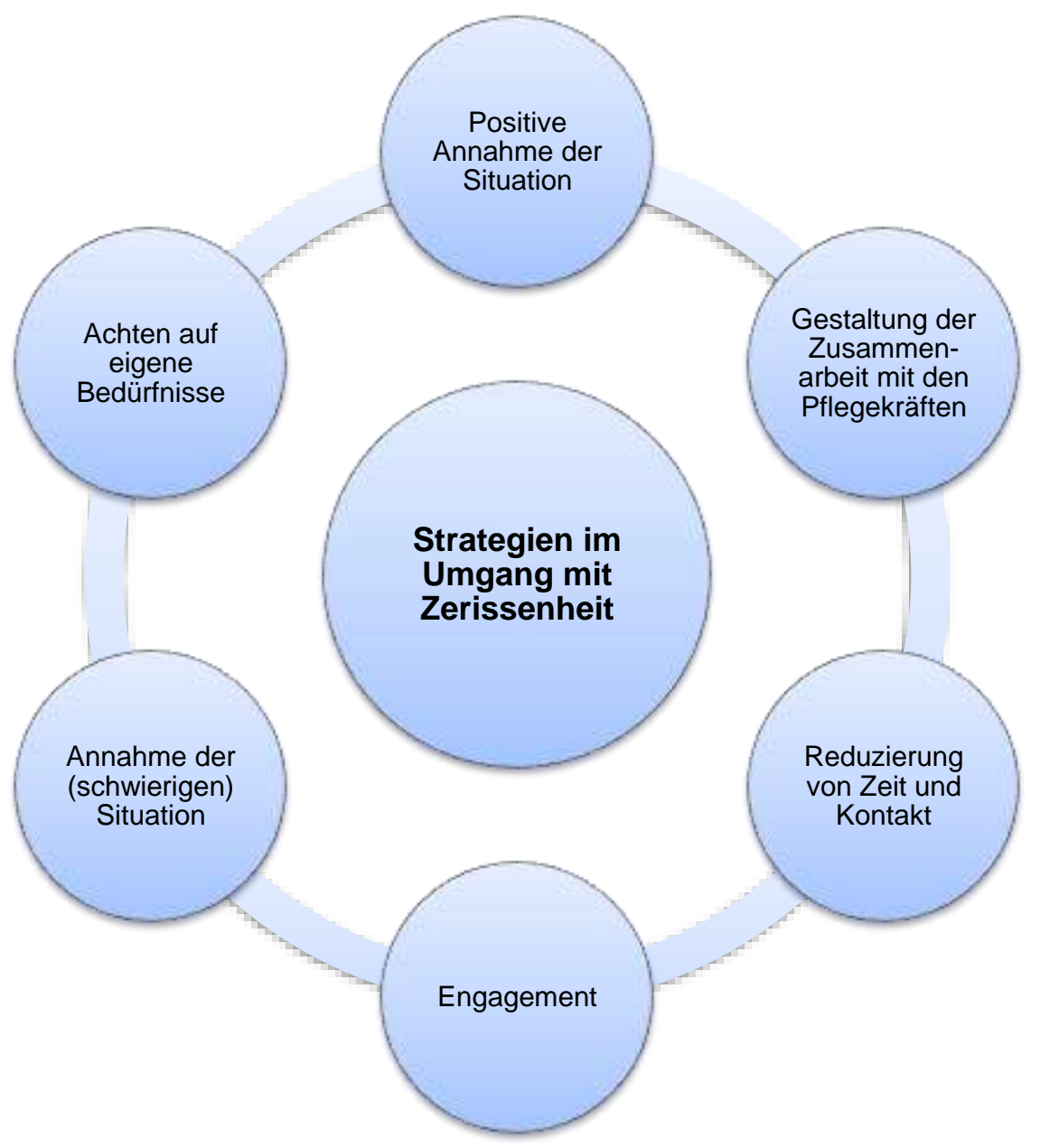

Abbildung 12: Strategien 


\section{Positive Annahme der Situation}

Unter anderem die intervenierende Bedingung, dass im Heim eine Teamarbeit und Visitentätigkeit wie im Krankenhaus möglich ist, wirkt auf die Strategie, dass Hausärzte die Arbeit im Heim als „nette Abwechslung“ zur Praxistätigkeit annehmen. Heim- und Praxistätigkeit gleichen sich dabei durch ihr unterschiedliches Patientenklientel und die unterschiedlichen Anforderungen aus. Für viele Hausärzte gehören beide Arbeitsbereiche zu ihrem Tätigkeitsbereich und werde als solche akzeptiert und auch geschätzt. Ein weiterer positiver Umgang mit der Zerrissenheit und somit eine Reduzierung eben dieser erfolgt durch die Freiheit, die sich Hausärzte in ihrer Arbeitsgestaltung nehmen. Die Arbeit im Heim bereitet den Hausärzten Freude und kann in dem Umfang und Rahmen ausgeübt werden, solange eben dies der Fall ist.

„Ja, das ist ja das wunderschöne an meinem Beruf: ich mache nur das, was mir Spaß macht!“ (AA01/205)

Schlussfolgernd daraus lässt sich erkennen, dass Hausärzte bei einem Mangel an Arbeitszufriedenheit und Freude an der Arbeit zur Strategie der Reduzierung von Zeit und Kontakt im Heim greifen. Diese wird in einem weiteren Abschnitt näher beschrieben.

\section{Gestaltung der Zusammenarbeit mit den Pflegekräften}

Hausärzte wenden verschiedene Strategien an, die zu einer Reduzierung der Zerrissenheit führen. Dazu zählen auch solche, die die Zusammenarbeit mit den Pflegekräften beeinflussen. Strategien zur Klärung möglicher Probleme in der Zusammenarbeit lassen Dimensionen erkennen: Von einigen Hausärzten wird die direkte und offene Aussprache mit den Pflegekräften gesucht und bevorzugt. Die Dimension reicht bis zu den Hausärzten, die sich mit Anregungen und Beschwerden zur Zusammenarbeit lieber direkt an die Pflegedienstleitung wenden und mögliche Probleme auf dieser Ebene klären. Dazwischen liegen die Strategien von Hausärzten, Teambesprechungen mit allen Ebenen einzuberufen, wenn Konflikte auftreten, oder sich erst bei fehlender Lösungsfindung mit den Pflegekräften an die Pflegedienstleitung zu wenden. 
„ICh frage immer ob alles in Ordnung ist und ich hoffe, dass sie ehrlich antworten." (CA08/96)

„Unfreundliches Personal gibt es. Da beschwere ich mich dann bei der Leitung, das geht relativ zügig und das wird dann auch verändert. Das habe ich bisher immer nur als konstruktiv erlebt." (AA02/37)

Einige Hausärzte sehen die Überwachung der Pflegekräfte, besonders im Bereich der Behandlungspflege, als ihre Aufgabe an und reduzieren dadurch ihre Zerrissenheit.

„...und in gewisser Hinsicht auch eine Überwachung der Pflege jetzt im Hinblick auf den allgemeinen Gesundheitszustand des Patienten." (CA09/32)

Andere zeigen aktiv Kooperationsbereitschaft, um die Zusammenarbeit zu verbessern. Beide Wege (Überwachung oder Kooperation) können die Zerrissenheit der Hausärzte auf ihre Weise reduzieren. Kooperation äußert sich darin, dass sich die Hausärzte offen für Vorschläge der Pflegekräfte zeigen, versuchen auf Augenhöhe und nicht hierarchisch zu kooperieren und generell die Pflegekräfte aktiv in die Behandlung der Bewohner mit einbeziehen.

„....und wenn man selber jetzt nicht so total ist, dass man den Arzt so raushängt und meint man wüsste alles immer am allerbesten, dann kann man auch gut miteinander zurechtkommen und kooperativ irgendwie miteinander zusammenarbeiten und wenn die dann noch nett sind, dann gehe ich da gerne hin." (BA05/94)

„....und ich versuch die Dinge irgendwie kooperativ zu besprechen und nicht hierarchisch." (BA05/98)

Kommt es zu Auseinandersetzungen zwischen Pflegekräften und Angehörigen, stellen sich Hausärzte oftmals schützend vor die Pflegekräfte. Dadurch verdeutlichen sie den Teamcharakter der Zusammenarbeit einerseits, übernehmen 
aber andererseits auch letztendlich die Verantwortung für die Behandlung der Bewohner.

„...dass so ein Missmut der Angehörigen gegenüber dem Pflegepersonal so aufkommt. [...] dann mische ich mich da ein und sage, dann sollen sie bitte mich anrufen. Es ist nicht so, dass ich mich immer vor die Schwestern stelle, aber doch oft." (AA05/35)

Des Weiteren sehen es Hausärzte als ihre Aufgabe an, den Pflegekräften medizinische Zusammenhänge in der Versorgung zu erläutern. Dies kann aus der intervenierenden Bedingung resultieren, dass Hausärzte einen Mangel an medizinischem Sachverstand bei den Pflegekräften kritisieren. Gemeinsame Fallkonferenzen oder Fortbildungen, die vom Hausarzt gehalten werden, stärken die Zusammenarbeit im Team und bringen für den Hausarzt den Vorteil, dass sein Vertrauen in die Kompetenzen und Fähigkeiten der Pflegekräfte gestärkt wird. So wird die Zerrissenheit reduziert. Darüber hinaus haben Fortbildungen den Effekt, dass die Pflegekräfte in medizinischen Belangen selbstsicherer und kompetenter werden und sich dadurch weniger oft beim Hausarzt rückversichern. Auch kann der Hausarzt durch Fortbildungen Einblicke in die ärztliche Sichtweise vermitteln und Einblicke in pflegerische Bedürfnisse erlangen (CA01/30). Dies fördert das gegenseitige Verständnis und wirkt sich positiv auf die Zusammenarbeit und damit auch auf die Zerrissenheit aus.

„Haben denen dann sozusagen erzählt, warum das so ist [...] Und dann merkte ich so bei zumindest so zwei, drei, dass so der Groschen fiel und die auch mal sich ein bisschen theoretisch darüber Gedanken machten, warum eigentlich oder was für eine Erkrankung die Patientin hat." (AA05/53)

„Die Fortbildungen gehen halt immer darum, dass man dem Pflegepersonal einfach Wissen an die Hand gibt. Weil ich immer denke, jedes Wissen hilft den Pflegekräften auch wiederum in ihrem Umgang sicherer zu werden und zu erkennen, wo ist eine Bagatelle, wo ist es ernst. " (CA07/92) 


\section{$\underline{\text { Reduzierung von Zeit und Kontakt }}$}

Wie bereits erwähnt können Hausärzte Strategien zur Reduzierung von Zeit und Kontakt hinsichtlich der Pflegeheimtätigkeit anwenden. Dies geschieht, wenn die Arbeitsbedingungen im Heim als entsprechend ungünstig empfunden werden. Betreffen können diese Strategien die Tätigkeit im Heim an sich, die Zusammenarbeit mit den Pflegekräften oder auch die Arzt-PatientenBeziehung. Sie dienen dazu, dem „Spannungsfeld Pflegeheim“ zu entgehen und damit die Zerrissenheit zu reduzieren. Die Zusammenarbeit mit den Pflegekräften und dem Heim betreffende Strategien diesbezüglich sind die Durchführung von Hausbesuchen als schnelle und strukturierte Visite ohne Beteiligung einer Pflegekraft (BA09/28). Wird die Zusammenarbeit als zu konfliktreich und ohne Anzeichen auf verbessernde Veränderungen erlebt, wird die Zusammenarbeit und der Kontakt zu den Pflegekräften gänzlich vermieden und eventuell die Betreuung von Patienten in den entsprechenden Heimen abgebrochen.

„... weil ich mich so oft geärgert habe und es gibt auch Pflegepersonal, wo ich denke: "Oh, da mache ich heute - minimal, minimal.“ Und auf einer Station lief es mal so schlecht, dass ich da auch gar nicht mehr ins Dienstzimmer gegangen bin. [...] - also das ist natürlich dann kein gutes ärztliches Verhalten, aber." (AA08/126)

„Ich habe mir angewöhnt mit Heimen zusammenzuarbeiten, wo ich denke, dass ist für mich in Ordnung die Zusammenarbeit. Wenn ich merke, dass klappt nicht, [...] dann sage ich auch: „Möchte ich nicht weiter machen." Die Freiheit nehme ich mir raus. “ (AA02/37)

Manche Hausärzte selektieren die Heime, die sie besuchen. So gehen viele Hausärzte bewusst und lieber in Heime mit guter Atmosphäre und Sauberkeit und dorthin, wo ihre Patienten auch pflegerisch gut versorgt werden und meiden Pflegeheime, in denen dies nicht gegeben ist.

„Also Pflegeheime habe ich jetzt im Notdienst auch schon gesehen, die waren so was von unter aller Kanone, dass man wirklich da nicht als Arzt mit drin stecken möchte [...]. Und das würde ich auch nicht machen. Da hätte ich auch keine Lust zu. Also da sol- 
che Patienten zu betreuen, die so schlecht gepflegt werden." (AA04/129)

Andere Hausärzte bieten darüber hinaus auch keine zeitintensiven Teambesprechungen an und distanzieren sich von diesen Wünschen und Anforderungen.

„Die "besondere Dementenabteilung", hatte früher auch immer regelmäßige Stationsbesprechungen [...] Ich wurde teilweise gebeten, daran teilzunehmen aber das war mir dann irgendwie zu viel Arbeit. Und die Zeit konnte, wollte ich dafür jetzt sozusagen nicht erübrigen." (AA03/48)

Auch der Kontakt zu Bewohnern kann eingeschränkt oder das Behandlungsverhältnis sogar abgebrochen werden, wenn keine gute Beziehung mehr zustande kommt. Stellen Bewohner für die Hausärzte Ansprüche, die ihren Aufgabenbereich überschreiten, so werden diese von den Hausärzten zurück gewiesen.

„Was ich meide, vermeide, was üblich war früher, ist sich auf das Sofa zu setzen und Händchen zu halten und leere Zeit zu füllen. Das mache ich nicht gerne. Ich tröste gerne, ich begleite gerne, aber was ich nicht gerne mache ist Langeweile füllen. Das mache ich nicht gerne. [...] Weil ich fühle mich ausgenutzt. Möchte ich nicht." (AA02/12)

„ICh kann kein Seelsorger sein [...] ich kann das in Akutsituationen aber ich kann das nicht als Dauerbetreuung machen." (BA04/178)

Auch eine fehlende Vertrauensbasis zwischen Arzt und Patient oder ein schlechtes persönliches Verhältnis können zum Abbruch der Behandlung durch den Hausarzt führen. Zu solchen Situationen kommt es jedoch nur in Ausnahmefällen. Hausärzte zeigen dabei, zum Beispiel durch das kommunizierte „Genervt-sein" von Patienten, ein wenig professionell anmutendes Verhalten. Die persönliche Ebene solcher konfliktreicher Beziehungen steht deutlich im Vordergrund. 
„...es gibt so ein paar Patienten, die wirklich marottisch sind oder einfach depressiv sind und die nerven total, weil die immer wieder sich nur um sich selbst drehen und das ist mir völlig uninteressant. [...] Also, das kann ich auch nicht lösen, ja? Und diese Zeit, die würde ich gerne nicht investieren. Habe ich auch jetzt, vor zwei, drei Monaten eine Patientin abgelehnt. [...] Und zwar hat die mich belogen, [...] das kam noch als I-Tüpfelchen dazu. Und da habe ich gesagt: „Wissen Sie was? die Vertrauensbasis ist einfach nicht mehr da. Ich würde es gerne beenden." "Ich beende es." Nicht „ich würde gerne", sondern: „Ich beende es." (AA02/123)

\section{Engagement}

Viele Hausärzte zeigen in der Zusammenarbeit mit den Pflegekräften und in der Bewohnerversorgung oftmals auch großes Engagement und bemühen sich um jeden ihrer Patienten. Sie haben ein Interesse an einem guten, vertrauensvollen Verhältnis mit den Pflegekräften und zeigen dies durch private Gespräche.

„... weil ich mich da auch um jeden persönlich bemühe." (BA10/73) „... und wir haben uns noch ein bisschen privat unterhalten mit der Pflege, wofür sonst so gut wie keine Zeit ist. Das fand ich aber sehr nett." (AA02)

Hausärzte übernehmen die Verantwortung für ihr Handeln und machen sich damit zu einem verlässlichen Partner in der Zusammenarbeit mit den Pflegekräften (AA05/45). Im Heim können sich Hausärzte intensiv um ihre Patienten kümmern, ohne sich verausgaben zu müssen. Dies gelingt durch die Betreuung der Bewohner im Team mit den Pflegekräften. Für Hausärzte ist dies eine wichtige Strategie, um die Zerrissenheit im Bezug auf die Tätigkeit im Pflegeheim

nicht wachsen zu lassen und sich selbst vor Konsequenzen wie Burn-out zu schützen.

„.... weil man da sozusagen schon auch dann die Strukturen so hat, dass man sich drum küm-, dafür sorgen kann, dass bestimmte Sachen gemacht werden." (AA04/105) 
„Vieles ist viel einfacher. Sie können organisieren, dass da jeden Tag eine Tablette genommen wird, ja? [...] Sie haben jemanden der alleine zuhause ist und regelmäßig stürzt, dann sind sie sehr ängstlich, dass das, was sie tun den Patienten mehr schadet, als nützt. [...] Und das ist im Heim einfach besser [...]" (AA04/113121)

Hausärzte übernehmen als "Case-Manager" die Funktion, die medizinische Versorgung des Bewohners zu koordinieren und zu überblicken. Dabei haben sie sowohl die Arbeit der Pflegekräfte als auch die von anderen Fachärzten geleistete Versorgung im Blick. Hausärzte überprüfen und überwachen insbesondere die Interaktion von Pharmaka, die von verschiedenen Fachärzten verordnet werden.

„Also ich bin der Meinung, dass der Hausarzt die medikamentöse Therapie zumindest überwachen sollte [...] und ich achte da schon darauf, dass das was der Neurologe oder wer auch immer verschreibt [...], dass das zumindest an Interaktion auch mit dem Rest passt. [...] Ich denke das ist vielleicht doch eher Sache des Hausarztes, der irgendwie die ganzen Disziplinen irgendwie zusammenbringen sollte." (BA06/76)

Einige Hausärzte sind bereit, sich sehr umfassend für das Heim erreichbar zu machen und als Ansprechpartner zur Verfügung zu stehen. Sie kommen damit dem Absicherungsbedürfnis der Pflegekräfte und dem Versorgungsbedarf und -wunsch der Bewohner nach. Hausärzte werden damit auch ihren eigenen hohen Anforderungen und dem bei einigen Ärzten bestehenden Rollenbild eines "guten Arztes", der ständig für seine Patienten ansprechbar ist, gerecht. Dies kann die Arbeitszufriedenheit fördern und damit die Zerrissenheit abbauen.

Die Strategie des Engagements bezüglich der Erreichbarkeit zeigt verschiedene Dimensionen. Einige Hausärzte machen sich in besonderen Situationen, sprich bei Palliativpatienten oder bei Sterbebegleitungen, für die Beteiligten erreichbar (AA03/33). Es gibt aber auch Hausärzte, die grundsätzlich fast rund um die Uhr ansprechbar und erreichbar sind. Ein außergewöhnliches Angebot stellt die Erreichbarkeit am Wochenende und im Urlaub dar. 
,...die Heime haben meine Privatnummer und wenn das so abzusehen ist, dass in bestimmten krisenhaften Verläufen [...] oder Sterbeentwicklung, dann biete ich denen auch an, dass die mich persönlich halt anrufen können." (AA03/33)

„... und über Mittag wenn wir den Anrufbeantworter haben, liegt das Handy griffbereit nebendran, sodass sich die Teams jederzeit wirklich sofort hier Gehör verschaffen können." (BA10/172)

„Aber auch da werde ich auf Handy angerufen. Also im Urlaub rufen die mich auch an." (AA04/87)

Das Durchführen von regelmäßigen Visiten ist Teil der hausärztlichen Tätigkeit im Heim und stellt bei entsprechender Frequenz ein außergewöhnliches Versorgungsangebot aus Sicht des Hausarztes dar. Die Präsenz im Heim fördert die Zusammenarbeit mit den Pflegekräften, trägt zu einer guten medizinischen Versorgung der Bewohner bei und reduziert somit die Zerrissenheit im Bezug auf die Heimtätigkeit (AA03/37).

In den meisten Fällen übernimmt der bisherige Hausarzt auch nach Heimeinzug seiner Patienten deren weitere Betreuung. Damit erzeugen Hausärzte eine Kontinuität in der Versorgung von Patienten. Sie fühlen sich ihren Patienten gegenüber verpflichtet, scheinen diese nicht „im Stich lassen“ zu wollen und sehen die Weiterbetreuung nach Heimeinzug auch als Teil ihres hausärztlichen Selbstverständnisses an.
„....auch wenn wir ein bisschen weiter fahren müssen und wenn es irgendwie ein bisschen blöd ist aber wir bemühen uns schon. Also hatte ich jetzt letztens jemand der ist 96 und geht mit 96 ins Heim. Da kann ich jetzt nicht sagen, der muss einen neuen Hausarzt ha- ben, dass finde ich wirklich schrecklich und das machen wir halt da schon." (CA06/45)

Kommt es doch zum Hausarztwechsel nach Heimeinzug, sind die bisherigen Hausärzte für den Wechselwunsch ihres Patienten in der Regel offen. 
„Ja ich bin da sehr offen, ich meine wenn die Patienten sagen sie möchten dann zu einem anderen Arzt, habe ich Verständnis dafür, habe ich auch kein Problem damit." (BA08/18)

Auch wenn, wie im Vorfeld erwähnt, einige Hausärzte einen seelsorgerischen Anteil ihrer Tätigkeit bewusst reduzieren oder ablehnen, so gibt es auch viele Hausärzte, die sich bewusst nicht nur für das medizinische, sondern auch für das seelische Befinden der Bewohner interessieren. Sie zeigen Engagement, indem sie sich bewusst zum Gesprächspartner für die oftmals sozial isolierten Heimbewohner machen. Die Kommunikationsfähigkeit der Bewohner zu erhalten und den Fokus der Interaktion nicht nur auf die körperlichen Beschwerden zu lenken, ist hierbei ein wichtiges Motiv. Für das seelische Wohlbefinden der Bewohner ist diese Form der sozialen Interaktion mit dem Hausarzt sehr bedeutsam. Hier spiegelt sich auch der von vielen Hausärzten vertretene Aspekt der ganzheitlichen Patientenversorgung, die auch die psychosoziale Gesundheit stark berücksichtigt, wider.

„Und da ich offen für alles bin, nicht nur die medizinischen Fragen, sondern auch die natürlich seelischen Fragen." (AA01/11)

„...und wie gesagt, dass begleitende Gespräch und da versuch ich irgendwas ein bisschen mal anzusprechen, wo die auch was zu erzählen vielleicht mal. Die sind ja doch eben zum Teil eben sozial isoliert. [...], insofern bin ich dann eben auch mal ein Gesprächspartner [...], was manchmal nicht unbedingt nur den medizinischen Bereich betrifft." (CA02/28)

„Und im Prinzip erzählen wir viel von allen möglichen Sachen, so von früher und es ist auch halt wichtig, dass man eine längere Zeit kommuniziert. [...] also bei diesen routinemäßigen Besuchen ist eigentlich viel Erzählen dabei..." (CA06/04)

Hausärzte übernehmen dabei nicht nur die Verantwortung für das rein medizinische oder körperliche Wohlergehen ihrer Patienten. Auch die mit der Behandlung einhergehende Beeinflussung der Lebensqualität und Lebensfreude von 
Patienten unterliegt für viele Hausärzte dem ärztlichen Aufgaben- und Verantwortungsbereich.

„Ein guter Arzt ist im Prinzip [...] jemand, der sich sehr gut Gedanken macht, was er seinen Patienten antut [...] Was nützt es mir bei einem 90-Jährigen einen top eingestellten Zucker zu haben, wenn er dafür keine Lebensfreude mehr hat." (CA07/52)

Wie jedoch schon beschrieben, gibt es auch Hausärzte, die über die medizinische Versorgung hinaus keinen Handlungsspielraum für sich sehen und nicht als "Seelsorger" auftreten wollen. Hierbei scheinen auch wieder die in Fragestellung eins behandelten Faktoren wie Zeit und damit auch die Vergütung eine Rolle zu spielen.

„Aufgrund der kurzen Zeitvorgaben meinerseits, ist das nicht so besonders häufig, dass ich ausführliche Gespräche mit den Patienten führe. Es geht meistens eher um medikamentöse, fachliche Fragen." (AAO3/27)

\section{Annahme der (schwierigen) Situation}

Eine Strategie mit (schwierigen) Situationen umzugehen, liegt in der Annahme solcher Situationen. So wird die teilweise belastende und anstrengende Arbeit im Heim als Teil der hausärztlichen Tätigkeit angesehen und als solche akzeptiert. Dieser Umgang hilft Hausärzten, ihrer Zerrissenheit zu begegnen.

„....ich denk ist einfach ein Teil unsrer Tätigkeit. Auch die Leute bis zu ihrem Tod zu begleiten." (BA09/52)

Im Umgang mit den Bewohnern haben sich viele Hausärzte damit abgefunden, oftmals nicht viel tun zu können und vor allem an der oftmals sozialen Isolation im Heim nichts ändern zu können (CA02/28). Was die Angehörigen angeht, so haben Hausärzte gelernt, mit überhöhten Ansprüchen und teilweise sogar Androhungen professionell umzugehen und diese nicht persönlich an sich heran zu lassen.

„... weil ich kann ja die Situation der Leute nicht ändern. Ne, also, 
das deprimiert einen ja auch selber und wenn man, man will gerne helfen, aber wenn die Kinder die Damen nicht besuchen, dann besuchen die Kinder die Damen nicht. Das kann ich nun als Arzt nicht ändern. " (AA04/55-61)

"Oh es gibt Menschen, die kümmern sich ganz arg, die rufen täglich an, vorwurfsvoll [...]. Bis hin zu Drohungen, wenn das und jenes nicht gemacht wird, dann wird das der Bild-Zeitung mitgeteilt oder was auch immer, also overprotectiv. [...] Kennen wir, müssen wir damit umgehen, haben's gelernt damit umzugehen, geht dann meistens gut." (BA02/83)

\section{Achten auf eigene Bedürfnisse}

Auf ihre eigene Arbeitszufriedenheit und ihre Bedürfnisse zu achten, ist für Hausärzte eine weitere Strategie, die Zerrissenheit nicht zu groß werden zu lassen.

„...das man dann guckt: Bewährt sich das oder bewährt sich das nicht? Und verbessert das die Arbeitszufriedenheit?" (AA07/315) 


\subsubsection{Konsequenzen}

Es lassen sich folgende Konsequenzen erheben:

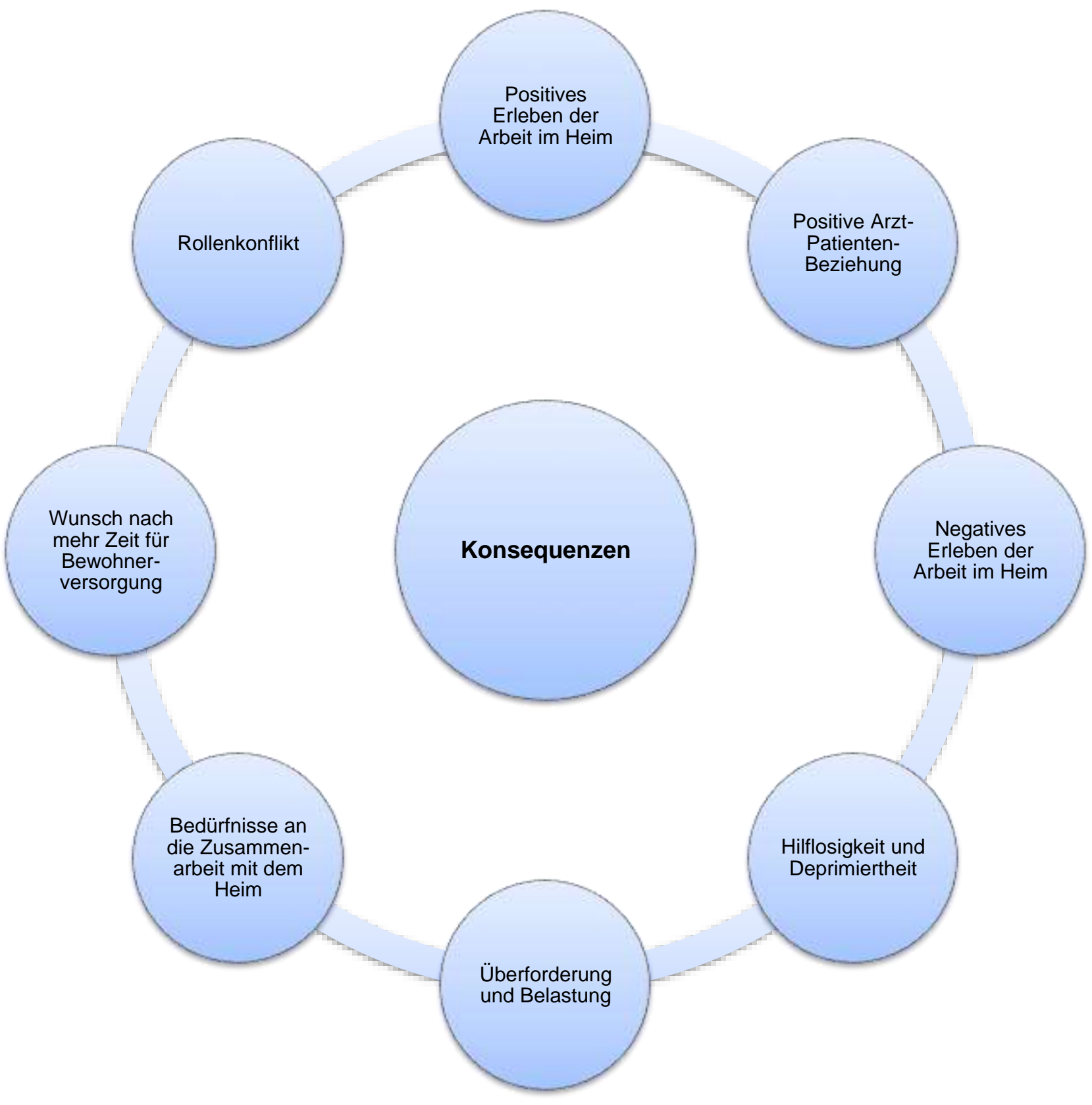

Abbildung 13: Konsequenzen 


\section{Positives Erleben der Arbeit im Heim}

Hausärzte erleben, wenn sie Strategien zur Reduzierung der Zerrissenheit anwenden, die Interaktion mit dem Pflegeheimpersonal und das Setting Pflegeheim als solches als angenehm und positiv. Wesentliche Bedingung für dieses positive Erleben, ist eine gute und wahre Zusammenarbeit mit den Pflegekräften.

„Das ist ein Pflegeheim oder eine Station im Pflegeheim wo ich sehr gern hingeh, weil ich mit den Schwestern da gut zurechtkomme und die mit mir auch." (BA05/58)

Viele Hausärzte empfinden Arbeitszufriedenheit bei ihrer Tätigkeit im Heim. Diese kann durch eine gefühlte moralische Höherstellung durch die Arbeit im Heim erreicht werden. Hausärzte haben einen ethischen Anspruch an sich, den Kranken und Alten der Gesellschaft beizustehen. Diesen Anspruch zu erfüllen, kann den Ärzten das Gefühl, moralisch gut zu sein, geben. Dies wird dadurch verstärkt, dass auch unter vielen ärztlichen Kollegen die geriatrische Arbeit eher abgelehnt wird. Viele Hausärzte scheinen dem Wertebild entsprechen zu wollen, wonach sich eine Gesellschaft daran misst, wie sie mit den Kranken, Alten und Schwachen umgeht. Aber auch der Aspekt des „Hausbesuche macht man halt“, also dass die Versorgung von Heimbewohnern Teil der hausärztlichen Tätigkeit ist, scheint sich hier wieder zu finden.

„Auf der einen Seite ist für mich also sehr befriedigend, ist diese Arbeit, weil ich weiß, dass viele Andere es nicht machen und auch so ein gewisser Anspruch, der ist, ja, woran soll sich die Gesellschaft messen, wenn nicht an den, an diesen Letzten, (die nicht für sich sorgen können)." (AA03/33)

\section{Positive Arzt-Patienten-Beziehung}

Der Hausarzt erlebt die Arbeit im Heim als Freude, wenn der Kontakt zu den Bewohnern positiv ist. Langjährige Beziehungen führen zu so einem vertrauensvollen Verhältnis. Durch die Kontinuität in der Betreuung können teilweise 
außerordentliche Beziehungen entstehen, die den Hausarzt auch persönlich bereichern und über ein rein professionelles Verhältnis hinausgehen.

„Manche Patienten kennt man schon sehr lange und da entwickelt sich natürlich auch - also auf der Beziehungsebene auch gute Verbindung und man freut sich, da hin zu gehen." (AA07/06)

„Also ganz besondere Beziehungen und die mir dann auch sehr gefehlt haben, als sie gestorben sind. [...] das sind aber mehr so Sternschnuppen." (AA10/80)

Des Weiteren erleben Hausärzte durch die Dankbarkeit und Wertschätzung ihrer Patienten eine große Bereicherung und Freude an ihrem Beruf. Die Dankbarkeit macht den Ärzten den Sinn ihrer Tätigkeit nochmals bewusst, und das Gefühl, „etwas bewegen zu können“, steigert die Arbeitszufriedenheit. Auch wird die Arbeit und der Kontakt mit älteren Patienten, besonders von eher jüngeren Hausärzten, als bereichernd erlebt.

„Also es ist schon so, dass man diese Wertschätzung genießt, die man da hat. Also das ist schon so, dass man das Gefühl hat, dass man da ordentlich was bewegen kann. [...] Also ich persönlich mag ältere Menschen gerne und unterhalte mich sehr gerne mit denen. Und finde das immer sehr bereichernd." (AA04/103)

\section{$\underline{\text { Negatives Erleben der Arbeit im Heim }}$}

Aus dem Phänomen der Zerrissenheit kann sich die Wahrnehmung der Hausärzte einer allgemein als nicht zufrieden stellende (Allgemein-) Situation im Heim ergeben.

„Ich bin mit der jetzigen Situation oftmals sehr unzufrieden. Wünsche mir deswegen sehr stark, dass sich an dieser Situation in $\mathrm{Zu}$ kunft einiges ändert." (CA09/73)

Eine kontraproduktive Zusammenarbeit mit den Pflegekräften führt auch zu einer negativen Wahrnehmung des Arbeitsumfeldes. Von einigen Hausärzten wird das Pflegeheim als ein Spannungsfeld empfunden. Heimleitung, Pflege- 
kräfte und Hausärzte arbeiten gegeneinander. Es entstehen Interessenskonflikte und Kommunikationsprobleme. Die gestörte und konfliktreiche Kommunikation zwischen den einzelnen Beteiligten führt zu Spannungen und Spannungen ziehen hierbei als Konsequenz auch ein angespannt Sein der Hausärzte nach sich. Die Wahrnehmung des Arbeitsumfeldes ist durch so eine Situation verständlicherweise nicht gut.

„...gewisses Spannungsfeld sich aufgetan hat zwischen Heim, Heimleitung, Pflegekräften und auf der anderen Seite den ärztlichen, den für die Patienten zur Verfügung stehenden Ärzten." (CA09/22)

„,...also das ist sehr konfliktträchtig [...] es entstehen an vielen Stellen Reibungspunkte, die sich aus sich selbst heraus ergeben. [...] die aber aus meiner Sicht eigentlich lösbar wären, wo aber wenig, also aus unserer Sicht jedenfalls wenig, von der anderen Seite da ist, da auf uns zuzugehen." (CA10/38)

\section{Hilflosigkeit und Deprimiertheit}

Für einige Hausärzte überwiegen allgemein im Bezug auf die Arbeit im Heim negative Erfahrungen.

„Ich fürchte, ich habe nur eine schlechte Geschichte. Es ist dann doch mehr Schlechtes als Gutes." (AA08/72)

Andere Hausärzte spezifizieren ihre Gefühle und Wahrnehmungen der Arbeit im Heim gegenüber genauer. Es wird beschrieben, dass die Arbeit im Heim depressiv und hilflos machen kann. In diesem Gefühl spiegeln Hausärzte die Situation und den Umgang mit den zum Teil einsamen und deprimierten Heimbewohnern wider.

„Das Thema Resignation, Depression ist natürlich bei dieser Klientel relativ hoch. [...] für mich erlebe ich das manchmal als ein bisschen zu viel zum Teil, also es ist nicht so, dass ich da immer glücklich von dannen ziehe, sondern dass ich da auch einiges mit- 
nehme an Deprimierung oder Depression. Das ist schon zum Teil bedrückend, also auch weil, weil ich kann ja die Situation der Leute nicht ändern. Das deprimiert einen ja auch." (AA04/55)

Hausärzte sind betroffen, wenn sie die Pflege ihrer Patienten als „technischen“ Ablauf wahrnehmen, die menschliche Zuwendung vermissen lässt. Zwar wird von den Hausärzten differenziert, dass auch eine gute Pflege im Heim familiäre Bindungen wohl nicht zu ersetzen vermag, die bestehenden Angebote scheinen in der Mehrzahl jedoch nicht ausreichend zu sein.

„...das alles sehr technisiert dann auch wirkt ist mein Eindruck, also ist jetzt nicht das Medizinische sondern das Menschliche was fehlt oder was nicht fehlt aber was erweitert werden kann und soll." (BA08/60)

„....was ich mir halt wünschen würde, dass es nicht häufig nur ne Aufbewahrungsanstalt ist. Ja, sondern das tatsächlich mit den dort ein-, den Insassen sozusagen ja auch entsprechend was getan wird und das ist also ausgesprochen wenig." (BA01/127)

Von einigen Hausärzten werden Pflegeheime als „Aufbewahrungsanstalten“ empfunden, in denen zwar für Nahrung und medizinische Versorgung gesorgt wird, allerding nicht hinreichend menschliche Zuwendung besteht oder sogar eher ein abgeklärter Umgang mit den Bewohnern herrscht. Auch die Wahrnehmung der Bewohner als fremdbestimmt und mit nicht hinreichendem Respekt behandelt, macht viele Hausärzte persönlich betroffen.

„Es hat mich schon sehr, menschlich betroffen sage ich mal, die Situation in den Heimen, jetzt aus menschlicher Sicht und das ist ja Aufbewahrungsanstalt, also die Menschen werden dort gut versorgt, haben was zu Essen, was zu Trinken, haben ein Dach über'm Kopf in Anführungszeichen. So pauschal das klingen mag aber das Menschliche, dieses Familiäre, das fehlt natürlich, ja." (BA08/92)

„... habe ich den Eindruck, dass durch den täglichen Kontakt mit 
den Bewohnern, viele von den Pflegekräften aus den Augen verlieren, dass die Menschen am Ende ihres Lebens stehen. Und das da so automatische Abläufe stattfinden, die ich persönlich für mich nicht gut heißen kann." (AA07/22)

„Das ist ja ein Albtraum im Altersheim zu sein, mit einer Fremdbestimmung von Leuten, die ein Viertel so alt sind wie man selber, einen Duzen zum schlimmsten Falle, ja?" (AA05/101)

Der eigene Heimeinzug wäre demnach für viele befragte Hausärzte ein „Albtraum". Die Hausärzte wünschen sich, selber einmal nicht im Pflegeheim versorgt werden zu müssen.

„Ich wünsch 'mir, nie im Pflegeheim zu landen.“(BA09/105)

„...ist für mich ein Albtraum.“ (CA07/105)

\section{Überforderung und Belastung}

Schaffen es Hausärzte durch die Anwendung von Strategien zum Umgang mit der Zerrissenheit nicht, diese zu reduzieren, resultiert oftmals ein Gefühl der Überforderung mit der Situation im Heim.

„...da fühle ich mich in meiner Funktion völlig überfordert und bin dem nicht zugewandt." (CA07/81)

Dies wird durch den empfundenen Zeitdruck und fehlende Pausen in der täglichen Arbeit weiter verstärkt und der Hausarzt zusätzlich belastet. Die Arbeit erweist sich dann nicht nur als psychisch, sondern auch als physisch belastend.

„....manchmal herrscht ein ganz schöner Zeitdruck. Eile, Bedrängnis. Ich komme also manchmal dann hierher, also nach der Sprechstunde und eine sehr schöne zeitdichte und intensive Tätigkeit. [...] Das sind dann schon, zehn, elf Stunden Tage ohne, ohne bedeutsame Pause. Und das ist dann einfach anstrengend, also, das ist dann manchmal schon... recht belastend." (AA03/35) 
Eine weitere Belastung kann durch eine schlechte Versorgungssituation der Bewohner entstehen. Eine mangelhafte pflegerische Versorgung im Heim macht die Ärzte persönlich betroffen und ist belastend.

„....und das dort ein Mangel an Versorgung ist, der einfach überall bekannt ist und einfach auch zu einer schlechten Versorgung der Leute führt. Ist eben manchmal auch eben nicht so gut auszuhalten. Das belastet mich halt schon." (AA03/35)

Hausärzte können die Arbeit im Heim als belastend und teilweise schwer auszuhaltend empfinden. Das Heim wird als Ort des Kummers und der Krankheit erlebt.

„...in dem Pflegeheim erlerne, lerne ich eine Ballung von Kummer kennen. Je kränker sie werden, desto mehr werden sie zentriert. Auch die Gespräche in Altenheimen drehen sich ganz wirklich nur noch um Krankheiten." (CA07/102-107)

\section{Bedürfnisse an die Zusammenarbeit mit dem Heim}

Für ihren als hoch empfundenen Aufwand durch die Arbeit im Heim erwarten viele Hausärzte optimale Bedingungen bei dieser. Zu diesen, vom Heim an Strukturen zu erfüllenden Bedingungen zählen die Reduzierung der Wartezeit für den Hausarzt, so dass er zügig und strukturiert seine Aufgaben erledigen kann und klare Informationen erhält.

„....wenn man sich als Arzt - und vor dem heutigen Hintergrund der Kondition - die Mühe macht ins Heim zu gehen, sollte es zeitnah Bedienung geben, keine lange Wartezeiten, klare Information und das wissen die auch." (AA02/37)

\section{Wunsch nach mehr Zeit für Bewohnerversorgung}

Hausärzte wünschen sich, mehr Zeit für die Versorgung von Heimbewohnern zur Verfügung zu haben und damit einen höheren Versorgungsstandard bieten zu können. 
„ICh selber hätte an mich den Anspruch, dass ich für Bewohner für besser hielte, ich könnte öfter ins Pflegeheim gehen." (BA10/160)

\section{$\underline{\text { Rollenkonflikt }}$}

Eine übergeordnete Konsequenz, die sich sowohl aus den Strategien zur Zerrissenheit als auch aus den Ergebnissen der ersten und zweiten Forschungsfrage ergibt, ist der Rollenkonflikt von im Heim tätigen Hausärzten. Damit spiegelt diese Konsequenz das zentrale Phänomen der Zerrissenheit besonders gut wider.

Es sind hierbei die verschiedenen Rollen zu berücksichtigen, die die Person des Hausarztes in sich vereint:

- Der Hausarzt als Arzt, der bestrebt ist, seinem ärztlichen Ethos zu folgen und sein ärztliches Selbstverständnis zu erfüllen.

- Der Hausarzt als Unternehmer, der mit seiner Praxis ein mittelständisches Unternehmen zu führen und zu erhalten hat.

- Der Hausarzt als Privatperson, die zeitliche Ressourcen für sich schaffen möchte.

Diese Rollenbilder geraten in Konflikt miteinander und sind Ausdruck der Zerrissenheit der Hausärzte. Es wird ein Gefühl des „Getriebenseins“ (CAO3) (CA06) beschrieben bei dem Versuch, den verschiedenen Rollen gerecht zu werden. Das Bestreben des Hausarztes, die bestmögliche medizinische Versorgung des Bewohners zu leisten, müsste eine höhere zeitliche Aufwendung bedeuten. Diese ist aber aus unternehmerischen Gesichtspunkten nicht leistbar und würde, wenn als idealistische und quasi unbezahlte Zusatzarbeit geleistet, die Privatperson hinter dem Arzt negativ beeinflussen. Hinzu kommt, als „intervenierende Belastung" und damit als Verstärkung des Konflikts, das bei CA09 beschriebene Anspruchsdenken:

„Ein ganz anderes wichtiges Problem, das ist das Zeitproblem, dass also die unser System und vor allem das System der Vergütung der ärztlichen Arbeit da nur eine recht minimale Versorgung zulässt, die möglicherweise ja ausreichend ist aber die nach den 
Kriterien die auch zum Beispiel Institutionen oder Kriterien die Angehörige an uns stellen wahrscheinlich dann doch wieder nicht ganz ausreichen." (CA09/34)

Das Durchführen von Hausbesuchen an sich wird als Teil des zu erfüllenden ärztlichen Ethos und Rollenbilds angesehen (CA09/55). Zusammenfassend wird die Problematik des Rollenkonflikts mit dem folgenden Zitat deutlich:

„....um den Mittelweg zu finden zwischen dem Leistbaren, was der Vergütung noch entspricht und aber dem Patienten vor allem gerecht zu werden." (CA08/119)

Die unternehmerischen bzw. ökonomischen Gesichtspunkte dieses Konflikts wurden ausführlich unter der ersten Fragestellung beschrieben. Das Bestreben nach bestmöglicher Patientenversorgung, wird durch das Rollenbild und ärztliche Selbstverständnis von Hausärzten nochmals deutlich. Das Problem des Zeitmangels ist allgegenwärtig und verhindert in seiner Konsequenz mehr persönliche Zuwendung und Geduld für den Bewohner. Dies würden sich Hausärzte jedoch wünschen, was die Zerrissenheit und den Rollenkonflikt verstärkt.

„Also man will es schon hören aber man darf es irgendwie nicht hören weil die Zeit einfach zu kurz ist ja. "(CA06/125)

„Na die Zeit des Arztes hat ja immer was damit zu tun, was für Qualitätsansprüche ich an meine eigene Arbeit lege und wie viel, wie wichtig mir ja sozusagen ärztlicherseits oder [...] von meiner Einstellung von meinem Ethos her sozusagen der Patient in einer Pflegeeinrichtung ist." (CAO3/46)

Die eigene Arbeitszufriedenheit trägt auch zur Qualität der Versorgung bei. Hieraus lässt sich abschließend erkennen, dass es für eine gute Versorgungsqualität von Heimbewohnern wichtig ist, die Arbeitsbedingungen für die versorgenden Hausärzte zu analysieren und zu optimieren.

„...verbessert das die Arbeitszufriedenheit? Denn die Arbeitszufriedenheit ist ganz, ganz wichtig, weil die Bewohnerinnen oder die 
Heiminsassen, wenn ich das mal so ganz überspitzt sage, die müssen das ja im Endeffekt ausbaden. "(AA07/315) 


\section{Diskussion}

\section{1. Übersicht über die wichtigsten Ergebnisse}

In dieser qualitativen Arbeit wurden drei Fragestellungen hinsichtlich der Wahrnehmungen und Bedürfnisse von 30 interviewten Hausärzten an drei Standorten untersucht. Die Wahrnehmungen und Bedürfnisse wurden 1) bezüglich der eigenen Arbeitssituation, 2) bezüglich der Versorgung und Betreuung der Bewohner und 3) hinsichtlich des Arbeitsumfelds Pflegeheim erörtert. Die ersten beiden Fragestellungen wurden zusammenfassend deskriptiv auf Basis des offenen Kodierens bearbeitet. Die dritte Fragestellung wurde zudem anhand des axialen und selektiven Kodierens exploriert. Hierbei konnte hinsichtlich der Wahrnehmung der Hausärzte „Zerrissenheit“ als zu Grunde liegendes zentrales Phänomen ermittelt werden. Diese Zerrissenheit resultiert aus dem sehr breiten, teilweise widersprüchlichen Spektrum von Aufgaben und Anforderungen, welches Hausärzte bei ihrer Arbeit im Heim wahrnehmen.

Es konnte gezeigt werden, dass die Arbeit im Heim für die interviewten Hausärzte einen grundlegenden Bestandteil ihrer hausärztlichen Tätigkeit darstellt und als bedeutsam für die dem demografischen Wandel ausgesetzte Bevölkerung wahrgenommen wird. Jedoch bestehen Hürden und Konfliktpotenzial: Die Hausärzte fühlen sich verschiedensten Anforderungen, wie der Vereinbarkeit von Praxistätigkeit, Arbeit im Heim und Privatleben und auch der Vereinbarkeit von gesellschaftlichen, gesetzlichen und moralischen Ansprüchen, ausgesetzt und erleben ein Gefühl der Zerrissenheit. Dies kann auf Dauer dazu beitragen, dass die Motivation und Bereitschaft der Arbeit im Heim gegenüber sinkt. Das Darlegen und Entschlüsseln dieser unterschiedlichen Anforderungen konnte mit dieser Arbeit zu großen Teilen erreicht werden.

Auf Bewohnerseite bestehen die Anforderungen aus Sicht der Hausärzte besonders in dem hohen Maß an Multimorbidität, dem Aushalten von seelischen und körperlichen Problemen, welche hausärztlich kaum zu lösen sind, und dem hohen (physischen wie psychischen) Versorgungsbedarf. Von Seiten der Pflegekräfte können mangelnde Kompetenz, zum Beispiel in der Umsetzung von ärztlichen Anordnungen oder dem korrekten Einschätzen von Gesundheitszu- 
ständen, Verfügbarkeit der Pflegekräfte und Probleme durch unterschiedliche Wahrnehmung von Notwendigkeiten und Interaktionswünschen als Bedingungen zur hausärztlichen Zerrissenheit beitragen. Für die Bewohner wünschen sich die Hausärzte mehr Zeit in der Versorgung von allen Beteiligten sowie mehr Individualität und Förderung von persönlichen Ressourcen in der Betreuung. Um mit ihrer Zerrissenheit umzugehen, wenden Hausärzte verschiedene Strategien an: Einige versuchen, der Zerrissenheit mit aktivem Verhalten und bewusster Ausgestaltung der Zusammenarbeit mit den Pflegekräften zu begegnen, andere reduzieren Zeit und Kontakt, sowohl zu den Pflegekräften als auch zu den Bewohnern. Darüber hinaus ist es für einige Hausärzte von besonderer Bedeutung, die eigenen Bedürfnisse wahrzunehmen und zu beachten, gepaart mit einer Strategie der Annahme der Situation. In Bezug auf die gesetzlichen Rahmenbedingungen besteht bei den Interviewpartnern besonders das Bedürfnis nach bedarfsgerechter und besserer Vergütung sowie nach Entlastung von bürokratischen Aufgaben. Auch das Bedürfnis nach mehr medizinischen Interventionsmöglichkeiten im Heim wird angesprochen. Die Entwicklung von tragfähigen Zukunftskonzepten, wie u. a. die Etablierung eines Heimarztes ist darüber hinaus ein Wunsch der Hausärzte.

Es soll abschließend erwähnt werden, dass viele Hausärzte nach wie vor Erfüllung und Freude an ihrer Tätigkeit im Heim finden, sei es durch die Zusammenarbeit im Team mit den Pflegekräften, die Abwechslung zur autonomen Praxistätigkeit oder durch die als meist positiv beschriebene Arzt-PatientenBeziehung.

\subsection{Stärken und Schwächen der Arbeit}

Die Stärken dieser Arbeit liegen besonders in der detaillierten und tiefgehenden Analyse der Wahrnehmungen und Bedürfnisse von Hausärzten hinsichtlich inrer Arbeit im Pflegeheim durch den qualitativen Forschungsansatz der Grounded Theory. Insbesondere zur dritten Forschungsfrage konnte ein umfangreiches Modell zur Erklärung der "Zerrissenheit“ erstellt werden, welches die aktuelle Sichtweise der Hausärzte widerspiegelt. Resultierend hieraus ergeben sich viele Ansatzpunkte, die Anlass für konkrete Veränderungen und Verbesserungen sein können. Eine Schwäche der Arbeit ist, dass das im qualitativen Rah- 
men angestrebte Erarbeiten der Ergebnisse durch die Zusammenarbeit von mehreren Forschern, um einen möglichst objektiven Standpunkt beizubehalten, nur in Teilen umgesetzt werden konnte, da die Analyse nahezu ausschließlich durch die Verfasserin der Arbeit durchgeführt wurde. Relativiert wird dies dadurch, dass sowohl die Konzipierung des Interviewleitfadens als auch die Interviewdurchführung und Teile des offenen Kodierens durch verschiedene Studienmitarbeiter erfolgten. Darüber hinaus wurde das Kodesystem und der Vorgang des axialen und selektiven Kodierens für die vorliegende Arbeit wiederholt und eng durch die Betreuerin Dr. med. C. Müller supervidiert. Begrenzungen entstanden auch durch das nur in geringen Teilen umsetzbare Theoretical Sampling bei der Interviewdurchführung. Letztlich war dieses jedoch für die Qualität der Ergebnisse nicht wesentlich, da bei der Auswertung inhaltlich eine Sättigung erreicht werden konnte.

\subsection{Einordnung innerhalb der themenbezogenen Literatur}

Die Ergebnisse dieser Arbeit weisen einige Übereinstimmungen, Überschneidungen und auch Ergänzungen zu der bestehenden Literatur auf. Beginnend soll hier auf die dritte Fragestellung eingegangen werden, die am differenziertesten bearbeitet wurde und den Fokus dieser Arbeit darstellt:

Wahrnehmungen des Arbeitsumfelds Pflegeheim - Das zentrale Phänomen der Zerrissenheit

Das zentrale Phänomen „Zerrissenheit" bei der Wahrnehmung des Arbeitsumfelds Pflegeheim aus Sicht der dort tätigen Hausärzte ist als solches in der bisherigen Literatur noch nicht beschrieben worden. Hausärzte erleben Zerrissenheit, wenn sie das Gefühl haben, den vielfachen Anforderungen und dem Wunsch, gute Arbeit leisten zu wollen, nicht gerecht werden zu können. Hier können Parallelen zu Studien gezogen werden, welche einen hohen Leistungsund Qualitätsdruck mit einer geringen Arbeitszufriedenheit assoziieren (Gothe 2010). Dies kann negativen Einfluss auf die Qualität der Versorgung und die Patientenzufriedenheit haben (van den Hombergh et al. 2009; Löffler et al. 2014). Auch die von den Ärzten wahrgenommene Qualität des Arzt-PatientenVerhältnisses korreliert mit der Gesamtzufriedenheit von Hausärzten. So zeigen 
Ärzte, die das Verhältnis zu ihren Patienten als gut bezeichnen, auch eine höhere Berufszufriedenheit (Löffler et al. 2014).

Ein hoher Leistungsdruck entsteht für die Hausärzte in unserer Studie unter anderem durch ihr eigenes Bestreben, sowohl dem hohen ärztlichen Anspruch an sich selbst als auch den unternehmerischen Aspekten einer Hausarzttätigkeit und den eigenen privaten Wünschen und Anforderungen gerecht zu werden. Hierin besteht ein wesentlicher Grund für die Zerrissenheit. Dieser Konflikt wird so auch in anderen medizinischen Bereichen zu finden sein können und scheint nicht (haus)arzt-spezifisch zu sein, wenngleich er auf Seiten der Hausärzte besonders deutlich zum Tragen zu kommen scheint. Über den aus den verschiedenen Anforderungen resultierenden Konflikt auf Hausarztseite wird jedoch auch in internationalen Studien berichtet (Hansson et al. 2007). Mehrere aus Deutschland stammende, vornehmlich quantitative Studien konnten zeigen, dass Hausärzte hinsichtlich Arbeitsbelastung und Zufriedenheit mit dem Umfang an zeitlichen Ressourcen für Familie und Freizeit eher unzufrieden sind (Schäfer et al. 2005; Schäfer et al. 2008; Goetz et al. 2011; Löffler et al. 2014) und somit ihre Rolle als Privatperson beziehungsweise ihre Work-Life-Balance nicht ausreichend erfüllt sehen. Dass die Rahmenbedingungen bei der hausärztlichen Tätigkeit im Heim, zum Beispiel durch das Erlangen eines allumfassenden Gesamtbildes des Bewohners, positive Chancen für die Hausärzte bieten können, wurde von anderen Autoren bereits beschrieben (Bolmsjö et al. 2015). Mit dieser Arbeit konnten weitere positive Aspekte gefunden werden, etwa die Möglichkeit für den Hausarzt, den Bewohner umfassend zu betreuen, ohne sich zu verausgaben, da die alltägliche Versorgung des Bewohners durch das Pflegeheimpersonal sichergestellt ist. Damit kann der betroffene Hausarzt die eigenen Bedürfnisse erfüllen. Als Konsequenz kann ein positives Erleben der Arbeit im Heim resultieren. Belastung und Betroffenheit bei der Tätigkeit im Heim können für viele Hausärzte als Konsequenz aus einer schlechten pflegerischen Versorgungssituation der Bewohner resultieren.

Die Zusammenarbeit mit den Pflegekräften prägt die Arbeit der Hausärzte im Heim wesentlich. In dieser Arbeit konnten viele Übereinstimmungen zu den bereits beschriebenen Einflüssen auf die Kooperation, wie Art und Qualität der 
Kommunikation, Kompetenzen der Pflegekräfte und Organisation der Pflegekräfte, gezeigt werden. Die Zufriedenheit mit der Zusammenarbeit wird dabei sowohl in der bestehenden Literatur als auch in unserer Studie als sehr inhomogen beschrieben. Zum einen kann dies mit der Ausprägung der beschriebenen Einflüsse erklärt werden, zum anderen können Hausärzte, die diese Einflüsse auf die Zusammenarbeit und die Art der Zusammenarbeit als intervenierende Bedingung wahrnehmen und daraus Strategien zur aktiven Gestaltung der Zusammenarbeit ableiten, eben diese verbessern und ihre Zerrissenheit reduzieren. Dies kann nicht nur auf den Bereich Hausarzt - Pflegeheim angewandt werden, sondern lässt sich auch auf andere Interaktionsbereiche des Gesundheitswesens übertragen.

Wie auch bei anderen Autoren dargestellt, gibt es verschiedene Bereiche, bei denen Hausärzte von der Arbeit und den Erfahrungen der Pflegekräfte profitieren (Meyer-Kühling et al. 2015). Jedoch erleben einige Hausärzte eine Erschwernis ihrer Tätigkeit durch fehlende Motivation und mangelnde Bereitschaft zur konstruktiven Zusammenarbeit durch die Pflegekräfte (Meyer-Kühling et al. 2015). Die Zusammenarbeit mit den Pflegekräften bewusst zu gestalten, kann für Hausärzte eine wichtige Strategie sein, um mit ihrer Zerrissenheit umzugehen. Es soll Erwähnung finden, dass einige Hausärzte bei ihren Vorstellungen zur Zusammenarbeit ein eher traditionelles Rollenbild zu vertreten scheinen. Dies spiegelt sich in Äußerungen der Hausärzte wider, die Pflegekräfte sollten dem Hausarzt helfen und zu einer Arbeitserleichterung des Hausarztes beitragen.

Laut unserer Ergebnisse stellt die Verfügbarkeit der Pflegekräfte für den Hausarztbesuch ein weiteres Feld oft kritischer Auseinandersetzungen dar. Auch von anderen Autoren wird dies als problematisch benannt (Meyer-Kühling et al. 2015). Es ist zu beachten, dass Pflegekräfte oft den Zeitpunkt des Hausbesuchs im Heim nicht kennen und sich somit auf den Hausbesuch oft nicht einstellen können. Die Erreichbarkeit der Pflegekräfte wird von unseren Interviewpartnern überwiegend bemängelt. Hausärzte empfinden das "Suchen“ nach einer zuständigen Pflegekraft als anstrengend. Feste Ansprechpartner im Heim und die Erreichbarkeit der Pflegekräfte werden auch von anderen Autoren 
als wichtige Faktoren für die Arbeit im Heim von Hausärzte gesehen (Tsakitzidis et al. 2017). In einer weiteren Arbeit aus dem INTERPROFStudienteam konnten Nina Fleischmann et al. "effektive Zusammenarbeit" als das zentrale Phänomen herausarbeiten (Fleischmann et al. 2016). Ebenfalls nach der Methode der Grounded Theory stellen Fleischmann et al. mit Hilfe des paradigmatischen Modells beeinflussende Faktoren, Strategien und Konsequenzen in Beziehung. Auch bei Fleischmann et al. wurde die Erreichbarkeit der Pflegekräfte als oftmals kritisch bezeichnet (Fleischmann et al. 2016). Der Fokus der genannten Arbeit lag auf der Interaktion von Hausärzten und Pflegekräften beim hausärztlichen Heimbesuch. Bolmsjö et al. erwähnen, dass der Wunsch nach mehr (pflegerischen) Ressourcen auch als Ausdruck angesehen werden kann, dass Hausärzte stetig gerne mehr für ihre Patienten tun würden, als es ihre Möglichkeiten zulassen (Bolmsjö et al. 2015). Dieser Wunsch und diese zugrundeliegende Motivation werden auch in der vorliegenden Arbeit mit der Strategie „Engagement" wahrgenommen. Zudem haben die hier interviewten Ärzte den Wunsch nach Kontinuität in der Zusammenarbeit, was auch der Perspektive von Ärzten in anderen Studien entspricht (Niehus et al. 2008; Meyer-Kühling et al. 2015). Die Reduzierung der Zusammenarbeit auf wenige feste Interaktionspartner wird auch in einem Kriterienkatalog für eine gelungene ärztliche Versorgung im Pflegeheim als sinnvoll erachtet (Karsch-Völk et al. 2015). Hier lassen sich Parallelen zu der von uns erhobenen Strategie der aktiven Gestaltung der Zusammenarbeit mit den Pflegekräften ziehen, wonach die Hausärzte auch versuchen, die Zusammenarbeit auf feste Interaktionspartner zu beschränken. Wie auch bei Karsch-Völk beschrieben, kann eine kontraproduktive Zusammenarbeit mit den Pflegekräften zur Konsequenz einer negativen Wahrnehmung des Arbeitsumfeldes führen, bis hin zur Empfindung des Pflegeheims als Spannungsfeld. Die Loslösung vom autonomen Arbeitsalltag in der Praxis und die Zusammenarbeit ähnlich einer Krankenhausvisite wird dagegen von den für diese Arbeit interviewten Ärzten als äußerst positiv empfunden, wie auch bei Bolmsjö et al. (Bolmsjö et al. 2015). 


\section{Wahrnehmung der Versorgung und Betreuung der Bewohner}

In den durchgeführten Interviews zeigten sich Sicherheit und Kompetenz in der Versorgung durch die Pflegekräfte als für die Hausärzte herausstechende Merkmale bezüglich der Versorgungssituation der Bewohner. Auch in der internationalen Literatur werden Verlässlichkeit und Kompetenz der Pflegekräfte als besonders wichtig erachtet (Bolmsjö et al. 2015; Kirsebom et al. 2016). Aus der hausärztlichen Perspektive ist dabei die korrekte Umsetzung von ärztlichen Anordnungen und die Medikamentenausgabe ein wichtiger Punkt. Hierzu besteht bei den von uns interviewten Hausärzten zum Teil große Unzufriedenheit. Über Missstände in der Verabreichung von Medikamenten wird auch in anderen Studien berichtet (Anliker et al. 2005). Unter anderem in diesem Bereich empfinden Hausärzte jedoch Abhängigkeit von den Pflegekräften (Bolmsjö et al. 2015). Dabei berichten Hausärzte auch über den Nutzen für die Sicherheit der Bewohner, wenn Pflegekräfte hier aufmerksam und kompetent agieren und zum Beispiel auf Allergien oder Unverträglichkeiten achten.

Ausreichend Zeit der Pflegekräfte, um hausärztliche Anordnungen umsetzen zu können, und um aktiv die Gesundheit der Bewohner zu fördern, beispielsweise durch Mobilisation, sehen die von uns interviewten Hausärzte als wichtigen Faktor, der auch die Möglichkeit der Prävention von Erkrankungen bietet. Dazu wünschen sich Hausärzte auch einen Ausbau an Beschäftigungsangeboten für die Bewohner. Ein „aktives Altern“ zu gestalten und zu ermöglichen, sollte Ziel aller an der Versorgung von Heimbewohnern Beteiligten sein. Dies zeigte sich als starkes Anliegen der Hausärzte in unseren Interviews. Andere Studien beschreiben, dass nach Einschätzung der Pflegekräfte knapp 60\% der Heimbewohner häufig oder gelegentlich niedergeschlagen oder antriebsgemindert seien (Schneekloth 2006). Im Kontext mit den hier vorliegenden Ergebnissen wird damit der erhobene Wunsch der Hausärzte nach Förderung des psychischen und physischen Wohlbefindens der Bewohner nochmals prägnanter. In diesem Zusammenhang wird auch die Notwendigkeit einer besseren personellen Aufstellung im Pflegeheim beschrieben, welche bereits von einigen Autoren dargestellt wurde (Oosterveld-Vlug et al. 2013; Kirsebom et al. 2016). Betrachtet man ergänzend Studien, die sich unter anderem mit der Entwicklung der personellen 
Strukturen in Pflegeberufen im Hinblick auf den demografischen Wandel beschäftigen, und die den Bedarf an professioneller Pflege schon zeitnah nicht mehr gedeckt sehen (Nowossadeck 2012), wird deutlich, dass die Umsetzung dieser Bedürfnisse und Wünsche schon allein durch solche äußeren Faktoren massiv erschwert wird. Ebenso gilt zu erwähnen, dass der Wunsch nach mehr Zeit für die eigentliche Versorgung wohl in den meisten Bereichen des Gesundheitswesens zu finden sein wird, und auch die Pflegekräfte dem Dilemma von wenig Zeit gegenüber hoher Arbeitsbelastung ausgesetzt sind.

Eine pflegerisch und medizinisch gesicherte Grundversorgung sollte laut den von uns interviewten Hausärzten Basis der Bewohnerversorgung im Heim sein. Über die Ausprägungen und Inhalte dieser "Grundversorgung" gibt es jedoch unterschiedliche Ansichten. Auch von Seiten der Kostenträger wird oftmals von einer derzeit bestehenden "gesicherten Grundversorgung" gesprochen und dies wird als Grundlage für eine Argumentation gegen weitere (finanzielle) Investitionen herangezogen. Für die von uns interviewten Hausärzte scheint die Sicherstellung von beispielsweise Körperpflege, Nahrungsaufnahme und Medikamentengabe jedoch die selbstverständliche Grundlage zu sein, auf der weitere Versorgungsaspekte aufbauen sollten. Des Weiteren zeigten sich in der Interviewauswertung die Förderung und Erhaltung von Individualität und ein respektvoller Umgang mit den Bewohnern als Anliegen und Bedürfnis aus Sicht der Hausärzte. Von anderen Autoren wird hierzu bemerkt: „Eine personzentrierte oder personorientierte Pflege gilt als Maßstab einer guten Pflege - nicht nur bei Menschen mit Demenz" (Bartholomeyczik und Halek 2017, S. 53). Autonomie, Fürsorglichkeit und die Förderung der Ich-Identität der Bewohner sind dabei wichtige Prinzipien (Bartholomeyczik und Halek 2017), besonders wenn man bedenkt, dass der Heimeinzug für die Bewohner oftmals mit der Angst vor Verlust der eigenen Unabhängigkeit und Konflikten im Bereich Autonomie und Identität verbunden sein kann (Riedl et al. 2013).

Insgesamt bestätigt sowohl die bestehende Literatur als auch die von uns durchgeführte Interviewanalyse, dass Hausärzte im Hinblick auf ihre Vorstellungen von der (optimalen) Versorgung der Bewohner und ihren Wünschen für die Bewohner das Pflegepersonal in einer besonders wichtige Position sehen 
(Bolmsjö et al. 2015; Kirsebom et al. 2016). Es bleibt zu diskutieren, ob diese Sichtweise auch darauf beruhen kann, dass Hausärzte ihre eigenen Handlungsmöglichkeiten möglicherweise als eingeschränkt wahrnehmen. Auch das Arzt-Patienten-Verhältnis wird in vielen Studien beleuchtet und als zentrales Motiv in der Berufsausübung des Hausarztes beschrieben (Wilm und in der Schmitten 2007). Im Vordergrund steht für die Hausärzte der Wunsch, ihre Patienten optimal versorgt zu wissen. Dies deckt sich mit Ergebnissen der bestehenden Literatur (Bolmsjö et al. 2015). Erfüllt sich dies, kann als Konsequenz ein positives Erleben der Arbeit im Heim entstehen.

\section{Wahrnehmung der eigenen Arbeitssituation und hausärztliche Wünsche}

Zur Wahrnehmung der eigenen Arbeitssituation befragt, äußerten sich die Hausärzte in unseren Interviews gehäuft zu den wirtschaftlichen Bedingungen ihrer Arbeit im Heim. Dabei zeigten sich die Hausärzte überwiegend kritisch und unzufrieden mit der bestehenden Situation und äußerten den Wunsch nach einer besseren finanziellen Vergütung der Heimtätigkeit. Auch in anderen Studien wird die finanzielle Vergütung als Hauptanliegen bzw. sogar als Hauptproblem beschrieben (Kalitzkus 2011). Die von uns interviewten Hausärzte sehen sich zum Teil in ihrer Tätigkeit wirtschaftlich benachteiligt und beklagen, dass die Versorgung von Heimbewohnern durch die Praxistätigkeit querfinanziert werden müsse. Die (wirtschaftlichen) Anreize, Heimpatienten zu betreuen, seien zu gering. Von einigen Hausärzten werden zudem die Themen Wirtschaftlichkeitsprüfung und Regressforderungen angesprochen und kritisiert. Die Belastung durch Regressforderungen wird auch von anderen Autoren belegt (Buddeberg-Fischer et al. 2007; Löffler et al. 2014). Für viele Hausärzte hängt die Qualität ihrer Arbeit eng mit der gegebenen Zeit für die Patientenversorgung und somit auch eng mit der finanziellen Vergütung zusammen. Es wird in den Interviews deutlich, dass sich Hausärzte eine Verbesserung der Vergütungssituation wünschen, um ihrem Anspruch von guter ärztlicher Praxis gerecht werden zu können. Zu diskutieren ist sicherlich, dass auch eine verbesserte Vergütungssituation nicht automatisch mehr Zeit schafft. Eine verbesserte und ausgebaute pflegerische Versorgung der Bewohner könnte die Hausärzte mit Sicherheit entlasten, muss jedoch auch von den Bewohnern selbst bzw. dem So- 
zialstaat finanzierbar sein. De facto mehr Zeit für die eigentliche Versorgung der Heimbewohner, sowohl aus ärztlicher als auch aus pflegerischer Sicht, könnte wohl durch den Abbau von administrativen und bürokratischen Aufgaben entstehen. Es gilt zu prüfen, an welchen Punkten dies möglich gemacht werden kann.

Bezüglich des Einflusses der Vergütung auf die generelle Berufszufriedenheit diskutieren andere Autoren recht unterschiedliche Ansichten. Hierbei gibt es Studienergebnisse, die den Einfluss der Vergütung auf die Berufszufriedenheit als eher gering einschätzen (Gothe 2010; Behmann et al. 2012), sowie Studien, die diesen Einfluss als sehr deutlich beschreiben (Löffler et al. 2014). Viele der von uns interviewten Ärzte geben an, dass durch bessere finanzielle Vergütung auch die Bereitschaft, Heimbesuche zu leisten, steigen könnte. Den Wunsch und die Legitimation für eine bessere Bezahlung begründen die Hausärzte zumeist damit, dass die multimorbide Kohorte der Heimbewohner einen höheren Versorgungsaufwand benötige und dies in den zeitlichen und finanziellen Ressourcen zu wenig Berücksichtigung fände. Außerdem seien die Besuche anstrengend und die Anfahrten zeitaufwendig und schlecht vergütet. Hier soll erwähnt werden, dass insbesondere die beiden letzten Punkte ebenso für Hausbesuche im Allgemeinen gelten.

Ein zusätzlicher Mehraufwand wird jedoch auch von anderen Autoren gesehen und laut diesen bei der Vergütung nicht hinreichend berücksichtigt (Kalitzkus 2011; Sharp 2011). Die beschriebenen empfundenen Belastungen und Herausforderungen durch die Arbeit im Heim werden auch in der nationalen (Sharp 2011; Krüger-Brand 2014) und internationalen Literatur (Katz et al. 2009; McDermott et al. 2012) berichtet. Weitere Autoren beschreiben den erhöhten Zeitaufwand, die Arbeitsbelastung und die Komplexität in der Betreuung von Heimpatienten (Groom et al. 2000; Corroon-Sweeney et al. 2009; Gleeson et al. 2014). In den genannten Studienergebnissen spiegeln sich die mit der vorliegenden Arbeit erhobenen Konsequenzen aus dem Phänomen der Zerrissenheit (Hilflosigkeit und Deprimiertheit sowie Überforderung und Belastung) wider. Es gilt zu hoffen, dass durch die inzwischen getroffenen Regelungen und Vergütungspauschalen, basierend auf Kooperationsverträgen zwischen Ärzten und 
Pflegeheimen (Kassenärztliche Bundesvereinigung 2016), Abhilfe bezüglich der Unzufriedenheit mit der Vergütung geschaffen werden kann.

Hinsichtlich der gesetzlichen Rahmenbedingungen wünschen sich die von uns interviewten Hausärzte eine Entlastung von bürokratischen und dokumentarischen Aufgaben, insbesondere da in diesem Bereich eine Zunahme der Anforderungen verzeichnet wird. Unsere Ergebnisse decken sich mit anderen Studien, welche eine hohe Unzufriedenheit mit dem Umfang der bürokratischen Aufgaben und der Dokumentationslast beschreiben (Schäfer et al. 2008; Kalitzkus 2011; Koch et al. 2011; Behmann et al. 2012). Die hieraus resultierende Zerrissenheit, der Wunsch nach mehr Zeit für die eigentliche Versorgung einerseits und die Verpflichtung zur Erfüllung bürokratischer Aufgaben andererseits, sind sicherlich auch in anderen Sparten des Arztberufes zu finden und werden allgemein eher als Negativtrend von den Betroffenen wahrgenommen. Ein weiterer Wunsch der interviewten Hausärzte ist eine höhere gesellschaftliche Wertschätzung. Diesbezüglich beschreiben andere Studien einen Negativtrend, also eine Abnahme an Ansehen und Wertschätzung der hausärztlichen Profession (Natanzon et al. 2010). Zu erwähnen ist, dass frühere Studien das empfundene Ansehen als eher über dem Durchschnitt liegend beschreiben (Schäfer et al. 2005).

Die durchgeführten Interviews zeigen, dass die Zusammenarbeit mit den Heimen und hier insbesondere mit den Pflegekräften die Wahrnehmung der Arbeitsbedingungen im Heim durch die Hausärzte massiv beeinflusst. Die bestehende Literatur gibt zur Art der Zusammenarbeit ein sehr unterschiedliches Bild wieder. Als positiv werden Wertschätzung und das Profitieren und Lernen von den Pflegekräften erlebt (Meyer-Kühling et al. 2015). Negativ dagegen wird über Auseinandersetzungen zu Verantwortlichkeiten und Verordnungen (Kalitzkus 2005) und über aus Hausarztsicht unnötige Besuchsanforderungen (Anliker et al. 2005; Krüger-Brand 2014) berichtet. Diese Besuchsanforderungen liegen laut den interviewten Hausärzten besonders in dem wahrgenommenen Absicherungsbedürfnis der Pflegekräfte begründet. Das aktive Erkennen und Mitteilen von Auffälligkeiten durch die Pflegekräfte ist für den Hausarzt essentiell, um adäquat auf die Bedürfnisse der Bewohner eingehen zu können. 
Diese Erwartung teilen auch die von Anliker et al. befragten Hausärzte (Anliker et al. 2005). Besonders, da die Initiierung der Heimbesuche in den meisten Fällen über die Pflegekräfte erfolgt. Hausärzte können hierbei von qualifizierten Pflegekräften, welche Gesundheitszustände kompetent einschätzen können, stark profitieren. Gerade diesen Bereich betreffend wird von vielen der interviewten Hausärzte jedoch großes Verbesserungspotenzial gesehen.

Besonders beim Heimbesuch selbst ist eine gute Zusammenarbeit von Hausärzten und Pflegekräften für die Qualität der Versorgung der Bewohner essentiell. Die qualitative Studie von Tsakitzidis et al. kommt zu dem Schluss, dass die interprofessionelle Zusammenarbeit im Pflegeheim (in Belgien) noch nicht ausreichend etabliert ist (Tsakitzidis et al. 2017). Der Wunsch nach einer guten Zusammenarbeit besteht sowohl bei den von uns interviewten Hausärzten als auch laut den Ergebnissen anderer Autoren (Anliker et al. 2005). Einige der von uns interviewten Hausärzte wünschen sich von den Pflegekräften, dass diese den Heimbesuch vorbereiten. Ob eine Begleitung der Pflegekräfte während des Bewohnerkontakts selbst stattfinden soll, darüber sind die von uns interviewten Hausärzte sehr unterschiedlicher Auffassung. Der überwiegende Teil der Hausärzte in unseren Interviews wünscht sich eine engere Zusammenarbeit mit den Pflegekräften. Beinhalten soll dies auch einen angemessenen und konstruktiven Austausch von Kritik und Informationen. Interprofessionelle Fallbesprechungen werden hierbei als Möglichkeit der praktischen Umsetzung genannt. Dieser Bedarf wird auch von anderen Autoren beschrieben (MeyerKühling et al. 2015).

Einige Hausärzte wünschen sich mehr diagnostische und therapeutische Möglichkeiten im Heim. Als Beispiele werden bei uns und in der Literatur die Möglichkeiten zur intravenösen Therapie und Labordiagnostik genannt (Kirsebom et al. 2016). Hierzu überleitend wird in unseren Interviews auch über das Konzept des „Heimarztes" diskutiert. Hierbei gehen die Ansichten und Auslegungen zu einem solchen Konzept auseinander. Vorteile werden unter anderem in der Kontinuität, Vertrautheit und in einer möglichen Reduzierung von Krankenhauseinweisungen gesehen. Als problematisch wird die dann nicht mehr freie Arztwahl zu Bedenken gegeben sowie die mögliche Einseitigkeit einer solchen 
Tätigkeit. Darüber hinaus bezweifeln einige Hausärzte einen zusätzlichen Nutzen durch eine stärkere Präsenz des Hausarztes im Heim. Weitere Wünsche von Hausärzten sind die Schaffung von regelmäßigen Austauschforen der an der Versorgung von Pflegeheimbewohnern Beteiligten. Diese sollen die Möglichkeit eines gegenseitigen Austausches zur Verbesserung der Versorgungsqualität von Heimbewohnern bezwecken. Auch internationale Forscher beschreiben Wünsche und Bedürfnisse dieser Art (McDermott et al. 2012).

\subsection{Schlussfolgerung}

Mit dieser qualitativen Arbeit über die Wahrnehmungen und Bedürfnisse von Hausärzten hinsichtlich ihrer Tätigkeit im Pflegeheim wird ein Beitrag für ein tieferes Verständnis und zu einem umfangreicheren Bild der hausärztlichen Sichtweise geleistet. Das sich ergebende zentrale Phänomen der Zerrissenheit bietet Ansatzpunkte, um Verbesserungen für den hausärztlichen Tätigkeitsbereich im Pflegeheim zu schaffen.

Wünschenswert wäre es, wenn die vorliegende Arbeit dazu beitragen konnte, die Wahrnehmung der Hausärzte zu beleuchten, das emotionale Erleben und die Bedürfnisse der Hausärzte als wichtige Faktoren hervorzuheben und diese für weitere Forschungen und Verbesserungen auf dem Gebiet der Pflegeheime Deutschlands in den Fokus zu rücken. Denn vor dem Hintergrund des demografischen Wandels und der Zunahme an Bewohnern in der stationären Altenpflege stellen Hausärzte eine wesentliche Säule in der medizinischen Versorgung von Pflegeheimbewohnern dar. Wenn die Hausärzte mit ihren Arbeitsbedingungen zufrieden sind und sich in ihren Bedürfnissen ernst genommen fühlen, trägt dies zur Sicherung der medizinischen Versorgung der Bewohner bei.

Die Vereinbarkeit der verschiedenen hausärztlichen Rollen zu fördern und die Anforderungen ausgehend von den Bewohnern, den Pflegekräften und von Instanzen wie der Kassenärztlichen Vereinigung zu bündeln und zu minimieren, sollten Ziele weiterer Verbesserungen auf dem Gebiet der hausärztlichen Heimtätigkeit sein.

Das Erleben des Settings Pflegeheim ist bei vielen Hausärzten emotional negativ besetzt. Da davon auszugehen ist, dass die Grundhaltung und das Empfin- 
den dem eigenen Arbeitsumfeld gegenüber wichtige Faktoren auch für die Gesamtzufriedenheit sein könnten, wäre eine stärkere Berücksichtigung dieses Gebiets für zukünftige Forschungen wünschens- und erstrebenswert. Es ist anzunehmen, dass eine Reduktion der von den Hausärzten dieser Studie wahrgenommenen Zerrissenheit zur Verbesserung der eher negativen und ablehnenden Haltung der Hausärzte gegenüber dem Tätigkeitsfeld Pflegeheim beitragen könnte.

Die mit dieser Arbeit eruierten Strategien der Hausärzte, etwa das Achten auf eigene Bedürfnisse, Engagement oder auch aktive Gestaltung der Zusammenarbeit mit den Pflegekräften, können genutzt werden, um daraus allgemein anwendbare Handlungsmöglichkeiten abzuleiten. Diese könnten bereits im Medizinstudium und der hausärztlichen Weiterbildung sowie auf Fortbildungsveranstaltungen angesprochen werden. Durch verbesserte Rahmenbedingungen, besonders den Abbau von bürokratischen Anforderungen und Aufgaben, könnte weiterhin zur Reduktion der Zerrissenheit beigetragen werden. Es ist zu erwähnen, dass mit dem Pflegeneuausrichtungsgesetz von 2012 bereits Anreize geschaffen werden sollten, die hausärztliche Arbeit im Heim zu fördern (Bundesministerium für Gesundheit 2012). Eine umfassende Umsetzung der dort angestrebten Kooperationsverträge zwischen Pflegeheimen und (Haus-) Ärzten erfolgte jedoch nicht. Seit Juli 2016 ist es jedoch durch die Erweiterung des Einheitlichen Bewertungsmaßstabes um das Kapitel 37 möglich, eine zusätzliche Vergütung für die Versorgung von Pflegeheimpatienten zu erlangen (Kassenärztliche Bundesvereinigung 2016; Kassenärztliche Bundesvereinigung 2018). Hausärzte, die auf Grundlage des $§ 119 b$ SGB V Kooperationsverträge mit den Pflegeheimen schließen, können Zuschläge geltend machen. Mit den zusätzlichen Vergütungspauschalen wird der Mehraufwand nun auch entsprechend finanziell honoriert. Es bleibt zu hoffen, dass diese Veränderung in der Vergütung die Kooperation von Hausärzten und Pflegeheimen flächendeckend stärken kann und zu einer verbesserten Versorgung der Bewohner führt.

Es ist weiterhin zu diskutieren, ob die Arbeit der Hausärzte im Heim erleichtert werden könnte, wenn mehr diagnostische Möglichkeiten oder auch mehr Interventionsmöglichkeiten im Pflegeheim geschaffen würden. Dies käme den Be- 
wohnern zu Gute, wenn dadurch beispielsweise Krankenhauseinweisungen reduziert werden könnten. Die (knappen) zeitlichen Ressourcen, von Ärzten und Pflegepersonal, würden gleichzeitig jedoch zunehmend beansprucht. Ebenso bleibt die Frage der Kostenübernahme zu klären. Auch über eine Innovation der hausärztlichen Versorgung im Heim, beispielsweise durch die Etablierung von Heimärzten, sollte beraten werden. Ansätze und Anregungen konnten mit der vorliegenden Arbeit geschaffen werden.

Die Qualifikation und Kompetenz der Pflegekräfte, beispielsweise in den Bereichen der sicheren Umsetzung von ärztlichen Anordnungen, der Einschätzung von Gesundheitszuständen und, auf Basis dessen, der bedarfsgerechten Initiierung von Hausarztbesuchen, sind für die Hausärzte im Heim besonders wichtig und können eine Unterstützung der ärztlichen Tätigkeit sein. Pflegekräfte sollten, auch um ihre eigene Selbstständigkeit und ihr Selbstbewusstsein zu stärken, in diesen Bereichen weiter gefördert werden. Ebenso sollte die Ausbildung und der Einsatz von Fachkräften weiter gefördert werden. Schulungen, Fortbildungen und stärkere praktische Fokussierung in der Ausbildung können hier Ansatzpunkte sein. Auch eine Vertiefung der interprofessionellen Zusammenarbeit und der interprofessionellen Ausbildung sollte bedacht werden. Durch gemeinsamen Unterricht von Auszubildenden in Pflegeberufen und Medizinstudierenden können Verständnis und Wahrnehmung der gegenseitigen Sichtweisen erreicht werden, was später in gemeinsamen Fortbildungen vertieft werden könnte. Ein weiterer Lösungsansatz könnte in verbindlichen Absprachen von Hausärzten und Pflegekräften liegen, damit sich die Pflegekräfte auf den Hausbesuch einstellen und Vorbereitungen treffen können, besonders da Hausärzte die Reduzierung der Wartezeiten im Heim als Bedürfnis die Zusammenarbeit betreffend nennen.

Die Versorgungssituation der Heimbewohner ist letztlich maßgeblich für das Empfinden der Hausärzte dem Heim gegenüber. Die Individualität und Autonomie der Heimbewohner könnte durch mehr Angebote und Zeit in der Versorgung unterstützt werden. Wenn hierbei der Fokus auf individualisierter Bedürfniserfüllung der Bewohner liegen würde, könnte auch der hausärztliche Ein- 
druck vom Heim positiver ausfallen. Übergeordnetes Ziel bleibt stets, eine gute Versorgung der Pflegeheimbewohner auch in Zukunft gewährleisten zu können. 


\section{Zusammenfassung}

Hintergrund: Hausärzte stellen eine wesentliche Säule in der Versorgung von Pflegeheimbewohnern dar. Ein differenziertes Verständnis der ärztlichen Perspektive kann helfen, verbesserte Bedingungen für die im Pflegeheim tätigen Hausärzte zu schaffen und damit die Versorgung von Pflegeheimbewohnern in Deutschland zu stärken.

Fragestellung: Ziel der Arbeit war es, die Wahrnehmungen und Bedürfnisse von Hausärzten hinsichtlich ihrer Tätigkeit im Pflegeheim abzubilden. Hierzu wurden drei Fragestellungen betreffend die Wahrnehmungen und Bedürfnisse hinsichtlich 1) der eigenen Arbeitssituation, 2) der Versorgung und Betreuung der Bewohner und 3) des Arbeitsumfelds im Pflegeheim entwickelt.

Methode: Von August 2012 bis August 2013 wurden in drei verschiedenen Städten Deutschlands (Göttingen, Hamburg, Mannheim) offene Leitfadeninterviews mit 30 an der hausärztlichen Versorgung von Pflegeheimbewohnern beteiligten Ärzten geführt. Anschließend wurden die Interviews transkribiert, pseudonymisiert und nach der Methode der Grounded Theory ausgewertet. Das Interviewmaterial wurde für diese Arbeit auf die drei Fragestellungen hin untersucht. Für Frage eins und zwei wurde ein zusammenfassend deskriptives, auf Basis des offenen Kodierens basierendes Vorgehen gewählt. Für Frage drei folgte eine detailliertere Analyse und Darstellung nach allen CodierSchritten der Grounded Theory.

Ergebnisse: Als zentrales Phänomen, die Arbeit der Hausärzte im Heim betreffend, konnte „Zerrissenheit“ identifiziert werden. Hausärzte haben den übergeordneten Wunsch, gute Arbeit zu leisten und den bestehenden Anforderungen gerecht zu werden. Die Anforderungen durch die in der Betreuung aufwendigen Heimbewohner, die Zusammenarbeit mit den Pflegekräften und die gesellschaftlichen und gesetzlichen Rahmenbedingungen sind für die Hausärzte prägend und tragen zur Zerrissenheit bei. Hausärzte können dabei einen Rollenkonflikt ihre Person als Arzt, Unternehmer und Privatperson betreffend empfinden. Die Kompetenzen der Pflegekräfte, beispielsweise in der korrekten Einschätzung von Gesundheitszuständen, dem Verhalten in Akutsituationen aber 
auch im Umgang mit den Bewohnern sind für die Wahrnehmung der Hausärzte bezüglich des Arbeitsumfelds Pflegeheim sehr bedeutsam. Für die Bewohner wünschen sich die Hausärzte mehr Zeit in der Versorgung, ein würdevolles Altern und die Förderung von körperlichen und kognitiven Fähigkeiten. Zwar berichteten einige Hausärzte über Freude an der Arbeit im Heim und über ein positives Erleben die Arbeit im Team mit den Pflegekräften betreffend. Das Arbeitsumfeld Pflegeheim wurde von den interviewten Hausärzten jedoch als emotional überwiegend negativ besetzt beschrieben. Überforderung und Belastung durch die Ballung von multimorbiden Menschen, deren Bedürfnisse teils nicht ausreichend erfüllt werden können, trugen hierzu bei. Die bürokratischen Anforderungen und die Vergütungssituation waren darüber hinaus für die meisten Hausärzte wichtige und kritisch beurteilte Faktoren die Arbeit im Heim betreffend.

Schlussfolgerung: Um die Versorgung von Pflegeheimbewohnern zu stärken, ist die Berücksichtigung der Perspektive der beteiligten Akteure über die aktuelle Situation und somit auch die Kenntnis über die Sichtweise der Hausärzte auf ihre Arbeit im Pflegeheim wesentlich. Die aktuell vielfältigen Anforderungen an die Hausärzte tragen zu einem Gefühl der Zerrissenheit für ihre Arbeit im Pflegeheim bei. Um die Motivation zur Arbeit im Heim auch für die Zukunft zu stärken und die Wahrnehmung der Hausärzte in ein positiveres Licht zu rücken, wäre es von Nutzen, für eine verbesserte Vereinbarkeit der Anforderungsbereiche Sorge zu tragen.

Die Qualifikation und Kompetenz der Pflegekräfte sind für die Hausärzte im Heim besonders wichtig und können eine Unterstützung der ärztlichen Tätigkeit sein. Es sollte daher versucht werden, Pflegekräfte in diesen Bereichen weiter zu fördern. Die als kritisch gesehenen Punkte, wie bürokratischer Aufwand und Vergütung, sollten Ansatzpunkte weiterer Verbesserungen sein. Zuletzt könnten die Rahmenbedingungen, wie die Ausgestaltung eines Heimarztkonzepts oder die Möglichkeit zu mehr Diagnostik und Intervention im Heim, auf Grundlage der beschriebenen Wahrnehmungen und Bedürfnisse der Hausärzte konkretisiert werden. 
Es bleibt zu hoffen, dass die Position der Hausärzte in der Heimversorgung gesehen und gestärkt wird. Vor dem Hintergrund des demografischen Wandels sind für den Bereich der heimärztlichen Tätigkeit sinnvolle Konzepte und Veränderungen, basierend auf den Wahrnehmungen und Bedürfnissen der Hausärzte, wesentlich, um eine gute Versorgung der Pflegeheimbewohner zu gewährleisten. 


\subsection{Summary}

Introduction: General practitioners (GPs) are an essential element in the care of nursing home residents. A differentiated understanding of the medical perspective can help to create improved conditions for GPs working in nursing homes and thus strengthen the care of nursing home residents in Germany.

Questions: The aim of this paper was to illustrate the perceptions and needs of GPs with regard to their work in nursing homes. For this purpose, three research questions were developed. They concerned perceptions and needs with regard to 1) GPs' own work situation, 2) the support and care of residents and 3 ) the working environment in nursing homes.

Methods: From August 2012 to August 2013, open guided interviews were conducted in three different cities in Germany (Göttingen, Hamburg, Mannheim) with 30 physicians involved in the provision of medical care to nursing home residents. The interviews were then transcribed, pseudonymised and evaluated according to the Grounded Theory method. For this thesis, the gained data was examined with regard to the three research questions. For questions one and two, a summary descriptive procedure based on open coding was chosen. In case of question three, a more detailed description following all coding steps of the Grounded Theory method was selected.

Results: As the central phenomenon concerning the work of GPs in nursing homes, "inner strife" could be identified. GPs have the overriding desire to do good work and to fulfill the existing demands. The demands of home residents, the cooperation with the nursing staff and the social and legal framework conditions are influential for GPs and contribute to the inner strife. GPs can feel that a conflict of roles affects them as physicians, businessmen and private individuals. The skills of the nursing staff, for example in the correct assessment of health conditions, the behaviour in acute situations and in dealing with the residents, are very important for the GPs perception of the nursing home working environment. For the residents, the GPs would like to have more time in care, dignified aging and the promotion of physical and cognitive abilities. Some GPs reported about the joy of working in nursing homes and about a positive experience concerning the work in the team with the nursing staff. However, in the interviews, the working environment in the nursing home was described by the GPs as emotionally predominantly negative. Overstrain and stress caused by the concentration of multimorbid people, some of whose needs could not be sufficiently fulfilled, contributed to this. In addition, the administrative require- 
ments and the reward situation were important and critically assessed factors for most GPs regarding work in nursing homes.

Conclusions: In order to strengthen the care of nursing home residents, it is essential to consider the perspective of the actors involved about the current situation and therefore also the knowledge of GPs' view on their work in nursing homes.

The many and varied demands placed on GPs today contribute to a feeling of inner strife for their work in the nursing home. In order to strengthen the motivation to work in nursing homes for the future and to place the view of GPs in a more positive light, it would be useful to ensure a better compatibility of the different requirements.

The qualification and competence of the nursing staff are particularly important for the GPs in the nursing home and can support the work of the physicians. Nurses should be further encouraged in these areas, also in order to strengthen their own independence and self-confidence. Critical points such as administrative burdens and rewards should be the starting points for further improvements. Finally, the framework conditions, such as the design of a home doctor's concept or the possibility of more diagnostics and interventions in the nursing home could be specified on the basis of the perceptions and needs of the GPs described above.

It is to be hoped that the position of GPs in nursing home care will be seen and strengthened. Considering the demographic change, meaningful concepts and changes in the field of nursing home medicine, based on the perceptions and needs of GPs, are essential to ensure good care for nursing home residents. 


\section{Anhang}

Anhang a Anschreiben Hausärzte

\section{UniversitätSMEDIZIN :
GÓtTINGEN : $\mathbf{M G}$}

Universitätsmedizin Göttingen, 37099 Göttingen

Direktorin des Instituts für Allgemeinmedizin
Prof. Dr. Eva Hummers-Pradier, Humboldtallee 38

Dr. med

$\mathrm{XX}$

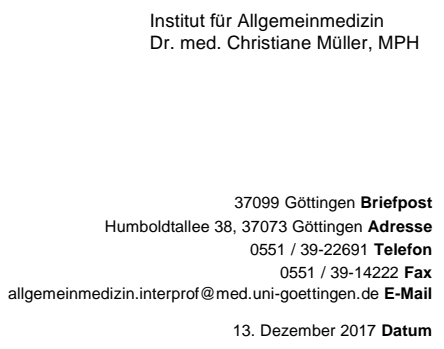

Institut für Allgemeinmedizin

13. Dezember 2017 Datum

Bitte um Unterstützung der Studie „interprof“

„Interprofessionelle Zusammenarbeit und Kommunikation im Pflegeheim“

Sehr geehrter Herr Dr. XX,

betreuen Sie als Hausarzt Patientinnen und Patienten, die in einem Alten- oder Pflegeheim leben? Ihre Erfahrungen in der Zusammenarbeit mit Pflegeheimen interessieren uns sehr!

Wir bitten Sie um Ihre Mitarbeit an der vom Bundesministerium für Bildung und Forschung geförderten Studie "interprof".

Die Studie untersucht die Zusammenarbeit und Kommunikation zwischen verschiedenen Berufsgruppen im Pflegeheim. Darüber hinaus wollen wir mehr über die Sichtweisen der Ärzte, der Pflege sowie der Bewohner und ihrer Familien erfahren. Wir würden gerne mit Ihnen innerhalb der nächsten Monate einen Termin für ein persönliches Gespräch (Interview) vereinbaren. In diesem geht es um Ihre Perspektive und Ihre Erfahrungen bei der Zusammenarbeit mit Pflegeheimen und Pflegepersonal. Das Interview dauert ca. 60 Minuten, Ort und Zeitpunkt bestimmen Sie. Für Ihren Aufwand erhalten Sie eine Entschädigung in Höhe von 50 Euro.

Gerne rufen wir Sie in den nächsten Tagen an, um Ihre Teilnahmebereitschaft zu erfragen; oder Sie senden das beiliegende Antwortblatt per Fax oder Post an uns zurück. Eventuelle Fragen beantwortet Dr. Christiane Müller gerne telefonisch (0551-3922691). Mit Ihrer Teilnahme an der Studie leisten Sie einen wertvollen Beitrag zur allgemeinmedizinischen Forschung. Wir bedanken uns im Voraus für Ihr freundliches Interesse und würden uns über eine zustimmende Rückmeldung sehr freuen!

Mit besten Grüßen

Ihre

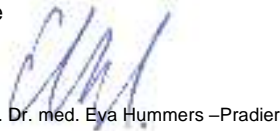

Dr. med. Christiane Müller, MPH

Projektleitung

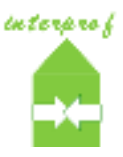


Dr. med. Christiane Müller

Humboldtallee 38

37073 Göttingen

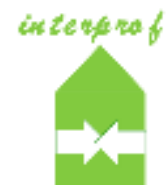

\section{$\square$ JA,}

ich bin an einer Teilnahme am Forschungsprojekt „,interprof, interessiert.

Dieser Zeitraum wäre für ein Interview besonders günstig:

\section{$\square$ NEIN,}

eine Studienteilnahme kommt für mich nicht in Frage 
Anhang $b$ Informationsschreiben

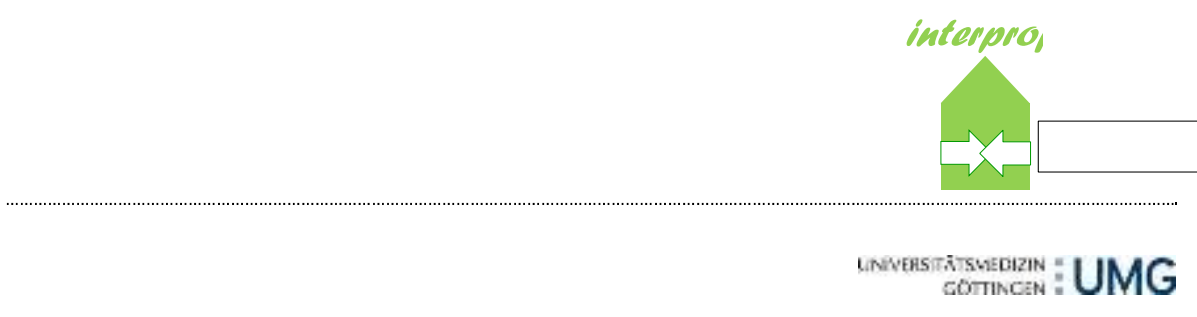

„Interprofessionelle Zusammenarbeit und Kommunikation im Pflegeheim“ 


\title{
interprof \\ Interprofessionelle Zusammenarbeit und Kommunikation im Pflegeheim \\ Projekteitung: \\ Prof. Dr. med. Eva Hummers-Pradier und Dr. med. Christiane Müller, MPH Institut für Allgemeinmedizin der Universitätsmedizin Göttingen \\ Humboldtallee 38 \\ D-37073 Göttingen

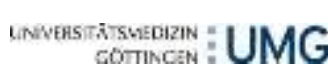

Einverständniserklärung zur Teilnahme an der Studie „Interprofessionelle Zusammenarbeit und Kommunikation im Pflegeheim“ (Interview)

\begin{abstract}
Ich wurde über die o. g. Studie von einer Studienmitarbeiterin vollständig aufgeklärt und habe den Informationstext gelesen und verstanden. Ich hatte die Gelegenheit, Fragen dazu zu stellen. Die Antworten habe ich verstanden und akzeptiert. Ich hatte genügend Zeit, mich zur Teilnahme $z u$ entscheiden. Ich weiß, dass die Teilnahme freiwillig und mit keinem unmittelbaren persönlichen Nutzen oder Nachteilen für mich verbunden ist. Ich weiß, dass ich jederzeit und ohne Angabe von Gründen diese Zustimmung widerrufen kann, ohne dass dadurch Nachteile für mich entstehen. Alle bis dahin erhobenen Daten, sowie die Audioaufnahmen im Original und der DVD als Sicherheitskopie würden in diesem Fall unverzüglich vernichtet werden.

Ich bin damit einverstanden, dass die im Rahmen der Studie erhobenen Daten und Audiodaten pseudonymisiert unter den Bedingungen des Datenschutzes für einen Zeitraum von 10 Jahren gespeichert und zu Forschungszwecken verwendet werden. Die personenbezogenen Daten werden nicht an Dritte weitergegeben.

Ich bin weiterhin damit einverstanden, dass das Interview auf Tonband aufgezeichnet wird.
\end{abstract}

Ich habe den Informationstext und eine Kopie dieser Einwilligung erhalten.

Vor- und Nachname Teilnehmerln

Ort, Datum, Unterschrift Teilnehmerln

Ort, Datum, Unterschrift Studienmitarbeiterln 
Anhang d Interviewleitfaden

Themenber eiche für die offenen Leitfadeninterviews Arzt

31.07.2013

\section{Erzählung einestypischen Hausbesuchs im Pflegeheim}

Wenn Sie einmal so zurückdenken, wie verläuft denn einer I hrer Besuche im Pflegeheim üblicherweise ab?

Wann und wie werden Sie konsultiert? Was gehört zu Ihren Tätigkeiten?

(Wie läuft es, wenn neue Medikamente verschrieben werden, wie ist da der Weg? Erzählen Sie)

Wie erleben Sie die Kommunikation mit den Bewohnern?

Wie verlaufen die Kontakte zu den Angehörigen in der Regel?

Kontinuität in der Versorgung von Patienten ist ja auch ein Thema im Pflegeheim, wie ist es denn bei Ihnen? (Werden Ihre Patienten beispielsweise noch von anderen Ärzten betreut? Erzählen Sie...)

Wenn Sie an Ihre hausär đlliche Tätigkeit im Heim denken, welche positive/negative Erfahrungen haben Sie da schon gemacht? Nennen Sie gerne einige Beispiele. Zum Bsp. hinsichtlich der Rahmenbedingungen, der Organisation von Abläufen, bei den Zuständigkeiten im Team? Erzähl en Sie...

\section{Beschreibung des letzten Hausarztbesuchs}

Können Sie sich noch an I hren letzten Hausbesuch bei einem Pflegeheimbewohner erinnern? Wie war das denn so in diesem konkreten Fall? Schildern Sie doch mal den genauen Ablauf.

Wie kam es dazu, dass Siekonsultiert wurden?

Wie verlief dann der Besuch bei Ihrem Patienten?

(Hatten Sie beim letzten Hausbesuch Kontakt zum Pflegepersonal oder zu den Angehörigen des Patienten?)

Haben Sie nach dem letzten Hausbesuch den Gesundheitszustand I hres Patienten verfolgt? Also wie ging es dann weiter?

3. Erfahrungen mit Zuständigkeitsbereichen und Aufgabenverteilungen

Uns interessiert, wie Aufgaben im Pflegeheim verteilt sind. Welche Aufgaben übernehmen Sie und für was ist das Pflegepersonal zuständig? 
Vielleicht können Se sich auch an einzelne Stuationen erinnern die Ihnen in Bezug auf die Aufgabenverteilung positiv oder negativ aufgefallen sind?! Erzählen sie..

(Welche Aufgaben übernehmen die Bewohner denn noch so selbst?)

Welche pflegerischen Aufgaben oder Tätigkeiten sind in Ihren Augen besonders wichtig?

Sehen Pflegende manchmal Stuationen in der medizinischen Versorgung anders als Sie?

(Gibt es auch Aufgaben für die die Angehörigen zuständig sind?)

Was glauben Sie macht einen guten Hausarzt im Heim aus?bzw. immanent Nachfragen bei Selbstbild

4. Vorstellungen und Phantasien über die ideale Situation/Versorgung im Pflegeheim

Lassen Sie I hrer Phantasie freien Lauf. Waswäre für Sie die ideale hausärztliche Versorgung im Pflegeheim? Waswürden Sie sich da wünschen?

Was würden Sie sich für die medizinische Versorgung wünschen, falls I hre Eltern ins Pflegeheim kämen?

Wie sähe in Ihren Augen die optimale Zusammenarbeit mit den Pflegekräften aus, wenn Sie sich über Zeit, Geld, Ort keine Gedanken machen müssten....?

Was wäre davon im aktuellen Rahmen umsetzbar, oder haben Sie andere konkrete Verbesserungsvorschläge...

Wie sähe in Ihren Augen die ideale Aufgabenteilung zwischen den Ärzten und dem Pflegepersonal aus?

Momentan wird in der Berufspolitik ja viel über die Übernahme von är ztlichen Tätigkeiten durch die Pflege diskutiert. Welche Aufgaben könnten Sie sich vorstellen, an Pflegekräfte komplett abzugeben. Dies bedeutet, dass die Anordnungs-und Durchführungsverantwortung nur noch bei der Pflege läge? Welche Tätigkeiten wären dies?

Was denken Sie, was wünschen sich die Pflegekräfte? Welche Abläufe wär en für die Pflegekräfte wohl optimal? Was denken Sie?

Und die Bewohner? Was wünschen die sich wahrscheinlich? 


\section{Literaturverzeichnis}

Andrew RA (1988): Analysis of a general practitioner's work in a private nursing home for the elderly. J R Coll Gen Pract $\underline{38}, 546-548$

Angerer P, Schwartz FW: Einführung. In: Schwartz FW, Angerer P (Hrsg.): Arbeitsbedingungen und Befinden von Ärztinnen und Ärzten: Befunde und Interventionen (Report Versorgungsforschung). Band 2; Deutscher Ärzteverlag, Köln 2010, 1-6

Anliker M, Beyeler L, Kissling A (2005): Erwartungen der Alters-und Pflegeheime an die Zusammenarbeit mit den Hausärztinnen und Hausärzten. PrimaryCare $\underline{5}, 647-649$

Arai H, Ouchi Y, Yokode M, Ito H, Uematsu H, Eto F, Oshima S, Ota K, Saito Y, Sasaki H (2012): Toward the realization of a better aged society: Messages from gerontology and geriatrics: Realization of a better aged society. Geriatr Gerontol Int 12, 16-22

Arnetz BB (2001): Psychosocial challenges facing physicians of today. Soc Sci Med $1982 \underline{52}, 203-213$

Bartholomeyczik S, Halek M: Pflege von Menschen mit Demenz. In: Jacobs K, Kuhlmey A, Greß S, Schwinger A, Klauber J (Hrsg.): Pflege-Report 2017. Schwerpunkt: Die Versorgung der Pflegebedürftigen; Schattauer, Stuttgart 2017, 51-59

Behmann M, Schmiemann G, Lingner H, Kühne F, Hummers-Pradier E, Schneider N (2012): Berufszufriedenheit von Hausärzten. Ergebnisse einer Befragung. Dtsch Ärztebl 11, 193-200

Blumenthal D, Gokhale M, Campbell EG, Weissman JS (2001): Preparedness for clinical practice: reports of graduating residents at academic health centers. JAMA $\underline{286}, 1027-1034$

Blüher S, Schnitzer S, Kuhlmey A: Der Zustand Pflegebedürttigkeit und seine Einflussfaktoren im hohen Lebensalter. In: Jacobs K, Kuhlmey A, Greß S, 
Schwinger A, Klauber J (Hrsg.): Pflege-Report 2017. Schwerpunkt: Die Versorgung der Pflegebedürftigen; Schattauer, Stuttgart 2017, 3-9

Bolmsjö BB, Strandberg EL, Midlöv P, Brorsson A (2015): "It is meaningful; I feel that I can make a difference" - A qualitative study about GPs' experiences of work at nursing homes in Sweden. BMC Fam Pract $\underline{16}, 111-119$

Buddeberg-Fischer B, Stamm M, Marty F (2007): Attraktivitätssteigerung der Hausarztmedizin - Ansichten und Vorschläge von praktizierenden Hausärzten. PrimaryCare $\underline{7}, 639-641$

Carrier M, Warschau G (2012): Warten, bis der Arzt kommt? Altenheim 7 , 3437

Corroon-Sweeney E, Murphy C, Collins DR (2009): Caring for Nursing Home Patients - A Primary Care Perspective. Ir Med J 102, 317-320

Erler A, Beyer M, Gerlach FM (2010): Ein Zukunftskonzept für die hausärztliche Versorgung in Deutschland. 2. Das Modell der Primärversorgungspraxis. Eine Darstellung anhand der Vorschläge des Sachverständigenrats Gesundheit 2009. ZFA Online $\underline{86}, 159-165$

Fleischmann N, Tetzlaff B, Werle J, Geister C, Scherer M, Weyerer S, Hummers-Pradier E, Mueller CA (2016): Interprofessional collaboration in nursing homes (interprof): a grounded theory study of general practitioner experiences and strategies to perform nursing home visits. BMC Fam Pract 17, 123-135

Flick U: Qualitative Sozialforschung: Eine Einführung. 3. Auflage; Rowohlt Taschenbuch, Reinbek bei Hamburg 2010

Gleeson LE, Jennings S, Gavin R, McConaghy D, Collins DR (2014): Primary care in nursing homes revisited: survey of the experiences of primary care physicians. Ir Med J $\underline{107}$, 234-236

Goetz K, Campbell SM, Steinhaeuser J, Broge B, Willms S, Szecsenyi J (2011): Evaluation of job satisfaction of practice staff and general practitioners: an exploratory study. BMC Fam Pract 12, 137-142 
Gothe H: Arbeits- und Berufszufriedenheit von Ärzten: Eine Übersicht der internationalen Literatur. In: Schwartz FW, Angerer P (Hrsg.): Arbeitsbedingungen und Befinden von Ärztinnen und Ärzten: Befunde und Interventionen (Report Versorgungsforschung). Band 2; Deutscher Ärzteverlag, Köln 2010, 7-14

Groom L, Avery AJ, Boot D, O'Neill C, Thornhill K, Brown K, Jones R (2000): The impact of nursing home patients on general practitioners' workload. $\mathrm{Br} \mathrm{J}$ Gen Pract $\underline{50}$, 473-476

Hallauer J, Bienstein C, Lehr U, Rönsch H: SÄVIP - Studie zur ärztlichen Versorgung in Pflegeheimen. Vincentz Network, Hannover 2005

Hansson A, Gunnarsson R, Mattsson B (2007): Balancing - an equilibrium act between different positions: An exploratory study on general practitioners' comprehension of their professional role. Scand J Prim Health Care 2드, 80-85

Hibbeler B (2007): Ärztliche Versorgung im Pflegeheim: Von Kooperation profitieren alle. Dtsch Arztebl 104, 3297-3300

Jacobs S (2003): Addressing the problems associated with general practitioners' workload in nursing and residential homes: findings from a qualitative study. Br J Gen Pract $\underline{53}, 113-119$

Kalitzkus V (2005): Die Bedeutung von Reflexivität und Positionierung im Prozess qualitativer Forschung in der Allgemeinmedizin. ZFA 81, 243-247

Karsch-Völk M, Lüssenheide J, Linde K, Schmid E, Schneider A (2015): Entwicklung eines Kriterienkatalogs für eine gelungene ärztliche Versorgung in Pflegeeinrichtungen. ZEFQ 109, 570-577

Katz PR, Karuza J, Intrator O, Mor V (2009): Nursing Home Physician Specialists: A Response to the Workforce Crisis in Long-Term Care. Ann Intern Med $\underline{150}, 411-413$

Kirsebom M, Wadensten B, Hedström M (2013): Communication and coordination during transition of older persons between nursing homes and hospital still in need of improvement. J Adv Nurs $\underline{69}, 886-895$ 
Kirsebom M, Hedström M, Pöder U, Wadensten B (2016): General practitioners' experiences as nursing home medical consultants. Scand J Caring Sci $\underline{31}$, $1-8$

Klose J, Uhlemann T, Gutschmidt S: Ärztemangel - Ärzteschwemme?: Auswirkungen der Altersstruktur von Ärzten auf die vertragsärztliche Versorgung. (WIdO-Materialien / Wissenschaftliches Institut der AOK. - Bonn: WIdO, 1996; 48); WIdO, Wiss. Inst. der AOK, Bonn 2003

Koch K, Miksch A, Schürmann C, Joos S, Sawicki PT (2011): The German Health Care System In International Comparison: The Primary Care Physicians' Perspective. Dtsch Ärztebl Int $\underline{108}, 255-261$

Krüger-Brand HE (2014): Ärztliche Pflegeheimbetreuung. Gut vernetzt geht es besser. Dtsch Arztebl Int 111, 24-25

Laag S, Müller T, Mruck M: Verantwortung gemeinsam tragen - die ärztliche Versorgung von Pflegeheimpatienten braucht eine Neuordnung. In: Barmer GEK Gesundheitswesen aktuell 2014 (Gesundheitswesen aktuell); Barmer GEK, Wuppertal 2014, 293-309

Lenander C, Midlöv P, Viberg N, Chalmers J, Rogers K, Bondesson A (2017): Use of Antipsychotic Drugs by Elderly Primary Care Patients and the Effects of Medication Reviews: A Cross-Sectional Study in Sweden. Drugs - Real World Outcomes $4,159-165$

Löffler C, Höck J, Hornung A, Kundt G, Drewelow E, Völker S, Kreiser B, Riedel J, Altiner A (2014): Was macht Ärzte glücklich? Berufszufriedenheit von Hausärzten in Mecklenburg-Vorpommern - eine repräsentative Querschnittsstudie. Gesundheitswesen 그, 927-931

Magin P, Catzikiris N, Tapley A, Morgan S, Holliday EG, Ball J, Henderson K, Elliott T, Regan C, Spike N (2017): Home visits and nursing home visits by early-career GPs: a cross-sectional study. Fam Pract $\underline{34}, 77-82$

Marx G, Wollny A (2009a): Qualitative Sozialforschung - Ausgangspunkte und Ansätze für eine forschende Allgemeinmedizin: Teil 1: Theorie und Grundlagen 
der qualitativen Forschung. ZFA $\underline{85}$, 105-113

Marx G, Wollny A (2009b): Qualitative Sozialforschung - Ausgangspunkte und Ansätze für eine forschende Allgemeinmedizin: Teil 2: Qualitative Inhaltsanalyse vs. Grounded Theory. ZFA $\underline{85}$, 467-476

McDermott C, Coppin R, Little P, Leydon G (2012): Hospital admissions from nursing homes: a qualitative study of GP decision making. Br J Gen Pract $\underline{62}$, 538-545

Meyer-Kühling I, Frankenberg C, Schröder J (2015): Erwartungshaltungen, Kommunikation und Kooperation von Pflegenden und Ärzten in der stationären Altenpflege. HBScience $\underline{6}, 70-75$

Müller CA, Tetzlaff B, Theile G, Fleischmann N, Cavazzini C, Geister C, Scherer M, Weyerer S, van den Bussche H, Hummers-Pradier E (2015): Interprofessional collaboration and communication in nursing homes: a qualitative exploration of problems in medical care for nursing home residents - study protocol. J Adv Nurs $\underline{71}, 451-457$

Natanzon I, Ose D, Szecsenyi J, Joos S (2010): Berufsperspektive Allgemeinmedizin: Welche Faktoren beeinflussen die Nachwuchsgewinnung? DMW Dtsch Med Wochenschr 135, 1011-1015

Nowossadeck E (2012): Demografische Alterung und Folgen für das Gesundheitswesen. Robert Koch-Institut Berlin (Hrsg.). GBE kompakt $\underline{3}$ (2), 1-8

Oosterveld-Vlug MG, Pasman HRW, van Gennip IE, Willems DL, OnwuteakaPhilipsen BD (2013): Nursing home staff's views on residents' dignity: a qualitative interview study. BMC Health Serv Res $\underline{13}$, 353-361

Pell J, Williams S (1999): Do nursing home residents make greater demands on GPs? A prospective comparative study. Br J Gen Pract $\underline{49}, 527-530$

Przyborski A, Wohlrab-Sahr M: Qualitative Sozialforschung: ein Arbeitsbuch (Lehr- und Handbücher der Soziologie). 3. Auflage.; Oldenbourg, München 2010 
Riedl M, Mantovan F, Them C (2013): Being a Nursing Home Resident: A Challenge to One's Identity. Nurs Res Pract 2013, 1-9

Schäfer H-M, Krentz H, Harloff R (2005): Berufszufriedenheit von Allgemeinärzten in Deutschland und Frankreich - eine vergleichende Untersuchung in 3

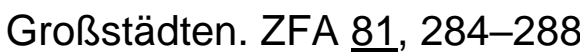

Schäfer H-M, Becker A, Krentz H, Reisinger E (2008): Wie zufrieden sind Hausärzte im Nordosten Deutschlands mit ihrem Beruf? - Ein Survey zur Berufszufriedenheit von Allgemeinärzten in Mecklenburg-Vorpommern. ZEFQ 102, $113-116$

Schlitt R (2009): Ärztliche Betreuung in Berliner Pflegeheimen. KV-Umfrage deckt Stärken und Mängel der Zusammenarbeit auf. KV Blatt $\underline{7}$, 28-29

Schneekloth U: Hilfe- und Pflegebedürftige in Alteneinrichtungen 2005.

Schnellbericht zur Repräsentativerhebung im Forschungsprojekt „Möglichkeiten und Grenzen selbständiger Lebensführung in Einrichtungen“ (MuG IV). Bundesministerium für Familie, Senioren, Frauen und Jugend, München 2006

Schröer N: Wissenssoziologische Hermeneutik. In: Hitzler R, Honer A (Hrsg.): Sozialwissenschaftliche Hermeneutik. Eine Einführung. Springer Fachmedien, Wiesbaden 1997, 109-129

Sharp R (2011): Vertrag verbessert Pflegeheim-Versorgung. Hausarzt 137, 20 21

Strauss A, Corbin J: Grounded Theory: Grundlagen Qualitativer Sozialforschung. Beltz, Weinheim 2010

Theile G, Kruschinski C, Buck M, Müller CA, Hummers-Pradier E (2011): Home visits - central to primary care, tradition or an obligation? A qualitative study. BMC Fam Pract 12, 24-35

Thürmann PA: Einsatz von Psychopharmaka bei Pflegebedürftigen. In: Jacobs K, Kuhlmey A, Greß S, Schwinger A, Klauber J (Hrsg.): Pflege-Report 2017. Schwerpunkt: Die Versorgung der Pflegebedürftigen; Schattauer, Stuttgart 
Tsakitzidis G, Anthierens S, Timmermans O, Truijen S, Meulemans H, Van Royen P (2017): Do not confuse multidisciplinary task management in nursing homes with interprofessional care! Prim Health Care Res Dev $\underline{18}$, 591-602

van den Bussche H, Weyerer S, Schäufele M, Lübke N, Schröfel S-C, Dietsche $S$ (2009): Die ärztliche Versorgung von Pflegeheimbewohnern in Deutschland eine kritische Würdigung der vorliegenden Studien: Medical care in nursing homes in Germany - a critical review of actual studies. ZFA $\underline{85}, 240-246$

van den Bussche $\mathrm{H}$ : Arbeitsbelastung und Berufszufriedenheit bei niedergelassenen Ärztinnen und Ärzten: Genug Zeit für die Patientenversorgung? In:

Schwartz FW, Angerer P (Hrsg.): Arbeitsbedingungen und Befinden von Ärztinnen und Ärzten: Befunde und Interventionen (Report Versorgungsforschung).

Band 2; Deutscher Ärzteverlag, Köln 2010, 235-242

van den Hombergh P, Künzi B, Elwyn G, van Doremalen J, Akkermans R, Grol R, Wensing M (2009): High workload and job stress are associated with lower practice performance in general practice: an observational study in 239 general practices in the Netherlands. BMC Health Serv Res $\underline{9}, 118-125$

Voltmer E, Rosta J, Siegrist J, Aasland OG (2012): Job stress and job satisfaction of physicians in private practice: comparison of German and Norwegian physicians. Int Arch Occup Environ Health 포, 819-828

Williams El, Savage S, McDonald P, Groom L (1992): Residents of private nursing homes and their care. Br J Gen Pract $\underline{42}$, 477-481

Wilm S (2005): Mitten in der Wirklichkeit und reflektiert. Neues Potenzial und neuer Ort für die allgemeinmedizinische Forschung. ZFA 81, 234-236

Wilm S, in der Schmitten J (2007): Was ist der Kern der hausärztlichen Tätigkeit? Prim Care $\underline{7}, 481-485$ 


\subsection{Internetquellen}

Bundesministerium der Justiz und für Verbraucherschutz (2017): SGB V, http://www.gesetze-im-internet.de/sgb_5/; Zugriff am 12.11.2017

Bundesministerium der Justiz und Verbraucherschutz (2017): SGB XI, § 71 Pflegeeinrichtungen, Abs. 2.

https://www.gesetze-im-internet.de/sgb_11/BJNR101500994.html; Zugriff am 12.11.2017

Bundesministerium für Gesundheit (2012): Das Pflege Neuausrichtungs Gesetz: Stand nach der 3. Lesung im Bundestag.

https://www.bundesgesundheitsministerium.de/fileadmin/dateien/Publikationen/ Pflege/Broschueren/Broschuere_Das_Pflege-NeuausrichtungsGesetz_Stand_nach_der_3._Lesung_im_Bundestag.pdf; Zugriff am 29.03.2015

DeFrances CJ, Hall MJ (2007): 2005 National Hospital Discharge Survey. Advance Data from Vital and Health Statistics; no 385. U.S. Department of Health and Human Services. Centers for Disease Control and Prevention. National Center for Health Statistics https://www.cdc.gov/nchs/data/ad/ad385.pdf; Zugriff am 31.01.2018

Deutscher Bundestag (2009): Sondergutachten 2009 des Sachverständigenrates zur Begutachtung der Entwicklung im Gesundheitswesen: Koordination und Integration - Gesundheitsversorgung in einer Gesellschaft des längeren Lebens. Deutscher Bundestag 2009. Drucksache 16/13770 http://dip21.bundestag.de/dip21/btd/16/137/1613770.pdf; Zugriff am 31.01.2018

Deutscher Bundestag (2012): Entwurf eines Gesetzes zur Neuausrichtung der Pflegeversicherung (Pflege-Neuausrichtungsgesetz - PNG).

Drucksache 17/9369 http://dip21.bundestag.de/dip21/btd/17/093/1709369.pdf; Zugriff am 31.01.2018

Deutscher Bundestag (2015): Gesetz zur Stärkung der Versorgung in der gesetzlichen Krankenversicherung (GKV-Versorgungsstärkungsgesetz - GKVVSG). Bundesgesetzblatt Jahrgang 2015 Teil I Nr. 30, ausgegeben zu Bonn am 22. Juli 2015 
https://www.bgbl.de/xaver/bgbl/start.xav?startbk=Bundesanzeiger_BGBI\&jump To=bgbl115s1211.pdf\#_bgbl_\%2F\%2F*5B\%40attr_id\%3D\%27bgbl115s12 11.pdf\%27\%5D_1513024419772; Zugriff am 31.01.2018

Kalitzkus V (2011): ZuVerSicht: Die Zukunft der hausärztlichen Versorgung aus gesundheitsberuflicher und Patienten-Sicht. Eine qualitative Lokalstudie in zwei ländlichen Versorgungsregionen Nordrhein-Westfalens. Witten 2011 https://www.uni-wh.de/fileadmin/media/g/medi/g_med_i_allgemeinmedizin/Forschung/Projekte/ZuVerSicht_Projektbericht_7April2011.pdf; Zugriff am 03.07.2016

Kassenärztliche Bundesvereinigung (2016): Praxisnachrichten - Pflegeheimversorgung: Die neuen Leistungen im Überblick. http://www.kbv.de/html/1150_23356.php; Zugriff am 14.12.2017

Kassenärztliche Bundesvereinigung (2018): Einheitlicher Bewertungsmaßstab (EBM). Stand: 1. Quartal 2018. Arztgruppen-EBM Hausarzt. Berlin 2018 http://www.kbv.de/media/sp/EBM_Hausarzt_20180101_V1.pdf; Zugriff am 01.02 .2018

Kassenärztliche Vereinigung Niedersachsen (2017): Arzneimittelvereinbarung gemäß § 84 Abs. 1 SGB V über die Versorgungs- und Wirtschaftlichkeitsziele sowie Kriterien für Sofortmaßnahmen zur Einhaltung des Ausgabenvolumens der Arznei- und Verbandmittel für das Jahr 2017.

http://www.kvn.de/icc/c/ueber-uns/amtliche-

bekanntmachungen/binarywriterservlet?imgUid=9d55e60a-d9d9-e513-68a3c220b8ff6bcb\&uBasVariant=11111111-1111-1111-1111-111111111111; Zugriff am 31.01.2018

Kassenärztliche Vereinigung Niedersachsen (2018): Aufstellung der KVinternen Gebührenordnungspositionen in Niedersachsen 1. Quartal 2018. http://www.kvn.de/icc/c/Praxis/Abrechnung/KVN-interneGebuehrenordnungspositionen/binarywriterservlet?imgUid=e2210974-fdddf513-68a3-c220b8ff6bcb\&uBasVariant=11111111-1111-1111-1111-

111111111111; Zugriff am 01.02.2018 
MAXQDA. http://www.maxqda.de/; Zugriff am 30.03.2015

Mühlmeyer-Mentzel A, Schürmann I (2011): Softwareintegrierte Lehre der Grounded-Theory-Methodologie. Forum Qualitative Sozialforschung / Forum:

Qualitative Social Research $\underline{12}$

http://www.qualitative-research.net/index.php/fqs/article/view/1654/3266\#gaut;

Zugriff am 14.12.2017

Niehus H, Berger B, Stamer M, Schmacke N (2008): „Die Sicherung der hausärztlichen Versorgung in der Perspektive des ärztlichen Nachwuchses und niedergelassener Hausärztinnen und Hausärzte“. Bremen 2008 https://www.researchgate.net/profile/Bettina_Berger/publication/236199133_Die _Sicherung_der_hausarztlichen_Versorgung_in_der_Perspektive_des_arztlich en_Nachwuchses_und_niedergelassener_Hausarztinnen_und_Hausarzte_Abs chlussbericht/links/00b49516eb195618cd000000/Die-Sicherung-derhausaerztlichen-Versorgung-in-der-Perspektive-des-aerztlichen-Nachwuchsesund-niedergelassener-Hausaerztinnen-und-HausaerzteAbschlussbericht.pdf?origin=publication_list; Zugriff am 31.01.2018

Statistisches Bundesamt (2009): Bevölkerung Deutschlands bis 2060. 12. koordinierte Bevölkerungsvorausberechnung. Wiesbaden 2009 https://www.destatis.de/DE/Publikationen/Thematisch/Bevoelkerung/Vorausber echnungBevoelke-

rung/BevoelkerungDeutschland2060Presse5124204099004.pdf?_blob=public ationFile; Zugriff am 31.01.2018

Statistisches Bundesamt (2017): Pflegestatistik 2015 - Pflege im Rahmen der Pflegeversicherung - Deutschlandergebnisse. Wiesbaden 2017 https://www.destatis.de/DE/Publikationen/Thematisch/Gesundheit/Pflege/Pflege Deutschlandergebnisse5224001159004.pdf?_blob=publicationFile; Zugriff am 31.01 .2018 


\section{Danksagung}

Mein Dank gilt Frau Prof. Dr. med. E. Hummers, Direktorin des Instituts für Allgemeinmedizin an der Georg-August-Universität Göttingen, für die Ermöglichung dieser Promotion, die freundliche Überlassung des Promotionsthemas sowie die konstruktive Unterstützung und fördernde Betreuung.

Zudem möchte ich mich herzlich bei Frau Dr. med. C. Müller für ihr zeitintensives Engagement und die vielen wertvollen und produktiven Hilfestellungen bei der Erstellung dieser Dissertation bedanken.

Des Weiteren möchte ich meinen Dank an die Teammitglieder der INTERPROF-Studie richten, mit denen ich während meiner Promotionszeit zusammenarbeiten durfte, ebenso wie an die ärztlichen Kollegen für ihre Teilnahme an der Studie. 